\title{
CENTRAL SCHEMES FOR THE MODIFIED BUCKLEY-LEVERETT EQUATION
}

\author{
DISSERTATION
}

Presented in Partial Fulfillment of the Requirements for the Degree Doctor of Philosophy in the Graduate

School of the Ohio State University

By

Ying Wang, B.Sc., M.Sc., M.A.S.

Graduate Program in Mathematics

The Ohio State University

2010

\section{Dissertation Committee:}

Professor Chiu-Yen Kao, Advisor

Professor Avner Friedman

Professor Fei-Ran Tian 
(c) Copyright by

Ying Wang

July, 2010 


\begin{abstract}
In fluid dynamics, the Buckley-Leverett (BL) equation is a transport equation used to model two-phase flow in porous media. One application is secondary recovery by water-drive in oil reservoir simulation. The modified Buckley-Leverett (MBL) equation differs from the classical BL equation by including a balanced diffusivedispersive combination. The dispersive term is a third order mixed derivatives term, which models the dynamic effects in the pressure difference between the two phases. The classical BL equation gives a monotone water saturation profile for any Riemann problem; on the contrast, when the dispersive parameter is large enough, the MBL equation delivers non-monotone water saturation profile for certain Riemann problems as suggested by the experimental observations.

In this thesis, we first show that the solution of the finite interval $[0, L]$ boundary value problem converges to that of the half-line $[0,+\infty)$ problem for the MBL equation as $L \rightarrow+\infty$. This result provides a justification for the use of the finite interval boundary value problem in numerical studies for the half line problem.

Furthermore, we extend the classical central schemes for the hyperbolic conservation laws to solve the MBL equation which is of pseudo-parabolic type. This extension can also be applied to other conservation law solvers. Numerical results confirm
\end{abstract}


the existence of non-monotone water saturation profiles consisting of constant states separated by shocks, which is consistent to the experimental observations.

The two-dimensional physical space is a general setting for the underground oil recovery. In this thesis, we also include the derivation of the two-dimensional extension of the MBL equation. 
To my teachers 


\section{ACKNOWLEDGMENTS}

I would first like to address special thanks to my advisor, Prof. Chiu-Yen Kao for her generous support, constant advice and endless patience in improving the results of this work, and more importantly, for setting a role model as a dedicating woman mathematician.

I also want to thank my committee members, Profs. Avner Friedman and Fei-Ran Tian for their guidance and encouragements, not only during this thesis preparation process, but also throughout the years I stayed at OSU.

It has been a long journey since the time I left home. What is happening now all started from an undergraduate scholarship I was awarded by Singapore Ministry of Education, without which, I would not have gone this far. It were the many Computer Scientists and Mathematicians I met at National University of Singapore (NUS) who led me into the beautiful science world, among which, the lecture clips and conversation moments with Profs. Tay Yong Chiang and Andrew Lim still flash through my mind every now and then. Meanwhile then, the professors and graduate students I met at computational science department, NUS, motivated most of my early research work. This joint effort has later benefited my entire graduate school studies.

I switched major of study from Computer Science to Mathematics when I started 
graduate school study at Georgia Tech. Amazingly enough, the Mathematics professors at Georgia Tech transformed me from one who could barely work out a calculus problem to a qualified Mathematics Ph.D. student.

In addition to the Georgia Tech professors, I want to express my gratitude to Profs. Douglas Ulmer at University of Arizona then and Weishi Liu at University of Kansas, who invited me for campus visits when I applied for the Ph.D. programs in their institutions. Their approval and recognition were more encouraging to me than they may have thought. Along the same line, I would like to thank Prof. Saleh Tanveer for having me here as a Mathematics Ph.D. student.

I have benefited from the tutelage of several faculty and staff here at the Ohio State University. In particular, my appreciation goes to my recommendation letter writers. Thank you all for paving the way for my future development in academia.

It is my deepest desire to dedicate this work to all my teachers, at every stage of my life.

I am also indebted to all my friends. The friends I met in Singapore have painted my college life as colorful as it could be. Some are still very influential to date. The midnight pendulum conversation, the movie premieres, the Pandan valley days, and many more instances remind me the happy time.

The friends I met in the United States have been good companion. They are the sweetest memory of my graduate school experience. Especially, the honest criticisms have always helped me re-orient myself away from the myriad distractive matters.

To all my friends, younger and older, local and remote, men and women, I am 
deeply grateful to your support for all these years. The joys and tears we shared shaped my life to the full extent, both personally and academically.

Last but not least, I want to thank my parents, for many reasons. The freedom they provided to me when I was little gave me infinite opportunities to explore the world; the unconditional love they constantly express to me held me through the rough days. I wish them well. 


\section{VITA}

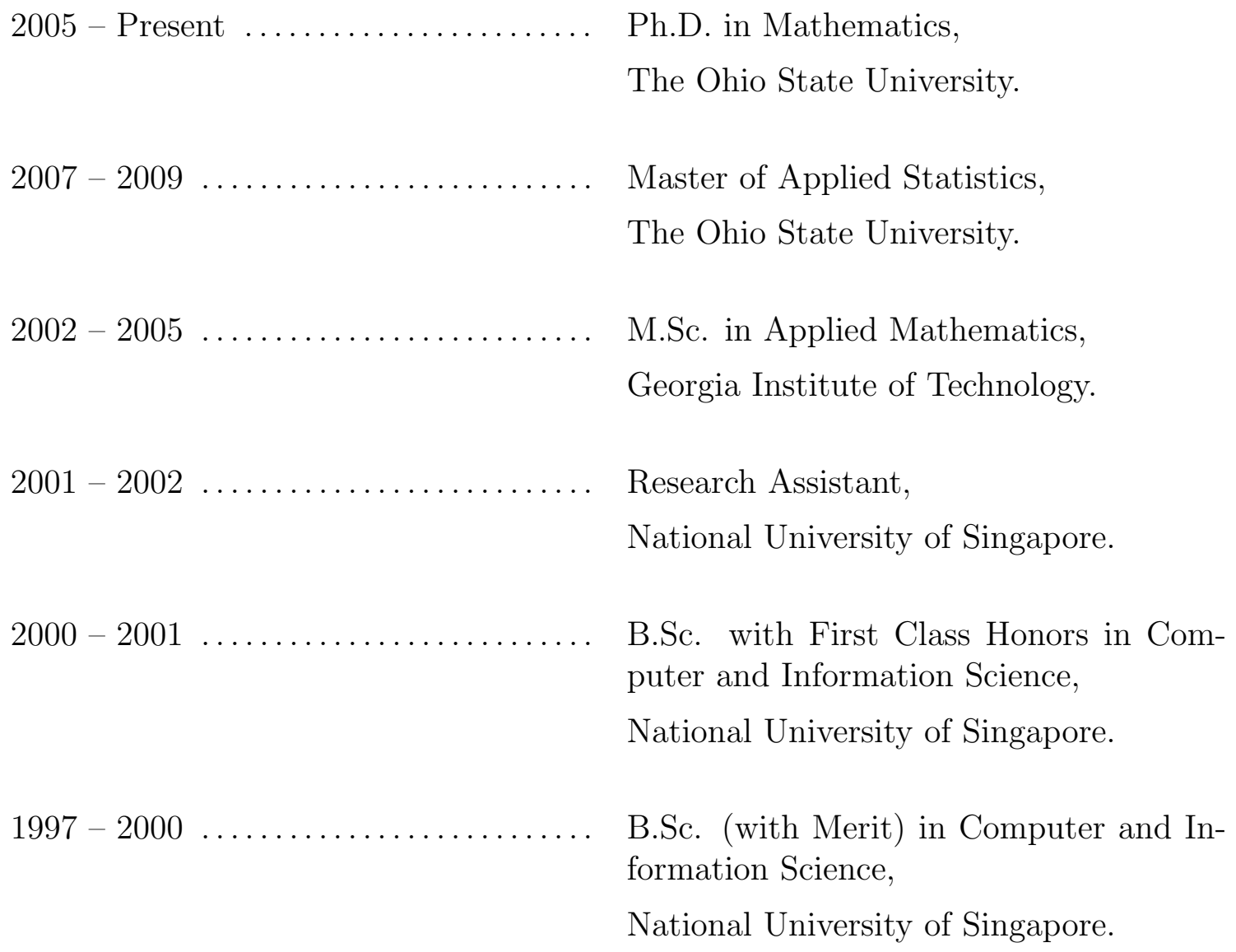




\section{FIELDS OF STUDY}

Major Field: Applied Mathematics

Studies in Numerical Solutions of Hyperbolic Conservation Laws Numerical Analysis and Scientific Computing

Application of Differential Equations 


\section{TABLE OF CONTENTS}

Abstract.................................... ii

Dedication . . . . . . . . . . . . . . . . . . iv

Acknowledgments . . . . . . . . . . . . . . . . . . . . v v

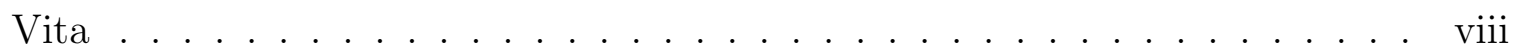

List of Figures . . . . . . . . . . . . . . . . . . xii

List of Tables . . . . . . . . . . . . . . . . . . . . xiv

CHAPTER PAGE

$1 \quad$ Introduction . . . . . . . . . . . . . . . . . . . . . . 1

$1.1 \quad$ Underground Oil Production Process $\ldots \ldots \ldots \ldots$

$1.2 \quad$ Classical Buckley-Leverett (BL) Equation . . . . . . . . . 4

1.3 Derivation of the Modified Buckley-Leverett Equation . . 11

$2 \quad$ Half Line v.s. Finite Interval Domain _... . . . . . . . . . . . 19

$2.1 \quad$ Half Line Problem . . . . . . . . . . . . . . . . . . . . 20

$2.2 \quad$ Finite Interval Boundary Value Problem . . . . . . . . 25

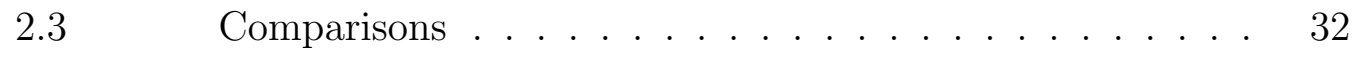

2.3 .1 Definitions and Lemmas . . . . . . . . . . . . . . . . . 33

$2.3 .2 \quad$ A Proposition . . . . . . . . . . . . . . . . . . . . 36

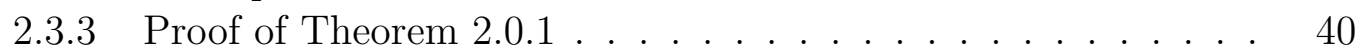

$3 \quad$ Numerical Schemes $\ldots \ldots \ldots$. . . . . . . . . . . . . . . 47

$3.1 \quad$ Second-Order Schemes _. . . . . . . . . . . . 47

3.1 .1 Trapezoid Scheme $\ldots \ldots \ldots$

$5.1 .2 \quad$ Midpoint Scheme $\ldots \ldots \ldots$. . . . . . . . . . . . . . 52 
$3.2 \quad$ A Third Order Semi-discrete Scheme. . . . . . . . . . 54

$4 \quad$ Computational Results $\ldots \ldots \ldots \ldots$

$4.1 \quad$ Accuracy Analysis $\ldots \ldots \ldots \ldots$

4.2 Traveling-Wave Solutions _. . . . . . . . . . . . . 62

$4.3 \quad$ Parameter Regimes $\ldots \ldots \ldots \ldots$

$4.4 \quad$ Examples . . . . . . . . . . . . . . . . . 67

$5 \quad$ 2D Modified Buckley-Leverett Equation $\ldots \ldots \ldots \ldots$

$5.1 \quad$ Derivation of the 2D MBL Equation . . . . . . . 80

6 Conclusion $\ldots \ldots \ldots \ldots \ldots \ldots$

\section{APPENDICES}

A Proof of the lemmas . . . . . . . . . . . . . . . 87

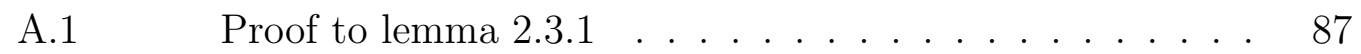

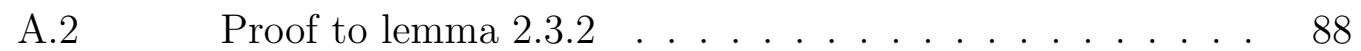

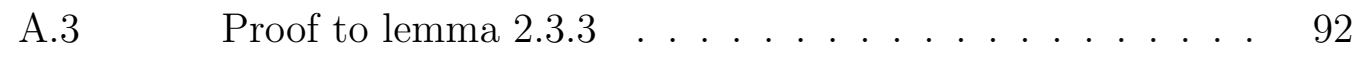

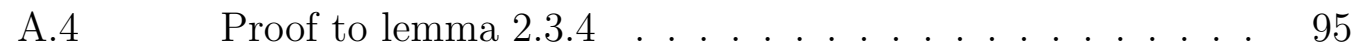

Bibliography . . . . . . . . . . . . . . . . . . . . . . . . 97 


\section{LIST OF FIGURES}

1.1 Demonstration of secondary recovery during the underground oil production process (courtesy to MPG Petroleum, Inc. http://mpgpetroleum . com/fundamentals.html)................ 3

1.2 The flux function and its derivative for Buckley-Leverett equation: (a)|| $f(u)=\frac{u^{2}}{u^{2}+M(1-u)^{2}} ; \quad$ (b) $\mid f^{\prime}(u)=\frac{2 M u(1-u)}{\left(u^{2}+M(1-u)^{2}\right)^{2}}$ with $M=2 . \ldots 5$

$1.3 \quad(\mathrm{a})||$ : The triple-valued solution of BL; (b)|: Determination of the shock location using the equal area rule. . . . . . . . . . . . . . . . 7

1.4 The entropy solution of the classical BL equation $\left(M=2, \alpha=\sqrt{\frac{2}{3}} \approx\right.$ 0.8165). (a) $0<u B=0.7 \leq \alpha$, the solution consists of one shock at $\frac{x}{t}=\frac{f\left(u_{B}\right)}{u_{B}} ;$ (b) $\alpha<u B=0.98<1$, the solution consists of a rarefaction between $u_{B}$ and $\alpha$ for $f^{\prime}\left(u_{B}\right)<\frac{x}{t}<f^{\prime}(\alpha)$ and a shock at $\frac{x}{t}=\frac{f(\alpha)}{\alpha}$. . . . . . . . . . . . . . . . . . . . 9

1.5 Courtesy to [8]: Snapshots of the saturation profile versus depth for six different applied fluxes in initially dry 20/30 sand measured using light transmission. At the highest $(11.8 \mathrm{~cm} / \mathrm{min})$ and lowest $\left(7.9 \times 10^{-4}\right.$ $\mathrm{cm} / \mathrm{min}$ ) fluxes the profiles are monotonic with distance and no saturation overshoot is observed, while all of the intermediate fluxes exhibit saturation overshoot. . . . . . . . . . . . . . . . . . . . 10

$4.1 \quad$ Critical values of $u$ when $M=2: \alpha \approx 0.816$ and $\beta \approx 1.147 . \quad$. . . . . 63

4.2 The bifurcation diagram of the MBL equation (4.1) with the bifurcation parameters $\left(\tau, u_{B}\right)$, where $M=2 . \ldots \ldots 66$ 
$4.3 \quad$ Given a fixed $\tau$, the three qualitatively different solution profiles due to different values of $u_{B}$. In particular, when $\tau>\tau_{*}$ and $\underline{u}<u_{B}<\bar{u}$, the solution profiles (Figure $4.3(\mathrm{~b})$ ) displays non-monotonicity, which is consistent with the experimental observations ([8]). Figures $4.3(\mathrm{a})]$ 4.3(b) and $4.3(\mathrm{c})$ are demonstrative figures. . . . . . . . . . . . 68

4.4 Numerical solutions to MBL equation with parameter settings fall in different regimes of the bifurcation diagram (Figure 4.2). The color coding is for different time: $\frac{1}{4} T$ (blue), $\frac{2}{4} T$ (green), $\frac{3}{4} T$ (magenta) and $T$ (black). The results are discussed in examples $1-6$. In figures $4.4(\mathrm{~d})|-| 4.4(\mathrm{f}), \alpha=\sqrt{\frac{M}{M+1}}=\sqrt{\frac{2}{3}}$ for $M=2 . \ldots \ldots 71$

4.5 The numerical solutions of the MBL equation at $\mathrm{T}=1$ with $\tau=0$ and different $u_{B}$ values. The results are discussed in example 7. . . 74

4.6 Numerical solutions to MBL equation with $u_{B}$ close to $\bar{u}_{\tau=5} \approx 0.98$. The color coding is for different time: $\frac{1}{4} T$ (blue), $\frac{2}{4} T$ (green), $\frac{3}{4} T$ (magenta) and $T$ (black). The results are discussed in example 8. . . 75

4.7 Numerical solutions to MBL equation with $u_{B}$ close to $\underline{u}_{\tau=5} \approx 0.68$. The color coding is for different time: $\frac{1}{4} T$ (blue), $\frac{2}{4} T$ (green), $\frac{3}{4} T$ (magenta) and $T$ (black). The results are discussed in example 9. . . 76

4.8 Numerical solutions to MBL equation with small constant $u_{B}=0.6$ and different $\tau$ values. The figures on the second and third rows are the magnified versions of the first row at $t=\frac{1}{4} T$ and $t=T$ respectively. The color coding is for different time: $\frac{1}{4} T$ (blue), $\frac{2}{4} T$ (green), $\frac{3}{4} T$ \begin{tabular}{ll|l} 
(magenta) and $T$ (black). The results are discussed in examples 10. & 78
\end{tabular}

4.9 The numerical solutions of MBL equation at $T=0.5$ with $\epsilon=0.001$ (blue), $\epsilon=0.002$ (yellow), $\epsilon=0.003$ (magenta), $\epsilon=0.004$ (green), and $\epsilon=0.005$ (black). The view windows are zoomed into the regions where different $\epsilon$ values impose different solution profiles. The results are discussed in example 11. . . . . . . . . . . . . . . . . . 79 


\section{LIST OF TABLES}

TABLE

PAGE

$3.1 \quad$ Flow chart for Trapezoid Scheme . . . . . . . . . . . . . . . . . . 51

$3.2 \quad$ Flow chart for Midpoint Scheme . . . . . . . . . . . . . . . . . . . 53

4.1 The accuracy test for the trapezoid scheme for the MBL equation (4.1) with $\epsilon=1$ and $M=2$. . . . . . . . . . . . . . . . . . . 60

4.2 The accuracy test for the third order semi-discrete scheme for the MBL equation (4.1) with $\epsilon=1$ and $M=2$. . . . . . . . . . . . . . . . . . 61

4.39 pairs of $\left(\tau, u_{B}\right)$ values with either fixed $\tau$ value varying $u_{B}$ values or fixed $u_{B}$ value varying $\tau$ values used in Examples $1-6$. Notice that

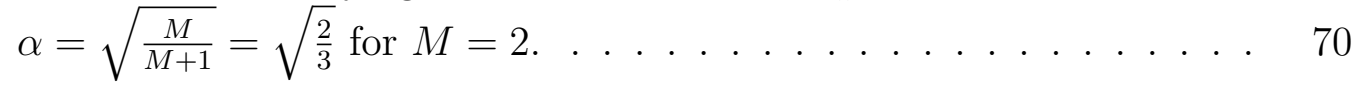




\section{CHAPTER 1 INTRODUCTION}

For the past fifth years, various research activities have been contacted to assist oilreservoir management. The main purpose is to provide an information database that can help the oil companies maximize the oil and gas recovery. Unfortunately, to obtain an accurate prediction of reservoir flow scenarios is a challenging task. One of the reasons is that we can never get a complete and concrete characterization of the rock parameters that influence the flow pattern. And even if we did, we would not be able to model the process that utilizes all available information, since this would require a tremendous amount of computer resources that exceed by far the capabilities of modern multi-processor computers. On the other hand, we do not need, nor do we seek a simultaneous description of the flow scenario on all scales down to the pore scale. For reservoir management it is usually sufficient to provide the general trends in the reservoir flow pattern.

In this chapter, we will first provide some background knowledge of the underground oil production process in section 1.1. In section 1.2, we will then introduce the classical Buckley-Leverett (BL) equation, which models the water-saturation during secondary recovery by water-drive in oil reservoir simulation. Despite the simplicity, BL equation has its limitations. To improve the model in the sense of getting a 
saturation profile which is more consist to the experimental observations, we consider the modified Buckley-Leverett (MBL) equation. In section 1.3, we will show the derivation of MBL equation.

\subsection{Underground Oil Production Process}

Initially, an oil reservoir is at an equilibrium stage, and contains oil/gas, and water, separated by gravity. This equilibrium has been established over millions of years with gravitational separation and geological and geothermal processes. When a well is drilled through the upper non-permeable layer and penetrates the upper oil cap, this equilibrium is immediately disturbed. Oil flows out of the reservoir due to overpressure. This in turn, sets up a flow inside the reservoir and oil flows towards the well, which in turn may induce gravitational instabilities. Also the capillary pressures will act as a (minor) driving mechanism, resulting in local perturbations of the situation. During the above process, perhaps 20 percent of the oil present is produced until a new equilibrium is achieved. This process is called primary production by natural drives. Notice that a sudden drop in pressure also may have numerous other intrinsic effects.

As pressure drops, less oil/gas is flowing, and eventually the production is no longer economically sustainable. Then the operating company may start secondary production by engineered drives. These are processes based on injecting water or gas into the reservoir (see Figure 1.1). The reason for doing this is two folds: some of the pressure is rebuilt or even increased, and secondly one tries to push out more profitable oil 


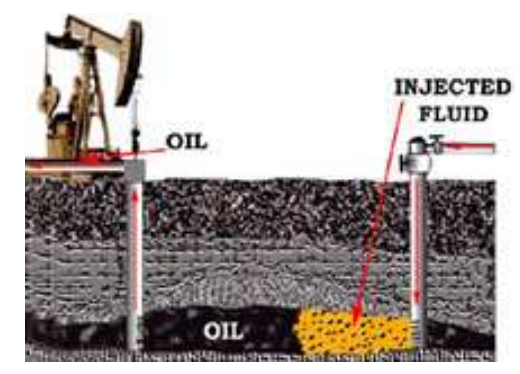

Figure 1.1: Demonstration of secondary recovery during the underground oil production process (courtesy to MPG Petroleum, Inc. http://mpgpetroleum. com/fundamentals.html).

with the injected substance. One may perhaps produce another 20 percent of the oil by such processes and engineered drives are standard procedure at most locations. This process is called secondary recovery.

In order to produce even more oil, Enhanced Oil Recovery (EOR, or tertiary recovery) techniques may be employed. Among these are heating the reservoir or injection of sophisticated substances like foam, polymers or solvents. Polymers are supposed to change the flow properties of water, and thereby to more efficiently push out oil. Similarly, solvents change the flow properties of the oil, for instance by developing miscibility with an injected gas. In some sense, one tries to wash the pore walls for most of the remaining oil. The other technique is based on injecting steam, which will heat the rock matrix, and thereby, hopefully, change the flow properties of the oil. At present, such EOR techniques are considered too expensive for large-scale commercial 
use, but several studies have been conducted and the mathematical foundations are being carefully investigated, and at smaller scales EOR is being performed.

Here, the terms primary, secondary, and tertiary are ambiguous. EOR techniques may be applied during primary production, and secondary recovery may be performed from the first day of production.

In this thesis, we focus on the mathematical modeling and numerical simulations of the secondary recovery process. For the ease of modeling, we start with onedimensional model describing the horizontal flow.

\subsection{Classical Buckley-Leverett (BL) Equation}

The classical Buckley-Leverett (BL) equation [3] is a simple and effective model for two-phase fluid flow in a porous medium. One application is secondary recovery by water-drive in oil reservoir simulation. In this case, the two phases are oil and water, and the flow takes place in a porous medium of rock or sand. In one space dimension the equation has the standard conservation form

$$
\begin{aligned}
& u_{t}+(f(u))_{x}=0 \quad \text { in } \quad Q=\{(x, t): x>0, t>0\} \\
& u(x, 0)=0 \quad x \in(0, \infty) \\
& u(0, t)=u_{B} \quad t \in[0, \infty)
\end{aligned}
$$

with the flux function $f(u)$ being defined as

$$
f(u)= \begin{cases}0 & u<0, \\ \frac{u^{2}}{u^{2}+M(1-u)^{2}} & 0 \leq u \leq 1, \\ 1 & u>1 .\end{cases}
$$


In this content, $u: \bar{Q} \rightarrow[0,1]$ denotes the water saturation, and so lies between 0 and 1 (e.g. $u=1$ means pure water, and $u=0$ means pure oil), $u_{B}$ is a constant which indicates water saturation at $x=0$, and $M>0$ is a constant representing the water/oil viscosity ratio. The classical BL equation (1.1) is a prototype for conservation laws with convex-concave flux functions. The graph of $f(u)$ and $f^{\prime}(u)$ with $M=2$ is given in Figure 1.2. Notice that in Figure 1.2 (a), at $u=\alpha$, the tangent line coincide with the secant line connecting $(\alpha, f(\alpha))$ and $(0, f(0))$. We will discuss the meaning of this point in the later part of this section.

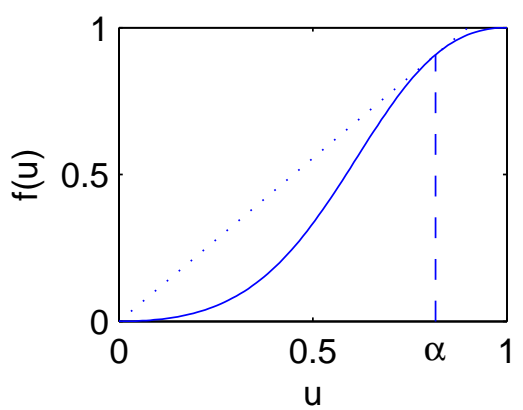

(a)

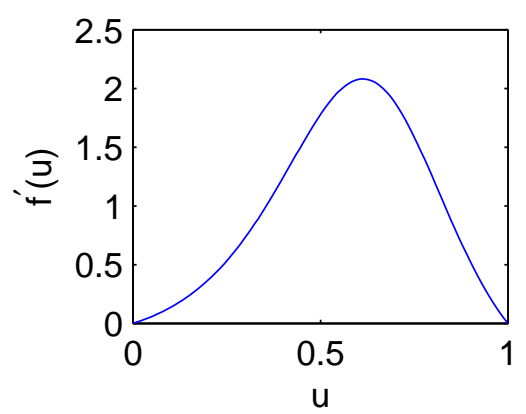

(b)

Figure 1.2: The flux function and its derivative for Buckley-Leverett equation: (a) $f(u)=\frac{u^{2}}{u^{2}+M(1-u)^{2}} ;$ (b) $f^{\prime}(u)=\frac{2 M u(1-u)}{\left(u^{2}+M(1-u)^{2}\right)^{2}}$ with $M=2$.

Due to the possibility of the existence of shocks in the solution of the hyperbolic 
conservation laws (1.1), the weak solutions are sought. The function $u \in L^{\infty}(Q)$ is called a weak solution of the conservation laws (1.1) if

$$
\int_{Q}\left\{u \frac{\partial \phi}{\partial t}+f(u) \frac{\partial \phi}{\partial x}\right\}=0 \quad \text { for all } \quad \phi \in C_{0}^{\infty}(Q) .
$$

Notice that the weak solution is not unique. Among the weak solutions, the entropy solution is physically relevant and unique. The weak solution that satisfies the Oleinik entropy condition [18]

$$
\frac{f(u)-f\left(u_{l}\right)}{u-u_{l}} \geq s \geq \frac{f(u)-f\left(u_{r}\right)}{u-u_{r}} \quad \text { for all } u \text { between } u_{l} \text { and } u_{r}
$$

is the entropy solution, where $u_{l}, u_{r}$ are the function values to the left and right of the shock respectively, and the shock speed $s$ satisfies the Rankine-Hugoniot jump condition [16, 11]

$$
s=\frac{f\left(u_{l}\right)-f\left(u_{r}\right)}{u_{l}-u_{r}}
$$

Consider the Riemann problem with initial condition

$$
u(x, 0)=\left\{\begin{array}{ll}
u_{B} & \text { if } x=0 \\
0 & \text { if } \quad x>0
\end{array},\right.
$$

by following the characteristics, we can construct the triple-valued solution as shown in Figure 1.3(a), Notice that the characteristic velocities are $f^{\prime}(u)$ so that the profile at time $t$ is simply the graph of $t f^{\prime}(u)$ turned sideways [14]. We can use the equal area rule:

the shaded area on the left of the shock $=$ the shaded area on the right of the shock 


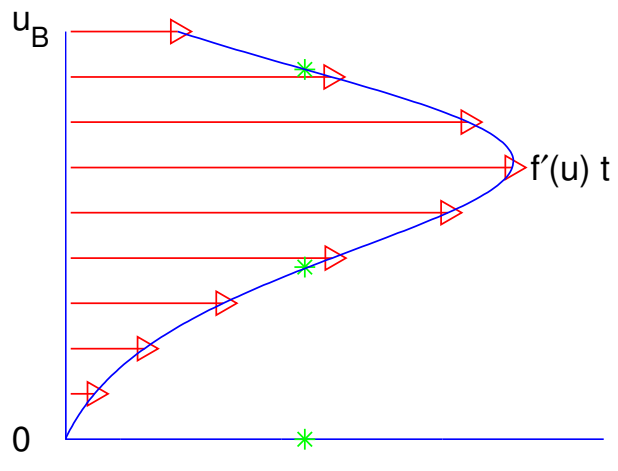

(a)

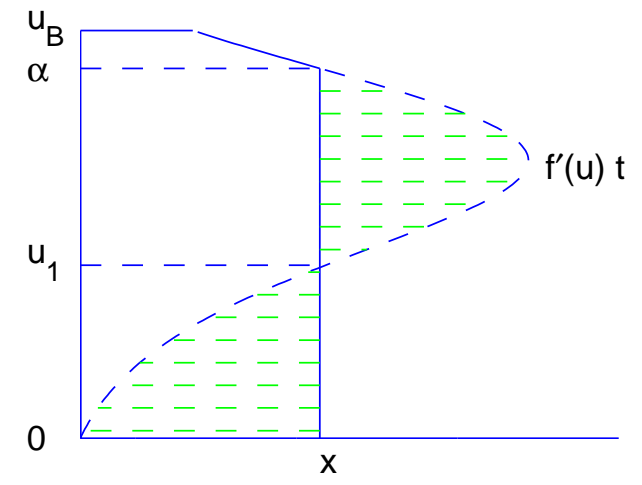

(b)

Figure 1.3: (a): The triple-valued solution of BL; (b): Determination of the shock location using the equal area rule.

as shown in Figure $1.3(\mathrm{~b})$, i.e.

$$
\begin{aligned}
\int_{u_{1}}^{\alpha} t f^{\prime}(u) d u-x\left(\alpha-u_{1}\right) & =x u_{1}-\int_{0}^{u_{1}} t f^{\prime}(u) d u \\
t\left(f(\alpha)-f\left(u_{1}\right)\right)-x \alpha & =-t\left(f\left(u_{1}\right)-f(0)\right) \\
x & =\frac{f(\alpha)}{\alpha} t
\end{aligned}
$$

Furthermore, from the triple-valued solution, we have that

$$
x=t f^{\prime}(\alpha)
$$

therefore, the post-shock value $\alpha$ satisfies

$$
f^{\prime}(\alpha)=\frac{f(\alpha)}{\alpha}
$$


which gives that

$$
\alpha=\sqrt{\frac{M}{M+1}} .
$$

The location of $\alpha$ and the special property (1.5) that $\alpha$ satisfies are shown in Figure 1.2 (a) for $M=2$.

Notice that in this case, $u_{r}=0$ and $f\left(u_{r}\right)=0$, hence the Rankine-Hugoniot condition (1.4) is automatically satisfies. Hence, replacing the triple-valued solution by a shock located at

$$
x=\frac{f(\alpha)}{\alpha} t
$$

gives the entropy solution. And the entropy solution of the classical BL equation can be classified into two categories:

1. If $0<u_{B} \leq \alpha$, the entropy solution has a single shock at $\frac{x}{t}=\frac{f\left(u_{B}\right)}{u_{B}}$.

2. If $\alpha<u_{B}<1$, the entropy solution contains a rarefaction between $u_{B}$ and $\alpha$ for $f^{\prime}\left(u_{B}\right)<\frac{x}{t}<f^{\prime}(\alpha)$ and a shock at $\frac{x}{t}=\frac{f(\alpha)}{\alpha}$.

These two types of solutions are shown in Figure 1.4 for $M=2$. In either case, the entropy solution of the classical BL equation (1.1) is a non-increasing function of $x$ at any given time $t>0$. However, the experiments [8] of two-phase flow in porous medium reveal complex infiltration profiles, which may involve overshoot, i.e., profiles may not be monotone as given in Figure 1.5. This suggests the need of modification to the classical BL equation (1.1). 


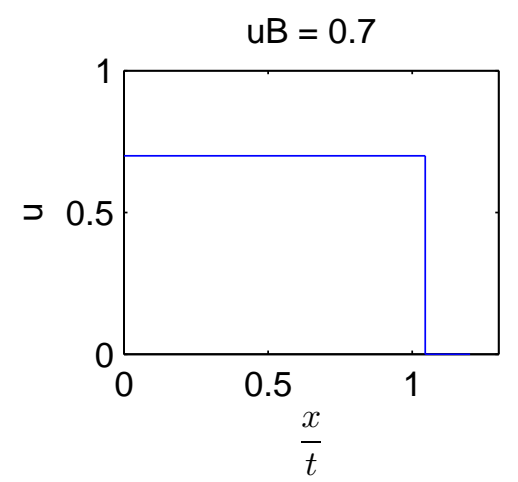

(a)

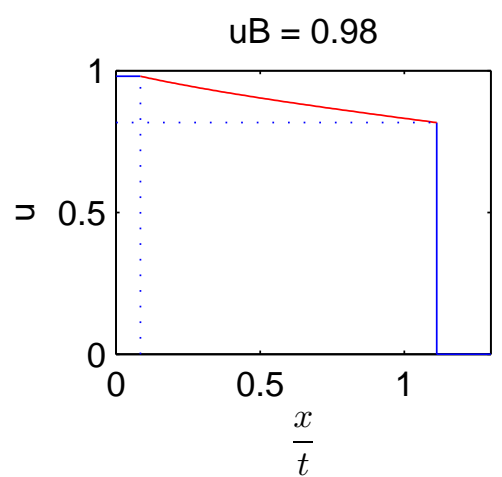

(b)

Figure 1.4: The entropy solution of the classical BL equation $\left(M=2, \alpha=\sqrt{\frac{2}{3}} \approx\right.$ 0.8165). (a) $0<u B=0.7 \leq \alpha$, the solution consists of one shock at $\frac{x}{t}=\frac{f\left(u_{B}\right)}{u_{B}} ;[(\mathrm{b})] \alpha<u B=0.98<1$, the solution consists of a rarefaction between $u_{B}$ and $\alpha$ for $f^{\prime}\left(u_{B}\right)<\frac{x}{t}<f^{\prime}(\alpha)$ and a shock at $\frac{x}{t}=\frac{f(\alpha)}{\alpha}$. 


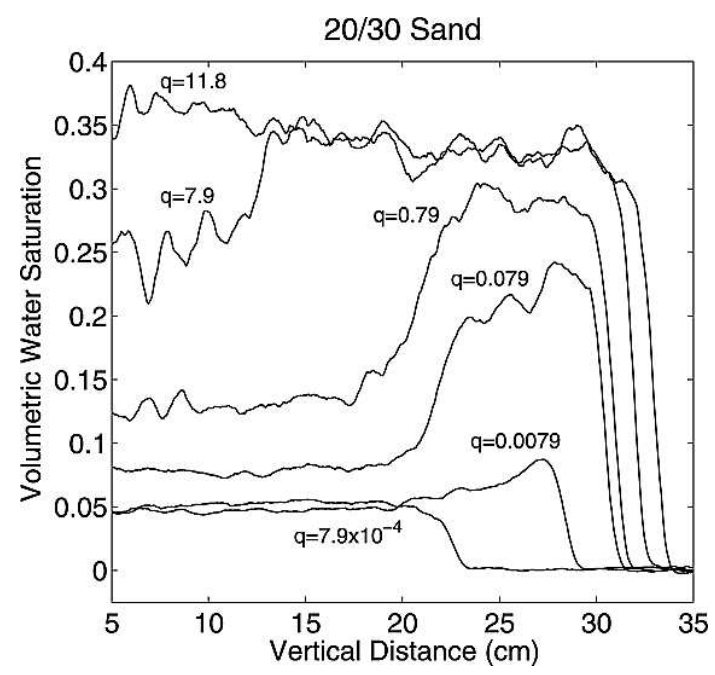

Figure 1.5: Courtesy to [8]: Snapshots of the saturation profile versus depth for six different applied fluxes in initially dry $20 / 30$ sand measured using light transmission. At the highest $(11.8 \mathrm{~cm} / \mathrm{min})$ and lowest $\left(7.9 \times 10^{-4}\right.$ $\mathrm{cm} / \mathrm{min}$ ) fluxes the profiles are monotonic with distance and no saturation overshoot is observed, while all of the intermediate fluxes exhibit saturation overshoot. 


\subsection{Derivation of the Modified Buckley-Leverett Equation}

To better understand the problem, we go back to the origins of (1.1). Let

$$
S_{i}=\text { saturation of oil/water } \quad i=\mathrm{o}, \mathrm{w}
$$

where

$$
S_{o}+S_{w}=1
$$

that is, the medium is assumed to be completely saturated. The balance of mass yields

$$
\frac{d}{d t} \int_{x_{1}}^{x_{2}} \phi S_{i}(x, t) d x=q_{i}\left(x_{1}\right)-q_{i}\left(x_{2}\right) \quad \text { for any } x_{1}, x_{2}, \quad \text { and } i=0, w
$$

where

$$
\begin{aligned}
\phi & =\text { porosity of the medium } \\
& =\text { relative volume occupied by the pores, so lies between } 0 \text { and } 1, \\
q_{i} & =\text { discharge of oil/water. }
\end{aligned}
$$

Since $x_{1}, x_{2}$ are arbitrary, if $S_{i}$ and $q_{i}$ are smooth, we have the differential form of the conservation law

$$
\phi \frac{\partial S_{i}}{\partial t}+\frac{\partial q_{i}}{\partial x}=0
$$

Notice that due to the complete saturation assumption (1.7), we have that

$$
q_{o}+q_{w}=q=\text { const in space. }
$$


Throughout of this thesis, we consider it constant in time as well. The discharge of each phase is modeled by Darcy's law [20]

$$
q_{i}=-k \frac{k_{r i}\left(S_{i}\right)}{\mu_{i}} \frac{\partial P_{i}}{\partial x}, \quad i=o, w .
$$

where

$$
\begin{aligned}
k & =\text { absolute permeability }, \\
k_{r i} & =\text { relative permeability } \\
\mu_{i} & =\text { viscosity } \\
P_{i} & =\text { phase pressure }
\end{aligned}
$$

For the ease of notation, we denote

$$
\lambda_{i}=-k \frac{k_{r_{i}}\left(S_{i}\right)}{\mu_{i}}
$$

then 1.10 becomes

$$
q_{i}=\lambda_{i} \frac{\partial P_{i}}{\partial x}
$$

Instead of considering constant capillary pressure as adopted by the classical BL equation (1.1), Hassanizadeh and Gray [9] [10] have defined the dynamic capillary pressure as

$$
P_{c}=P_{o}-P_{w}=p_{c}\left(S_{w}\right)-\phi \tau \frac{\partial S_{w}}{\partial t}
$$

where $p_{c}\left(S_{w}\right)$ is the static capillary pressure and $\tau$ is a positive constant, and $\frac{\partial S_{w}}{\partial t}$ is the dynamic effects. To simplify the notation, let's write (1.13) as

$$
P_{o}-P_{w}=\text { r.h.s. }
$$


where

$$
\text { r.h.s. }=p_{c}\left(S_{w}\right)-\phi \tau \frac{\partial S_{w}}{\partial t},
$$

then

$$
\frac{\partial P_{o}}{\partial x}-\frac{\partial P_{w}}{\partial x}=\frac{\partial}{\partial x}[\text { r.h.s. }]
$$

Combine (1.12), we get

$$
\frac{q_{o}}{\lambda_{o}}-\frac{q_{w}}{\lambda_{w}}=\frac{\partial}{\partial x}[\text { r.h.s. }] .
$$

Notice that (1.9) gives

$$
\begin{aligned}
\frac{q_{o}}{\lambda_{o}}-\frac{q_{w}}{\lambda_{w}} & =\frac{q-q_{w}}{\lambda_{o}}-\frac{q_{w}}{\lambda_{w}} \\
& =\frac{q}{\lambda_{o}}-\frac{\lambda_{o}+\lambda_{w}}{\lambda_{o} \lambda_{w}} q_{w},
\end{aligned}
$$

and hence 1.15 becomes

$$
\frac{q}{\lambda_{o}}-\frac{\lambda_{o}+\lambda_{w}}{\lambda_{o} \lambda_{w}} q_{w}=\frac{\partial}{\partial x}[\text { r.h.s. }]
$$

and

$$
q_{w}=\frac{\lambda_{w}}{\lambda_{o}+\lambda_{w}} q-\frac{\lambda_{o} \lambda_{w}}{\lambda_{o}+\lambda_{w}} \frac{\partial}{\partial x}[\text { r.h.s. }] .
$$

Plugging (1.16) into the governing equation (1.8) for $S_{w}$, we get that

$$
\phi \frac{\partial S_{w}}{\partial t}+\frac{\partial}{\partial x}\left[\frac{\lambda_{w}}{\lambda_{o}+\lambda_{w}} q-\frac{\lambda_{o} \lambda_{w}}{\lambda_{o}+\lambda_{w}} \frac{\partial}{\partial x}[\text { r.h.s. }]\right]=0,
$$

and hence

$$
\frac{\partial S_{w}}{\partial t}+\frac{\partial}{\partial x}\left[\frac{\lambda_{w}}{\lambda_{o}+\lambda_{w}} \frac{q}{\phi}\right]=\frac{\partial}{\partial x}\left[\frac{\lambda_{o} \lambda_{w}}{\phi\left(\lambda_{o}+\lambda_{w}\right)} \frac{\partial}{\partial x}[\text { r.h.s. }]\right] .
$$


By the definition of $\lambda_{w}, \lambda_{o}$, and r.h.s. in (1.11) and (1.14) respectively, we have that

$$
\begin{aligned}
& \frac{\partial S_{w}}{\partial t}+\frac{\partial}{\partial x}\left[\frac{-k k_{r w}\left(S_{w}\right) / \mu_{w}}{-k k_{r_{o}}\left(S_{o}\right) / \mu_{o}-k k_{r w}\left(S_{w}\right) / \mu_{w}} \frac{q}{\phi}\right] \\
= & \frac{\partial}{\partial x}\left[\frac{\left(-k k_{r_{o}}\left(S_{o}\right) / \mu_{o}\right)\left(-k k_{r w}\left(S_{w}\right) / \mu_{w}\right)}{-k k_{r_{o}}\left(S_{o}\right) / \mu_{o}-k k_{r w}\left(S_{w}\right) / \mu_{w}} \frac{\partial}{\partial x}\left(\frac{p_{c}\left(S_{w}\right)}{\phi}-\tau \frac{\partial S_{w}}{\partial t}\right)\right] .
\end{aligned}
$$

Using Corey [6, 19] expressions,

$$
k_{r_{w}}\left(S_{w}\right)=S_{w}^{2}, \quad k_{r_{o}}\left(S_{o}\right)=S_{o}^{2}
$$

and combining with (1.7), we get

$$
\begin{aligned}
& \frac{\partial S_{w}}{\partial t}+\frac{\partial}{\partial x}\left[\frac{S_{w}^{2}}{S_{w}^{2}+\frac{\mu_{w}}{\mu_{o}}\left(1-S_{w}\right)^{2}} \frac{q}{\phi}\right] \\
= & -\frac{\partial}{\partial x}\left[\frac{k\left(1-S_{w}\right)^{2} S_{w}^{2}}{\mu_{w}\left(1-S_{w}\right)^{2}+\mu_{o} S_{w}^{2}} \frac{\partial}{\partial x}\left(\frac{p_{c}\left(S_{w}\right)}{\phi}-\tau \frac{\partial S_{w}}{\partial t}\right)\right] .
\end{aligned}
$$

If we rescale

$$
x \frac{\phi}{q} \rightarrow x
$$

and let

$$
u=S_{w}=\text { saturation of water, }
$$

we get

$$
\frac{\partial u}{\partial t}+\frac{\partial}{\partial x}\left[\frac{u^{2}}{u^{2}+M(1-u)^{2}}\right]=-\frac{\partial}{\partial x}\left[\frac{\phi^{2}}{q^{2}} \frac{k(1-u)^{2} u^{2}}{\mu_{w}(1-u)^{2}+\mu_{o} u^{2}} \frac{\partial}{\partial x}\left(\frac{p_{c}(u)}{\phi}-\tau \frac{\partial u}{\partial t}\right)\right],
$$

where

$$
M=\frac{\mu_{w}}{\mu_{o}}
$$


Equation (1.17) can be written as a more general form

$$
\frac{\partial u}{\partial t}+\frac{\partial f(u)}{\partial x}=-\frac{\partial}{\partial x}\left\{H(u) \frac{\partial}{\partial x}\left(J(u)-\tau \frac{\partial u}{\partial t}\right)\right\} .
$$

where

$$
f(u)=\frac{u^{2}}{u^{2}+M(1-u)^{2}}
$$

is the BL flux. In this thesis, we consider the linearized right hand side. In $(1.18)$ if we take

$$
\begin{aligned}
& H(u)=\epsilon^{2}, \\
& J(u)=-\frac{u}{\epsilon},
\end{aligned}
$$

or equivalently if we assume porosity $\phi$ is small in 1.17 by taking

$$
\begin{aligned}
\frac{\phi^{2}}{q^{2}} \frac{k(1-u)^{2} u^{2}}{\mu_{w}(1-u)^{2}+\mu_{o} u^{2}} & =\epsilon^{2}, \\
\frac{p_{c}(u)}{\phi} & =-\frac{u}{\epsilon},
\end{aligned}
$$

then the modified Buckley-Leverett equation (MBL) is obtained

$$
\frac{\partial u}{\partial t}+\frac{\partial f(u)}{\partial x}=\epsilon \frac{\partial^{2} u}{\partial x^{2}}+\epsilon^{2} \tau \frac{\partial^{2} u}{\partial x^{2} \partial t} .
$$

Note that, if $P_{c}$ in (1.13) is taken to be constant, then (1.17) gives the classical BL equation; while if the dispersive parameter $\tau$ is taken to be zero, or equivalently dynamic effect in the pressure difference between the two phases is neglected, then 1.19) gives the viscous BL equation, which still displays monotone water saturation profile. Thus, in addition to the classical second order viscous term $\epsilon u_{x x}$, the MBL 
equation 1.19 is an extension involving a third order mixed derivative term $\epsilon^{2} \tau u_{x x t}$. Van Dujin et al. 21] showed that the value $\tau$ is critical in determining the type of the profile. In particular, for certain Riemann problems, the solution profile of 1.19 is not monotone when $\tau$ is larger than the threshold value $\tau_{*}$, where $\tau_{*}$ was numerically determined to be 0.61 [21]. The non-monotonicity of the solution profile is consistent with the experimental observations 8$]$ as given in Figure 1.5 .

The classical BL equation (1.1) is hyperbolic, and the numerical schemes for hyperbolic equations have been well developed (e.g. [14, 15, 4, 5, 17, 12] ). The MBL equation (1.19), however, is pseudo-parabolic, we will illustrate how to extend the central schemes [17, 12] to solve (1.19) numerically. Unlike the finite domain of dependence for the classical BL equation (1.1), the domain of dependence for the MBL equation (1.19) is infinite. This naturally raises the question for the choice of computational domain. To answer this question, we will first study the MBL equation equipped with two types of domains and corresponding boundary conditions. One is the half line problem

$$
\begin{array}{rll}
u_{t}+(f(u))_{x}=\epsilon u_{x x}+\epsilon^{2} \tau u_{x x t} & \text { in } & Q=\{(x, t): x>0, t>0\} \\
u(x, 0)=u_{0}(x) & x \in[0, \infty) \\
u(0, t)=g_{u}(t), \quad \lim _{x \rightarrow \infty} u(x, t)=0 & t \in[0, \infty) \\
u_{0}(0)=g_{u}(0) & \text { compatibility condition }
\end{array}
$$


and the other one is the finite interval boundary value problem

$$
\begin{array}{rll}
v_{t}+(f(v))_{x}=\epsilon v_{x x}+\epsilon^{2} \tau v_{x x t} & \text { in } & \widetilde{Q}=\{(x, t): x \in(0, L), t>0\} \\
v(x, 0)=v_{0}(x) & & x \in[0, L] \\
v(0, t)=g_{v}(t), \quad v(L, t)=h(t) & t \in[0, \infty) \\
v_{0}(0)=g_{v}(0), \quad v_{0}(L)=h(0) & \text { compatibility condition. }
\end{array}
$$

Considering

$$
u_{0}(x)=\left\{\begin{array}{ll}
v_{0}(x) & \text { for } \quad x \in[0, L] \\
0 & \text { for } x \in[L,+\infty)
\end{array} \quad, \quad g_{u}(t)=g_{v}(t) \equiv g(t), \quad h(t) \equiv 0\right.
$$

we will show the relation between the solutions of problems 1.20 and (1.21). To the best knowledge of the author, there is no such study for MBL equation (1.19). Similar questions were answered for BBM equation [1, 2].

The organization of this thesis is as follows. Chapter 2 will bring forward the exact theory comparing the solutions of $(1.20)$ and $(1.21)$. The difference between the solutions of these two types of problems decays exponentially with respect to the length of the interval $L$ for practically interesting initial profiles. This provides a theoretical justification for the choice of the computational domain. In chapter 3 , high order central schemes will be developed for MBL equation in finite interval domain. We provide a detailed derivation on how to extend the central schemes [17, 12] for conservation laws to solve the MBL equation (1.19). The idea of adopting numerical schemes originally designed for hyperbolic equations to pseudo-parabolic equations is not restricted to central type schemes only $([22,23])$. The numerical results in chapter 4 show that the water saturation profile strongly depends on the dispersive 
parameter $\tau$ value as studied in [21]. For $\tau>\tau_{*}$, the MBL equation (1.19) gives nonmonotone water saturation profiles for certain Riemann problems as suggested by experimental observations [8]. In chapter 5, we show the two-dimensional extension of MBL equation and discuss the preliminary numerical solutions. Chapter 6 gives the conclusion of the paper and the possible future directions. 


\section{CHAPTER 2}

\section{THE HALF LINE PROBLEM VERSUS THE FINITE INTERVAL BOUNDARY VALUE PROBLEM}

Let $u(x, t)$ be the solution to the half line problem 1.20 , and let $v(x, t)$ be the solution to the finite interval boundary value problem (1.21). We consider the natural assumptions 1.22 . The existence of solution for both the half line problem (1.20) the finite interval boundary value problem (1.21) can be proven similar to that in [1]. We will therefore omit that proof. The goal of this chapter is to develop an estimate of the difference between $u$ and $v$ on the spatial interval $[0, L]$ at a given finite time $t$. The main result of this section is

Theorem 2.0.1 (The main Theorem). If $u_{0}(x)$ satisfies

$$
u_{0}(x)= \begin{cases}C_{u} & x \in\left[0, L_{0}\right] \\ 0 & x>L_{0}\end{cases}
$$

where $L_{0}<L$ and $C_{u}$, are positive constants, then

$$
\|u(\cdot, t)-v(\cdot, t)\|_{H_{L, \epsilon, \tau}^{1}} \leq D_{1 ; \epsilon, \tau}(t) e^{-\frac{\lambda L}{\epsilon \sqrt{\tau}}}+D_{2 ; \epsilon, \tau}(t) e^{-\frac{\lambda\left(L-L_{0}\right)}{\epsilon \sqrt{\tau}}}
$$

for some $0<\lambda<1, D_{1 ; \epsilon, \tau}(t)>0$ and $D_{2 ; \epsilon, \tau}(t)>0$, where

$$
\|Y(\cdot, t)\|_{H_{L, \epsilon, \tau}^{1}}:=\sqrt{\int_{0}^{L} Y(x, t)^{2}+\left(\epsilon \sqrt{\tau} Y_{x}(x, t)\right)^{2} d x} .
$$


Notice that the initial condition (2.1) we considered is the Riemann problem. Theorem 2.0.1 shows that the solution to the half line problem (1.20) can be approximated as accurately as one wants by the solution to the finite interval boundary value problem 1.21 in the sense that $D_{1 ; \epsilon, \tau}(t), D_{2 ; \epsilon, \tau}(t), \frac{\lambda L}{\epsilon \sqrt{\tau}}$ and $\frac{\lambda\left(L-L_{0}\right)}{\epsilon \sqrt{\tau}}$ can be controlled. To prove theorem 2.0.1, we first derive the implicit solution formulae for the half line problem and the finite interval boundary value problem in section 2.1 and section 2.2 respectively. The implicit solution formulae are in integral form, which are derived by separating the $x$-derivative from the $t$-derivative, and formally solving a first order linear ODE in $t$ and a second order non-homogeneous ODE in $x$. In section 2.3, we use Gronwall's inequality multiple times to obtain the desired result in theorem 2.0.1.

\subsection{Half Line Problem}

In this section, we derive the implicit solution formula for the half line problem $(1.20)$. For ease of reference, the equation is repeated here.

$$
\begin{array}{rll}
u_{t}+(f(u))_{x}=\epsilon u_{x x}+\epsilon^{2} \tau u_{x x t} & \text { in } & Q=\{(x, t): x>0, t>0\} \\
u(x, 0)=u_{0}(x) & x \in[0, \infty) \\
u(0, t)=g_{u}(t)=g(t), \quad \lim _{x \rightarrow \infty} u(x, t)=0 & t \in[0, \infty) \\
u_{0}(0)=g_{u}(0)=g(0) & \text { compatibility condition. }
\end{array}
$$


To solve 2.2, we first rewrite 2.2 by separating the $x$-derivative from the $t$ derivative,

$$
\left(I-\epsilon^{2} \tau \frac{\partial^{2}}{\partial x^{2}}\right)\left(u_{t}+\frac{1}{\epsilon \tau} u\right)=\frac{1}{\epsilon \tau} u-(f(u))_{x} .
$$

Notice that 2.3) can be viewed as a first order linear ODE in $t$. By multiplying integrating factor, we get

$$
\begin{aligned}
\left(I-\epsilon^{2} \tau \frac{\partial^{2}}{\partial x^{2}}\right)\left(e^{\frac{t}{\epsilon \tau}} u_{t}+e^{\frac{t}{\epsilon \tau}} \frac{1}{\epsilon \tau} u\right) & =\left(\frac{1}{\epsilon \tau} u-(f(u))_{x}\right) e^{\frac{t}{\epsilon \tau}} \\
\left(I-\epsilon^{2} \tau \frac{\partial^{2}}{\partial x^{2}}\right)\left(u e^{\frac{t}{\epsilon \tau}}\right)_{t} & =\left(\frac{1}{\epsilon \tau} u-(f(u))_{x}\right) e^{\frac{t}{\epsilon \tau}}
\end{aligned}
$$

We formally integrate 2.4 over $[0, t]$ to obtain

$$
\begin{aligned}
\left(I-\epsilon^{2} \tau \frac{\partial^{2}}{\partial x^{2}}\right)\left(u e^{\frac{t}{\epsilon \tau}}-u_{0}\right) & =\int_{0}^{t}\left(\frac{1}{\epsilon \tau} u-(f(u))_{x}\right) e^{\frac{s}{\epsilon \tau}} d s \\
\left(I-\epsilon^{2} \tau \frac{\partial^{2}}{\partial x^{2}}\right)\left(u-e^{-\frac{t}{\epsilon \tau}} u_{0}\right) & =\int_{0}^{t}\left(\frac{1}{\epsilon \tau} u-(f(u))_{x}\right) e^{-\frac{t-s}{\epsilon \tau}} d s .
\end{aligned}
$$

Furthermore, we let

$$
A=u-e^{-\frac{t}{\epsilon \tau}} u_{0}
$$

then (2.5) can be written as

$$
A-\epsilon^{2} \tau A^{\prime \prime}=\int_{0}^{t}\left(\frac{1}{\epsilon \tau} u-(f(u))_{x}\right) e^{-\frac{t-s}{\epsilon \tau}} d s \quad \text { where }{ }^{\prime}=\frac{\partial}{\partial x},
$$

i.e.

$$
A^{\prime \prime}-\frac{1}{\epsilon^{2} \tau} A=\int_{0}^{t}\left(-\frac{1}{\epsilon^{3} \tau^{2}} u+\frac{1}{\epsilon^{2} \tau}(f(u))_{x}\right) e^{-\frac{t-s}{\epsilon \tau}} d s
$$




\section{CHAPTER 2. HALF LINE V.S. FINITE INTERVAL DOMAIN}

Notice that (2.7) is a second-order non-homogeneous ODE in $x$-variable along with the boundary conditions

$$
\begin{aligned}
A(0, t) & =u(0, t)-e^{-\frac{t}{\epsilon \tau}} u_{0}(0) \\
& =g(t)-e^{-\frac{t}{\epsilon \tau}} g(0), \\
A(\infty, t) & =u(\infty, t)-e^{-\frac{t}{\epsilon \tau}} u_{0}(\infty)=0 .
\end{aligned}
$$

To solve (2.7), we first solve the corresponding linear homogeneous equation with the non-zero boundary conditions (2.8).

$$
\begin{aligned}
\left(A_{h}\right)^{\prime \prime}-\frac{1}{\epsilon^{2} \tau} A_{h} & =0, \\
A_{h}(0, t) & =g(t)-e^{-\frac{t}{\epsilon \tau}} g(0), \\
A_{h}(\infty, t) & =0,
\end{aligned}
$$

and the solution is

$$
A_{h}(x, t)=\left(g(t)-e^{-\frac{t}{\epsilon \tau}} g(0)\right) e^{-\frac{x}{\epsilon \sqrt{\tau}}} .
$$

We then find a particular solution for the non-homogeneous equation with zero boundary conditions

$$
\begin{aligned}
B^{\prime \prime}-\frac{1}{\epsilon^{2} \tau} B & =\int_{0}^{t}\left(-\frac{1}{\epsilon^{3} \tau^{2}} u+\frac{1}{\epsilon^{2} \tau}(f(u))_{x}\right) e^{-\frac{t-s}{\epsilon \tau}} d x, \\
B(0, t) & =0, \\
B(\infty, t) & =0 .
\end{aligned}
$$


We break the right hand side of (2.9) into two parts and consider them separately.

Let $B_{1}$ satisfy

$$
\begin{aligned}
B_{1}^{\prime \prime}-\frac{1}{\epsilon^{2} \tau} B_{1} & =\int_{0}^{t}-\frac{1}{\epsilon^{3} \tau^{2}} u e^{-\frac{t-s}{\epsilon \tau}} d x, \\
B_{1}(0, t) & =0 \\
B_{1}(\infty, t) & =0 .
\end{aligned}
$$

We look for the Green's function $G(x, \xi)(\xi \in(0, \infty))$ for $B_{1}$ that satisfies

$$
\begin{aligned}
G^{\prime \prime}-\frac{1}{\epsilon^{2} \tau} G & =\delta(x-\xi), \\
G(0, \xi) & =0, \\
G(\infty, \xi) & =0, \\
G\left(\xi^{-}, \xi\right) & =G\left(\xi^{+}, \xi\right), \quad \text { where }{ }^{\prime}=\frac{\partial}{\partial x} .
\end{aligned}
$$

Such a Green's function $G(x, \xi)$ is

$$
G(x, \xi)=\frac{\epsilon \sqrt{\tau}}{2}\left(e^{-\frac{x+\xi}{\epsilon \sqrt{\tau}}}-e^{-\frac{|x-\xi|}{\epsilon \sqrt{\tau}}}\right) .
$$

Hence,

$$
\begin{aligned}
B_{1}(x, t) & =-\frac{1}{\epsilon^{3} \tau^{2}} \int_{0}^{t} \int_{0}^{+\infty} G(x, \xi) u(\xi, s) e^{-\frac{t-s}{\epsilon \tau}} d \xi d s \\
& =-\frac{1}{2 \epsilon^{2} \tau \sqrt{\tau}} \int_{0}^{t} \int_{0}^{+\infty}\left(e^{-\frac{x+\xi}{\epsilon \sqrt{\tau}}}-e^{-\frac{|x-\xi|}{\epsilon \sqrt{\tau}}}\right) u(\xi, s) e^{-\frac{t-s}{\epsilon \tau}} d \xi d s
\end{aligned}
$$


Similarly, let $B_{2}$ satisfy

$$
\begin{aligned}
B_{2}^{\prime \prime}-\frac{1}{\epsilon^{2} \tau} B_{2} & =\int_{0}^{t} \frac{1}{\epsilon^{2} \tau}(f(u))_{x} e^{-\frac{t-s}{\epsilon \tau}} d s, \\
B_{2}(0, t) & =0 \\
B_{2}(\infty, t) & =0 .
\end{aligned}
$$

Since

$$
\begin{aligned}
& \int_{0}^{+\infty}(f(u))_{\xi} G(x, \xi) d \xi \\
= & \int_{0}^{+\infty} \frac{\epsilon \sqrt{\tau}}{2}(f(u))_{\xi}\left(e^{-\frac{x+\xi}{\epsilon \sqrt{\tau}}}-e^{-\frac{|x-\xi|}{\epsilon \sqrt{\tau}}}\right) d \xi \\
= & {\left[\frac{\epsilon \sqrt{\tau}}{2} f(u)\left(e^{-\frac{x+\xi}{\epsilon \sqrt{\tau}}}-e^{-\frac{|x-\xi|}{\epsilon \sqrt{\tau}}}\right)\right]_{\xi=0}^{\xi=+\infty} } \\
& -\int_{0}^{+\infty} \frac{\epsilon \sqrt{\tau}}{2} f(u)\left(-\frac{1}{\epsilon \sqrt{\tau}} e^{-\frac{x+\xi}{\epsilon \sqrt{\tau}}}-\frac{1}{\epsilon \sqrt{\tau}} \operatorname{sgn}(x-\xi) e^{-\frac{|x-\xi|}{\epsilon \sqrt{\tau}}}\right) d \xi \\
= & \frac{1}{2} \int_{0}^{+\infty} f(u)\left(e^{-\frac{x+\xi}{\epsilon \sqrt{\tau}}}+\operatorname{sgn}(x-\xi) e^{-\frac{|x-\xi|}{\epsilon \sqrt{\tau}}}\right) d \xi,
\end{aligned}
$$

let Kernel $K(x, \xi)$ where $\xi \in(0, \infty)$ be

$$
K(x, \xi)=\frac{1}{2}\left(e^{-\frac{x+\xi}{\epsilon \sqrt{\tau}}}+\operatorname{sgn}(x-\xi) e^{-\frac{|x-\xi|}{\epsilon \sqrt{\tau}}}\right)
$$

and hence $B_{2}(x, t)$ is

$$
\begin{aligned}
B_{2}(x, t) & =\frac{1}{\epsilon^{2} \tau} \int_{0}^{t} \int_{0}^{+\infty} K(x, \xi) f(u) e^{-\frac{t-s}{\epsilon \tau}} d \xi d s \\
& =\frac{1}{2 \epsilon^{2} \tau} \int_{0}^{t} \int_{0}^{+\infty}\left(e^{-\frac{x+\xi}{\sqrt{T}}}+\operatorname{sgn}(x-\xi) e^{-\frac{|x-\xi|}{\epsilon \sqrt{\tau}}}\right) f(u) e^{-\frac{t-s}{\epsilon \tau}} d \xi d s .
\end{aligned}
$$


Therefore, the solution for $(2.9)$ is

$$
\begin{aligned}
B(x, t)= & B_{1}(x, t)+B_{2}(x, t) \\
= & -\frac{1}{2 \epsilon^{2} \tau \sqrt{\tau}} \int_{0}^{t} \int_{0}^{+\infty}\left(e^{-\frac{x+\xi}{\epsilon \sqrt{\tau}}}-e^{-\frac{|x-\xi|}{\epsilon \sqrt{\tau}}}\right) u(\xi, s) e^{-\frac{t-s}{\epsilon \tau}} d \xi d s \\
& +\frac{1}{2 \epsilon^{2} \tau} \int_{0}^{t} \int_{0}^{+\infty}\left(e^{-\frac{x+\xi}{\epsilon \sqrt{\tau}}}+\operatorname{sgn}(x-\xi) e^{-\frac{|x-\xi|}{\epsilon \sqrt{\tau}}}\right) f(u) e^{-\frac{t-s}{\epsilon \tau}} d \xi d s .
\end{aligned}
$$

Therefore,

$$
\begin{aligned}
A(x, t) & =B(x, t)+A_{h}(x, t) \\
& =B(x, t)+\left(g(t)-e^{-\frac{t}{\epsilon \tau}} g(0)\right) e^{-\frac{x}{\epsilon \sqrt{\tau}}}
\end{aligned}
$$

By (2.6), we get the implicit solution formulae for $u(x, t)$

$$
\begin{aligned}
u(x, t)= & A(x, t)+e^{-\frac{t}{\epsilon \tau}} u_{0}(x) \\
= & B(x, t)+\left(g(t)-e^{-\frac{t}{\epsilon \tau}} g(0)\right) e^{-\frac{x}{\epsilon \sqrt{\tau}}}+e^{-\frac{t}{\epsilon \tau}} u_{0}(x) \\
= & -\frac{1}{2 \epsilon^{2} \tau \sqrt{\tau}} \int_{0}^{t} \int_{0}^{+\infty}\left(e^{-\frac{x+\xi}{\epsilon \sqrt{\tau}}}-e^{-\frac{|x-\xi|}{\epsilon \sqrt{\tau}}}\right) u(\xi, s) e^{-\frac{t-s}{\epsilon \tau}} d \xi d s \\
& +\frac{1}{2 \epsilon^{2} \tau} \int_{0}^{t} \int_{0}^{+\infty}\left(e^{-\frac{x+\xi}{\epsilon \sqrt{\tau}}}+\operatorname{sgn}(x-\xi) e^{-\frac{|x-\xi|}{\epsilon \sqrt{\tau}}}\right) f(u) e^{-\frac{t-s}{\epsilon \tau}} d \xi d s \\
& +\left(g(t)-e^{-\frac{t}{\epsilon \tau}} g(0)\right) e^{-\frac{x}{\epsilon \sqrt{\tau}}}+e^{-\frac{t}{\epsilon \tau}} u_{0}(x) .
\end{aligned}
$$

\subsection{Finite Interval Boundary Value Problem}

In this section, we derive the implicit solution formula for the finite interval boundary value problem 1.21. For ease of reference, the finite interval boundary value problem 
is repeated here.

$$
\begin{array}{rll}
v_{t}+(f(v))_{x}=\epsilon v_{x x}+\epsilon^{2} \tau v_{x x t} & \text { in } & \\
& v(x, 0)=v_{0}(x) & \\
& x \in[0, L] \\
v(0, t)=g_{v}(t)=g(t) \quad v(L, t)=h(t) & t \in[0, \infty) \\
v_{0}(0)=g_{v}(0)=g(0) & v_{0}(L)=h(0) & \text { compatibility condition. }
\end{array}
$$

The idea of solving (2.11) is the same as that of solving (2.2). The only difference is that the additional boundary condition $h(t)$ at $x=L$ in (2.11) gives different boundary conditions for the non-homogeneous ODE in $x$-variable.

To solve for the solution of 2.11), we first rewrite (2.11) by separating the $x$-derivative from the $t$-derivative, i.e.,

$$
\left(I-\epsilon^{2} \tau \frac{\partial^{2}}{\partial x^{2}}\right)\left(v_{t}+\frac{1}{\epsilon \tau} v\right)=\frac{1}{\epsilon \tau} v-(f(v))_{x}
$$

and then multiply the integrating factor

$$
\begin{aligned}
\left(I-\epsilon^{2} \tau \frac{\partial^{2}}{\partial x^{2}}\right)\left(e^{\frac{t}{\epsilon \tau}} v_{t}+e^{\frac{t}{\epsilon \tau}} \frac{1}{\epsilon \tau} v\right) & =\left(\frac{1}{\epsilon \tau} v-(f(v))_{x}\right) e^{\frac{t}{\epsilon \tau}} \\
\left(I-\epsilon^{2} \tau \frac{\partial^{2}}{\partial x^{2}}\right)\left(v e^{\frac{t}{\epsilon \tau}}\right)_{t} & =\left(\frac{1}{\epsilon \tau} v-(f(v))_{x}\right) e^{\frac{t}{\epsilon \tau}}
\end{aligned}
$$

Integrate 2.13 over $[0, t]$, we get

$$
\begin{aligned}
\left(I-\epsilon^{2} \tau \frac{\partial^{2}}{\partial x^{2}}\right)\left(v e^{\frac{t}{\epsilon \tau}}-v_{0}\right) & =\int_{0}^{t}\left(\frac{1}{\epsilon \tau} v-(f(v))_{x}\right) e^{\frac{s}{\epsilon \tau}} d s, \\
\left(I-\epsilon^{2} \tau \frac{\partial^{2}}{\partial x^{2}}\right)\left(v-e^{-\frac{t}{\epsilon \tau}} v_{0}\right) & =\int_{0}^{t}\left(\frac{1}{\epsilon \tau} v-(f(v))_{x}\right) e^{-\frac{t-s}{\epsilon \tau}} d s .
\end{aligned}
$$

Denote

$$
A^{L}=v-e^{-\frac{t}{\epsilon \tau}} v_{0}
$$


then $A^{L}$ satisfies

$$
A^{L}-\epsilon^{2} \tau\left(A^{L}\right)^{\prime \prime}=\int_{0}^{t}\left(-(f(v))_{x}+\frac{1}{\epsilon \tau} v\right) e^{-\frac{t-s}{\epsilon \tau}} d s \quad \text { where }{ }^{\prime}=\frac{\partial}{\partial x}
$$

i.e.,

$$
\left(A^{L}\right)^{\prime \prime}-\frac{1}{\epsilon^{2} \tau} A^{L}=\int_{0}^{t}\left(-\frac{1}{\epsilon^{3} \tau^{2}} v+\frac{1}{\epsilon^{2} \tau}(f(v))_{x}\right) e^{-\frac{t-s}{\epsilon \tau}} d x .
$$

The non-homogeneous second order ODE 2.16 has the following boundary conditions

$$
\begin{aligned}
A^{L}(0, t) & =v(0, t)-e^{-\frac{t}{\epsilon \tau}} v_{0}(0) \\
& =g(t)-e^{-\frac{t}{\epsilon \tau}} g(0), \\
A^{L}(L, t) & =v(L, t)-e^{-\frac{t}{\epsilon \tau}} v_{0}(L) \\
& =h(t)-e^{-\frac{t}{\epsilon \tau}} h(0) .
\end{aligned}
$$

The solution to the corresponding homogeneous equation

$$
\left(A_{h}^{L}\right)^{\prime \prime}-\frac{1}{\epsilon^{2} \tau} A_{h}^{L}=0
$$

with the boundary conditions

$$
\begin{aligned}
& A_{h}^{L}(0, t)=g(t)-e^{-\frac{t}{\epsilon \tau}} g(0) \\
& A_{h}^{L}(L, t)=h(t)-e^{-\frac{t}{\epsilon \tau}} h(0)
\end{aligned}
$$

is

$$
A_{h}^{L}=c_{1}(t) \phi_{1}(x)+c_{2}(t) \phi_{2}(x)
$$


where

$$
\begin{aligned}
c_{1}(t) & =g(t)-e^{-\frac{t}{\epsilon \tau}} g(0), \\
c_{2}(t) & =h(t)-e^{-\frac{t}{\epsilon \tau}} h(0), \\
\phi_{1}(x) & =\frac{e^{\frac{L-x}{\epsilon \sqrt{\tau}}}-e^{\frac{-L+x}{\epsilon \sqrt{\tau}}}}{e^{\frac{L}{\epsilon \sqrt{\tau}}}-e^{-\frac{L}{\epsilon \sqrt{\tau}}}}, \\
\phi_{2}(x) & =\frac{e^{\frac{x}{\epsilon \sqrt{\tau}}}-e^{-\frac{x}{\epsilon \sqrt{\tau}}}}{e^{\frac{L}{\epsilon \sqrt{\tau}}}-e^{-\frac{L}{\epsilon \sqrt{\tau}}}} .
\end{aligned}
$$

Now, we solve the corresponding non-homogeneous equation with zero boundary conditions:

$$
\begin{aligned}
\left(B^{L}\right)^{\prime \prime}-\frac{1}{\epsilon^{2} \tau} B^{L} & =\int_{0}^{t}\left(-\frac{1}{\epsilon^{3} \tau^{2}} v+\frac{1}{\epsilon^{2} \tau}(f(v))_{x}\right) e^{-\frac{t-s}{\epsilon \tau}} d x, \\
B^{L}(0, t) & =0 \\
B^{L}(L, t) & =0 .
\end{aligned}
$$

We break the right hand side of 2.21) into two parts and consider them separately. Let $B_{1}^{L}$ satisfy

$$
\begin{aligned}
\left(B_{1}^{L}\right)^{\prime \prime}-\frac{1}{\epsilon^{2} \tau} B_{1}^{L} & =\int_{0}^{t}-\frac{1}{\epsilon^{3} \tau^{2}} v e^{-\frac{t-s}{\epsilon \tau}} d x, \\
B_{1}^{L}(0, t) & =0, \\
B_{1}^{L}(L, t) & =0 .
\end{aligned}
$$


We look for the Green's function $G^{L}(x, \xi)$, where $\xi \in(0, L)$, for $B_{1}^{L}$ that satisfies

$$
\begin{aligned}
\left(G^{L}\right)^{\prime \prime}-\frac{1}{\epsilon^{2} \tau} G^{L} & =\delta(x-\xi), \\
G^{L}(0, \xi) & =0, \\
G^{L}(L, \xi) & =0, \\
G^{L}\left(\xi^{-}, \xi\right) & =G^{L}\left(\xi^{+}, \xi\right), \quad \text { where }{ }^{\prime}=\frac{\partial}{\partial x} .
\end{aligned}
$$

Such a Green's function $G^{L}(x, \xi)$ is

$$
G^{L}(x, \xi)=\frac{\epsilon \sqrt{\tau}}{2\left(e^{\frac{2 L}{\epsilon \sqrt{\tau}}}-1\right)}\left(e^{\frac{x+\xi}{\epsilon \sqrt{\tau}}}+e^{\frac{2 L-(x+\xi)}{\epsilon \sqrt{\tau}}}-e^{\frac{|x-\xi|}{\epsilon \sqrt{\tau}}}-e^{\frac{2 L-|x-\xi|}{\epsilon \sqrt{\tau}}}\right) .
$$

Hence,

$$
\begin{aligned}
B_{1}^{L}(x, t)= & -\frac{1}{\epsilon^{3} \tau^{2}} \int_{0}^{t} \int_{0}^{L} G_{1}^{L}(x, \xi) v(\xi, s) e^{-\frac{t-s}{\epsilon \tau}} d \xi d s \\
= & -\frac{1}{2 \epsilon^{2} \tau \sqrt{\tau}\left(e^{\frac{2 L}{\epsilon \sqrt{\tau}}}-1\right)} \int_{0}^{t} \int_{0}^{L} \\
& \quad\left(e^{\frac{x+\xi}{\epsilon \sqrt{\tau}}}+e^{\frac{2 L-(x+\xi)}{\epsilon \sqrt{\tau}}}-e^{\frac{|x-\xi|}{\epsilon \sqrt{\tau}}}-e^{\frac{2 L-|x-\xi|}{\epsilon \sqrt{\tau}}}\right) v(\xi, s) e^{-\frac{t-s}{\epsilon \tau}} d \xi d s .
\end{aligned}
$$

Similarly, let $B_{2}^{L}$ satisfy

$$
\begin{aligned}
\left(B_{2}^{L}\right)^{\prime \prime}-\frac{1}{\epsilon^{2} \tau} B_{2}^{L} & =\int_{0}^{t} \frac{1}{\epsilon^{2} \tau}(f(v))_{x} e^{-\frac{t-s}{\epsilon \tau}} d s, \\
B_{2}^{L}(0, t) & =0 \\
B_{2}^{L}(L, t) & =0 .
\end{aligned}
$$


Since

$$
\begin{aligned}
& \int_{0}^{L}(f(v))_{\xi} G_{1}^{L}(x, \xi) d \xi \\
&= \int_{0}^{L} \frac{\epsilon \sqrt{\tau}}{2\left(e^{\frac{2 L}{\epsilon \sqrt{\tau}}}-1\right)}(f(v))_{\xi}\left(e^{\frac{x+\xi}{\epsilon \sqrt{\tau}}}+e^{\frac{2 L-(x+\xi)}{\epsilon \sqrt{\tau}}}-e^{\frac{|x-\xi|}{\epsilon \sqrt{\tau}}}-e^{\frac{2 L-|x-\xi|}{\epsilon \sqrt{\tau}}}\right) d \xi \\
&= {\left[\frac{\epsilon \sqrt{\tau}}{2\left(e^{\frac{2 L}{\epsilon \sqrt{\tau}}}-1\right)} f(v)\left(e^{\frac{x+\xi}{\epsilon \sqrt{\tau}}}+e^{\frac{2 L-(x+\xi)}{\epsilon \sqrt{\tau}}}-e^{\frac{|x-\xi|}{\epsilon \sqrt{\tau}}}-e^{\frac{2 L-|x-\xi|}{\epsilon \sqrt{\tau}}}\right)\right]_{\xi=0}^{\xi=L} } \\
&-\int_{0}^{L} \frac{\epsilon \sqrt{\tau}}{2\left(e^{\frac{2 L}{\epsilon \sqrt{\tau}}}-1\right)} f(v)\left(\frac{1}{\epsilon \sqrt{\tau}} e^{\frac{x+\xi}{\sqrt{\tau}}}-\frac{1}{\epsilon \sqrt{\tau}} e^{\frac{2 L-(x+\xi)}{\epsilon \sqrt{\tau}}}\right. \\
&\left.\quad+\frac{1}{\epsilon \sqrt{\tau}} \operatorname{sgn}(x-\xi) e^{\frac{|x-\xi|}{\epsilon \sqrt{\tau}}}-\frac{1}{\epsilon \sqrt{\tau}} \operatorname{sgn}(x-\xi) e^{\frac{2 L-|x-\xi|}{\epsilon \sqrt{\tau}}}\right) d \xi \\
&=-\frac{1}{2\left(e^{\frac{2 L}{\epsilon \sqrt{\tau}}}-1\right)} \int_{0}^{L} f(v)\left(e^{\frac{x+\xi}{\epsilon \sqrt{\tau}}}-e^{\frac{2 L-(x+\xi)}{\epsilon \sqrt{\tau}}}\right. \\
&\left.\quad+\operatorname{sgn}(x-\xi) e^{\frac{|x-\xi|}{\epsilon \tau}}-\operatorname{sgn}(x-\xi) e^{\frac{2 L-|x-\xi|}{\epsilon \sqrt{\tau}}}\right) d \xi,
\end{aligned}
$$

let $K^{L}(x, \xi)$ where $\xi \in(0, L)$ be

$$
K^{L}(x, \xi)=-\frac{1}{2\left(e^{\frac{2 L}{\epsilon \sqrt{\tau}}}-1\right)}\left(e^{\frac{x+\xi}{\epsilon \sqrt{\tau}}}-e^{\frac{2 L-(x+\xi)}{\epsilon \sqrt{\tau}}}+\operatorname{sgn}(x-\xi) e^{\frac{|x-\xi|}{\epsilon \sqrt{\tau}}}-\operatorname{sgn}(x-\xi) e^{\frac{2 L-|x-\xi|}{\epsilon \sqrt{\tau}}}\right),
$$

and hence $B_{2}^{L}(x, t)$ is

$$
\begin{aligned}
B_{2}^{L}(x, t) & =\frac{1}{\epsilon^{2} \tau} \int_{0}^{t} \int_{0}^{L} K^{L}(x, \xi) f(v) e^{-\frac{t-s}{\epsilon \tau}} d \xi d s \\
& =-\frac{1}{2 \epsilon^{2} \tau\left(e^{\frac{2 L}{\epsilon \sqrt{\tau}}}-1\right)} \int_{0}^{t} \int_{0}^{+\infty} f(v) e^{-\frac{t-s}{\epsilon \tau}} \\
& \cdot\left(e^{\frac{x+\xi}{\epsilon \sqrt{\tau}}}-e^{\frac{2 L-(x+\xi)}{\epsilon \sqrt{\tau}}}+\operatorname{sgn}(x-\xi) e^{\frac{|x-\xi|}{\epsilon \sqrt{\tau}}}-\operatorname{sgn}(x-\xi) e^{\frac{2 L-|x-\xi|}{\epsilon \sqrt{\tau}}}\right) d \xi d s .
\end{aligned}
$$


Therefore, the solution for (2.21) is

$$
\begin{aligned}
B^{L}(x, t)= & B_{1}^{L}(x, t)+B_{2}^{L}(x, t) \\
= & -\frac{1}{2 \epsilon^{2} \tau \sqrt{\tau}\left(e^{\frac{2 L}{\epsilon \sqrt{\tau}}}-1\right)} \int_{0}^{t} \int_{0}^{L}\left(e^{\frac{x+\xi}{\epsilon \sqrt{\tau}}}+e^{\frac{2 L-(x+\xi)}{\epsilon \sqrt{\tau}}}-e^{\frac{|x-\xi|}{\epsilon \sqrt{\tau}}}-e^{\frac{2 L-|x-\xi|}{\epsilon \sqrt{\tau}}}\right) \\
& \cdot v(\xi, s) e^{-\frac{t-s}{\epsilon \tau}} d \xi d s \\
& -\frac{1}{2 \epsilon^{2} \tau\left(e^{\frac{2 L}{\epsilon \sqrt{\tau}}}-1\right)} \int_{0}^{t} \int_{0}^{L} f(v) e^{-\frac{t-s}{\epsilon \tau}} \\
& \cdot\left(e^{\frac{x+\xi}{\epsilon \sqrt{\tau}}}-e^{\frac{2 L-(x+\xi)}{\epsilon \sqrt{\tau}}}+\operatorname{sgn}(x-\xi) e^{\frac{|x-\xi|}{\epsilon \sqrt{\tau}}}-\operatorname{sgn}(x-\xi) e^{\frac{2 L-|x-\xi|}{\epsilon \sqrt{\tau}}}\right) d \xi d s .
\end{aligned}
$$

Therefore,

$$
\begin{aligned}
A^{L}(x, t) & =B^{L}(x, t)+A_{h}^{L}(x, t) \\
& =B^{L}(x, t)+\left(g(t)-e^{-\frac{t}{\epsilon \tau}} g(0)\right) \phi_{1}(x)+\left(h(t)-e^{-\frac{t}{\epsilon \tau}} h(0)\right) \phi_{2}(x) .
\end{aligned}
$$

By (2.14), we get the implicit solution formulae for $v(x, t)$

$$
\begin{aligned}
v(x, t)= & A^{L}(x, t)+e^{-\frac{t}{\epsilon \tau}} v_{0}(x) \\
= & B^{L}(x, t)+\left(g(t)-e^{-\frac{t}{\epsilon \tau}} g(0)\right) \phi_{1}(x)+\left(h(t)-e^{-\frac{t}{\epsilon \tau}} h(0)\right) \phi_{2}(x)+e^{-\frac{t}{\epsilon \tau}} v_{0}(x) \\
= & -\frac{1}{2 \epsilon^{2} \tau \sqrt{\tau}\left(e^{\frac{2 L}{\epsilon \sqrt{\tau}}}-1\right)} \int_{0}^{t} \int_{0}^{L}\left(e^{\frac{x+\xi}{\epsilon \sqrt{\tau}}}+e^{\frac{2 L-(x+\xi)}{\epsilon \sqrt{\tau}}}-e^{\frac{|x-\xi|}{\epsilon \sqrt{\tau}}}-e^{\frac{2 L-|x-\xi|}{\epsilon \sqrt{\tau}}}\right) \\
& -\frac{1}{2 \epsilon^{2} \tau\left(e^{\frac{2 L}{\epsilon \sqrt{\tau}}}-1\right)} \int_{0}^{t} \int_{0}^{L} f(\xi) e^{-\frac{t-s}{\epsilon \tau}} d \xi d s \\
& \cdot\left(e^{\frac{x+\xi}{\epsilon \sqrt{\tau}}}-e^{\frac{2 L-(x+\xi)}{\epsilon \sqrt{\tau}}}+\operatorname{sgn}(x-\xi) e^{\frac{|x-\xi|}{\epsilon \sqrt{\tau}}}-\operatorname{sgn}(x-\xi) e^{\frac{2 L-|x-\xi|}{\epsilon \sqrt{\tau}}}\right) d \xi d s \\
+ & \left(g(t)-e^{-\frac{t}{\epsilon \tau}} g(0)\right) \phi_{1}(x)+\left(h(t)-e^{-\frac{t}{\epsilon \tau}} h(0)\right) \phi_{2}(x)+e^{-\frac{t}{\epsilon \tau}} v_{0}(x) .
\end{aligned}
$$




\section{CHAPTER 2. HALF LINE V.S. FINITE INTERVAL DOMAIN}

\subsection{Comparisons}

With the implicit solution formulae for the half line problem and the finite interval boundary value problem derived in sections 2.1 and 2.2 respectively, we will prove in this section that the solution $u(x, t)$ to the half line problem can be approximated as accurately as one wants by the solution $v(x, t)$ to the finite interval boundary value problem as stated in Theorem 2.0.1.

The idea of the proof is to decompose $u(x, t)(v(x, t)$ respectively) into two parts: $U(x, t)$ and $u_{L}(x, t)\left(V(x, t)\right.$ and $v_{L}(x, t)$ respectively). $u_{L}(x, t)\left(v_{L}(x, t)\right.$ respectively) consists of terms involving the initial condition $u_{0}(x)\left(v_{0}(x)\right.$ respectively) and the boundary conditions $g(t)(g(t)$ and $h(t)$ respectively) for the governing equation 2.2) (2.11) respectively). $U(x, t)(V(x, t)$ respectively) enjoys zero initial condition and boundary conditions while satisfying a slightly different equation than 2.21 (2.11) respectively). We estimate the difference between $u(\cdot, t)$ and $v(\cdot, t)$ by estimating the differences between $u_{L}(\cdot, t)$ and $v_{L}(\cdot, t), U(\cdot, t)$ and $V(\cdot, t)$, then applying the triangle inequality.

In section 2.3.1, we will give the definitions of the decomposition of $u(x, t)(v(x, t)$ respectively) and a list of lemmas that will be used in the proof of Theorem 2.0.1. The

proof of the lemmas can be found in the appendix A. In addition, the norm $\|\cdot\|_{H_{L, \epsilon, \tau}^{1}}$ to be used in Theorem 2.0.1 will also be introduced in section 2.3.1. In section 2.3.2, we will prove a critical estimate which is essential to the proof of Theorem 2.0.1. In section 2.3.3, we will give the maximum difference $\left\|u_{L}(\cdot, t)-v_{L}(\cdot, t)\right\|_{\infty}$, and use it to derive $\left\|u_{L}(\cdot, t)-v_{L}(\cdot, t)\right\|_{H_{L, \epsilon, \tau}^{1}}$ and $\|U(\cdot, t)-V(\cdot, t)\|_{H_{L, \epsilon, \tau}^{1}}$ by using Gronwall's 
inequality. In the end, the ultimate difference $\|u(\cdot, t)-v(\cdot, t)\|_{H_{L, \epsilon, \tau}^{1}}$ will be derived based on the triangle inequality.

\subsubsection{Definitions and Lemmas}

To assist the proof of Theorem 2.0.1 in section 2.3.3, we introduce some new notations in this section. We first decompose $u(x, t)$ as sum of two terms $U(x, t)$ and $u_{L}(x, t)$, such that

$$
u(x, t)=U(x, t)+u_{L}(x, t) \quad x \in[0,+\infty)
$$

where

$$
u_{L}=e^{-\frac{t}{\epsilon \tau}} u_{0}(x)+c_{1}(t) e^{-\frac{x}{\epsilon \sqrt{\tau}}}+\left(u(L, t)-c_{1}(t) e^{-\frac{L}{\epsilon \sqrt{\tau}}}-e^{-\frac{t}{\epsilon \tau}} u_{0}(L)\right) \phi_{2}(x)
$$

and $c_{1}(t)$ and $\phi_{2}(x)$ are given in 2.17) and (2.20) respectively. With this definition, $u_{L}$ takes care of the initial condition $u_{0}(x)$ and boundary conditions $g(t)$ at $x=0$ and $x=L$ for $u(x, t)$. Then $U$ satisfies an equation slightly different from the equation $u$ satisfies in 2.2):

$$
\begin{aligned}
& U_{t}-\epsilon U_{x x}-\epsilon^{2} \tau U_{x x t} \\
= & \left(u_{t}-\epsilon u_{x x}-\epsilon^{2} \tau u_{x x t}\right)-\left(\left(u_{L}\right)_{t}-\epsilon\left(u_{L}\right)_{x x}-\epsilon^{2} \tau\left(u_{L}\right)_{x x t}\right) \\
= & -(f(u))_{x}+\frac{1}{\epsilon \tau} u_{L}(x, t) .
\end{aligned}
$$


In addition, $U(x, t)$ has zero initial condition and boundary conditions at $x=0$ and $x=L$, i.e.,

$$
\begin{gathered}
U(x, 0)=0, \\
U(0, t)=0, \\
U(L, t)=0 .
\end{gathered}
$$

Similarly, for $v(x, t)$, let

$$
v(x, t)=V(x, t)+v_{L}(x, t) \quad x \in[0, L]
$$

where

$$
v_{L}=e^{-\frac{t}{\epsilon \tau}} v_{0}(x)+c_{1}(t) \phi_{1}(x)+c_{2}(t) \phi_{2}(x)
$$

and $c_{1}(t), c_{2}(t)$ and $\phi_{1}(x), \phi_{2}(x)$ are given in 2.172 .18$)$ and 2.19 2.20 respectively. With this definition, $v_{L}$ takes care of the initial condition $v_{0}(x)$ and boundary conditions $g(t)$ and $h(t)$ at $x=0$ and $x=L$ for $v(x, t)$. Then $V$ satisfies an equation slightly different from the equation $v$ satisfies in 2.11):

$$
\begin{aligned}
& V_{t}-\epsilon V_{x x}-\epsilon^{2} \tau V_{x x t} \\
= & \left(v_{t}-\epsilon v_{x x}-\epsilon^{2} \tau v_{x x t}\right)-\left(\left(v_{L}\right)_{t}-\epsilon\left(v_{L}\right)_{x x}-\epsilon^{2} \tau\left(v_{L}\right)_{x x t}\right) \\
= & -(f(v))_{x}+\frac{1}{\epsilon \tau} v_{L}(x, t) .
\end{aligned}
$$

In addition, $V(x, t)$ has zero initial condition and boundary conditions at $x=0$ and $x=L$, i.e.,

$$
\begin{aligned}
& V(x, 0)=0, \\
& V(0, t)=0, \\
& V(L, t)=0 .
\end{aligned}
$$


Since, in the end, we want to study the difference between $U(x, t)$ and $V(x, t)$, we define

$$
W(x, t)=V(x, t)-U(x, t) \quad \text { for } \quad x \in[0, L] .
$$

Because of 2.23 and 2.26, we have

$$
W_{t}-\epsilon W_{x x}-\epsilon^{2} \tau W_{x x t}=-(f(v)-f(u))_{x}+\frac{1}{\epsilon \tau}\left(v_{L}-u_{L}\right) .
$$

In lieu of (2.24) and (2.27), $W(x, t)$ also has zero initial condition and boundary conditions at $x=0$ and $x=L$, i.e.,

$$
\begin{gathered}
W(x, 0)=0, \\
W(0, t)=0, \\
W(L, t)=0 .
\end{gathered}
$$

Now, in order to estimate $\|u-v\|$, we can estimate $\|W\|=\|V-U\|$ and estimate $\left\|u_{L}-v_{L}\right\|$ separately. These estimates are done in section 2.3.3.

Next, we state the lemmas needed in the proof of Theorem 2.0.1. The proof of the lemmas can be found in the appendix A. In all the lemmas, we assume $0<\lambda<1$ and $u_{0}(x)$ satisfies

$$
u_{0}(x)= \begin{cases}C_{u} & x \in\left[0, L_{0}\right] \\ 0 & x>L_{0}\end{cases}
$$

where $L_{0}<L$ and $C_{u}$ are positive constants. Notice that the constraint $\lambda \in(0,1)$ is crucial in Lemmmas 2.3 .2 and 2.3 .3 ,

Lemma 2.3.1. $f(u)=\frac{u^{2}}{u^{2}+M(1-u)^{2}} \leq D u$ where $D=\frac{f(\alpha)}{\alpha}$ and $\alpha=\sqrt{\frac{M}{M+1}}$. 
Lemma 2.3.2. $\quad$ (i) $\int_{0}^{+\infty}\left|e^{-\frac{x+\xi}{\sqrt{\tau}}}-e^{-\frac{|x-\xi|}{\epsilon \sqrt{\tau}}}\right| e^{\frac{\lambda x-\lambda \xi}{\epsilon \sqrt{\tau}}} d \xi \leq \frac{2 \epsilon \sqrt{\tau}}{1-\lambda^{2}}$.

(ii) $\int_{0}^{+\infty}\left|e^{-\frac{x+\xi}{\epsilon \sqrt{\tau}}}-e^{-\frac{|x-\xi|}{\epsilon \sqrt{\tau}}}\right| e^{\frac{\lambda x-\xi}{\epsilon \sqrt{\tau}}} d \xi \leq \frac{\epsilon \sqrt{\tau}}{e(1-\lambda)}$.

(iii) $\int_{0}^{+\infty}\left|e^{-\frac{x+\xi}{\epsilon \sqrt{\tau}}}-e^{-\frac{|x-\xi|}{\sqrt{\tau}}}\right| e^{\frac{\lambda x}{\epsilon \sqrt{\tau}}}\left|u_{0}(\xi)\right| d \xi \leq 2 C_{u} \epsilon \sqrt{\tau} e^{\frac{\lambda L_{0}}{\epsilon \sqrt{\tau}}}$.

Lemma 2.3.3. $\quad$ (i) $\int_{0}^{+\infty}\left|e^{-\frac{x+\xi}{\epsilon \sqrt{\tau}}}+\operatorname{sgn}(x-\xi) e^{-\frac{|x-\xi|}{\epsilon \sqrt{\tau}}}\right| e^{\frac{\lambda x-\lambda \xi}{\epsilon \sqrt{\tau}}} d \xi \leq \frac{2 \epsilon \sqrt{\tau}}{1-\lambda^{2}}$.

(ii) $\int_{0}^{+\infty}\left|e^{-\frac{x+\xi}{\epsilon \sqrt{\tau}}}+\operatorname{sgn}(x-\xi) e^{-\frac{|x-\xi|}{\epsilon \sqrt{\tau}}}\right| e^{\frac{\lambda x-\xi}{\epsilon \sqrt{\tau}}} d \xi \leq \epsilon \sqrt{\tau}+\frac{\epsilon \sqrt{\tau}}{e(1-\lambda)}$.

(iii) $\int_{0}^{+\infty}\left|e^{-\frac{x+\xi}{\epsilon \sqrt{\tau}}}+\operatorname{sgn}(x-\xi) e^{-\frac{|x-\xi|}{\epsilon \sqrt{\tau}}}\right| e^{\frac{\lambda x}{\epsilon \sqrt{\tau}}}\left|u_{0}(\xi)\right| d \xi \leq 2 C_{u} \epsilon \sqrt{\tau} e^{\frac{\lambda L_{0}}{\epsilon \sqrt{\tau}}}$.

Lemma 2.3.4. (i) $\left|\phi_{1}(x)-e^{-\frac{x}{\epsilon \sqrt{\tau}}}\right|=e^{-\frac{L}{\epsilon \sqrt{\tau}}}\left|\phi_{2}(x)\right|$.

(ii) $\left|\phi_{2}(x)\right| \leq 1$ for $x \in[0, L]$.

(iii) $\left|\phi_{2}^{\prime}(x)\right| \leq \frac{2}{\epsilon \sqrt{\tau}}$ if $\epsilon \ll 1$ for $x \in[0, L]$.

Last but not least, the norm that we will use in Theorem 2.0.1 and its proof is

$$
\|Y(\cdot, t)\|_{H_{L, \epsilon, \tau}^{1}}:=\sqrt{\int_{0}^{L} Y(x, t)^{2}+\left(\epsilon \sqrt{\tau} Y_{x}(x, t)\right)^{2} d x} .
$$

\subsubsection{A Proposition}

In this section, we will give a critical estimate, which is essential in the calculation of maximum difference $\left\|u_{L}(\cdot, t)-v_{L}(\cdot, t)\right\|_{\infty}$ in section 2.3.3. By comparing $u_{L}(x, t)$ and $v_{L}(x, t)$ given in 2.22 and 2.25) respectively, it is clear that the coefficient $u(L, t)-c_{1}(t) e^{-\frac{L}{\epsilon \sqrt{\tau}}}-e^{-\frac{t}{\epsilon \tau}} u_{0}(L)$ for $\phi_{2}(x)$ appeared in 2.22 needs to be compared 
with the corresponding coefficient $c_{2}(t)$ for $\phi_{2}(x)$ appeared in 2.25). In this section, we will find a bound for $u(L, t)-c_{1}(t) e^{-\frac{L}{\epsilon \sqrt{\tau}}}-e^{-\frac{t}{\epsilon \tau}} u_{0}(L)$ as follows

$$
\left|u(L, t)-c_{1}(t) e^{-\frac{L}{\epsilon \sqrt{\tau}}}-e^{-\frac{t}{\epsilon \tau}} u_{0}(L)\right| \leq a_{\tau}(t) e^{\frac{b_{\tau} t}{\epsilon \tau}} e^{-\frac{\lambda L}{\epsilon \sqrt{\tau}}}+c_{\tau} \frac{t}{\epsilon \tau} e^{\frac{\left(b_{\tau}-1\right) t}{\epsilon \tau}} e^{-\frac{\lambda\left(L-L_{0}\right)}{\epsilon \sqrt{\tau}}}
$$

for some parameter-dependent constants $a_{\tau}, b_{\tau}$ and $c_{\tau}$. The idea of the proof is to define a space-dependent function

$$
U_{c_{2}}(x, t)=u(x, t)-c_{1}(t) e^{-\frac{x}{\epsilon \sqrt{\tau}}}-e^{-\frac{t}{\epsilon \tau}} u_{0}(x)
$$

and show that $U_{c_{2}}(x, t)$ decays exponentially with respect to $x$ by using Gronwall's inequality and then evaluate $U_{c_{2}}$ at $x=L$ to obtain (2.32).

Based on the implicit solution formula (2.10) derived in section 2.1, we have

$$
\begin{aligned}
U_{c_{2}}(x, t)= & -\frac{1}{2 \epsilon^{2} \tau \sqrt{\tau}} \int_{0}^{t} \int_{0}^{+\infty}\left(e^{-\frac{x+\xi}{\epsilon \sqrt{\tau}}}-e^{-\frac{|x-\xi|}{\epsilon \sqrt{\tau}}}\right) u(\xi, s) e^{-\frac{t-s}{\epsilon \tau}} d \xi d s \\
& +\frac{1}{2 \epsilon^{2} \tau} \int_{0}^{t} \int_{0}^{+\infty}\left(e^{-\frac{x+\xi}{\epsilon \sqrt{\tau}}}+\operatorname{sgn}(x-\xi) e^{-\frac{|x-\xi|}{\epsilon \sqrt{\tau}}}\right) f(u) e^{-\frac{t-s}{\epsilon \tau}} d \xi d s,
\end{aligned}
$$

and based on the relationship between $U_{c_{2}}$ and $u$ given in (2.33), we have

$$
\begin{aligned}
U_{c_{2}}(x, t)=- & \frac{1}{2 \epsilon^{2} \tau \sqrt{\tau}}\left[\int_{0}^{t} \int_{0}^{+\infty}\left(e^{-\frac{x+\xi}{\epsilon \sqrt{\tau}}}-e^{-\frac{|x-\xi|}{\epsilon \sqrt{\tau}}}\right) U_{c_{2}}(\xi, s) e^{-\frac{t-s}{\epsilon \tau}} d \xi d s\right. \\
& +\int_{0}^{t} \int_{0}^{+\infty}\left(e^{-\frac{x+\xi}{\epsilon \sqrt{\tau}}}-e^{-\frac{|x-\xi|}{\epsilon \sqrt{\tau}}}\right) c_{1}(s) e^{-\frac{\xi}{\epsilon \sqrt{\tau}}} e^{-\frac{t-s}{\epsilon \tau}} d \xi d s \\
& \left.+\int_{0}^{t} \int_{0}^{+\infty}\left(e^{-\frac{x+\xi}{\epsilon \sqrt{\tau}}}-e^{-\frac{|x-\xi|}{\epsilon \sqrt{\tau}}}\right) u_{0}(\xi) e^{-\frac{s}{\epsilon \tau}} e^{-\frac{t-s}{\epsilon \tau}} d \xi d s\right] \\
& +\frac{1}{2 \epsilon^{2} \tau} \int_{0}^{t} \int_{0}^{+\infty}\left(e^{-\frac{x+\xi}{\epsilon \sqrt{\tau}}}+\operatorname{sgn}(x-\xi) e^{-\frac{|x-\xi|}{\epsilon \sqrt{\tau}}}\right) f(u) e^{-\frac{t-s}{\epsilon \tau}} d \xi d s
\end{aligned}
$$


and based on Lemma 2.3.1, we can get an inequality in terms of $U_{c_{2}}$

$$
\begin{aligned}
\left|U_{c_{2}}(x, t)\right| \leq & \frac{1}{2 \epsilon^{2} \tau \sqrt{\tau}}\left[\int_{0}^{t} \int_{0}^{+\infty}\left|e^{-\frac{x+\xi}{\epsilon \sqrt{\tau}}}-e^{-\frac{|x-\xi|}{\epsilon \sqrt{\tau}}}\right|\left|U_{c_{2}}(\xi, s)\right| e^{-\frac{t-s}{\epsilon \tau}} d \xi d s\right. \\
& +\int_{0}^{t} \int_{0}^{+\infty}\left|e^{-\frac{x+\xi}{\epsilon \sqrt{\tau}}}-e^{-\frac{\mid x-\xi \xi}{\epsilon \sqrt{\tau}}}\right|\left|c_{1}(s)\right| e^{-\frac{\xi}{\epsilon \sqrt{\tau}}} e^{-\frac{t-s}{\epsilon \tau}} d \xi d s \\
& \left.+\int_{0}^{t} \int_{0}^{+\infty}\left|e^{-\frac{x+\xi}{\epsilon \sqrt{\tau}}}-e^{-\frac{|x-\xi|}{\epsilon \sqrt{\tau}}}\right|\left|u_{0}(\xi)\right| e^{-\frac{t}{\epsilon \tau}} d \xi d s\right] \\
+ & \frac{D}{2 \epsilon^{2} \tau}\left[\int_{0}^{t} \int_{0}^{+\infty}\left|e^{-\frac{x+\xi}{\epsilon \sqrt{\tau}}}+\operatorname{sgn}(x-\xi) e^{-\frac{|x-\xi|}{\epsilon \sqrt{\tau}}}\right|\left|U_{c_{2}}(\xi, s)\right| e^{-\frac{t-s}{\epsilon \tau}} d \xi d s\right. \\
& +\int_{0}^{t} \int_{0}^{+\infty}\left|e^{-\frac{x+\xi}{\epsilon \sqrt{\tau}}}+\operatorname{sgn}(x-\xi) e^{-\frac{|x-\xi|}{\epsilon \sqrt{\tau}}}\right|\left|c_{1}(s)\right| e^{-\frac{\xi}{\epsilon \sqrt{\tau}}} e^{-\frac{t-s}{\epsilon \tau}} d \xi d s \\
& \left.+\int_{0}^{t} \int_{0}^{+\infty}\left|e^{-\frac{x+\xi}{\epsilon \sqrt{\tau}}}+\operatorname{sgn}(x-\xi) e^{-\frac{|x-\xi|}{\epsilon \sqrt{\tau}}}\right|\left|u_{0}(\xi)\right| e^{-\frac{t}{\epsilon \tau}} d \xi d s\right] .
\end{aligned}
$$

To show that $U_{c_{2}}(x, t)$ decays exponentially with respect to $x$, we first pull out an exponential term by writing

$$
U_{c_{2}}(x, t)=e^{-\frac{\lambda x}{\epsilon \sqrt{\tau}}} e^{-\frac{t}{\epsilon \tau}} \tilde{U}(x, t),
$$

where $0<\lambda<1$, such that

$$
\tilde{U}(x, t)=e^{\frac{\lambda x}{\epsilon \sqrt{\tau}}} e^{\frac{t}{\epsilon \tau}} U_{c_{2}}(x, t),
$$


then 2.34 can be rewritten in terms of $\tilde{U}(x, t)$ as follows

$$
\begin{aligned}
|\tilde{U}(x, t)| \leq & \frac{1}{2 \epsilon^{2} \tau \sqrt{\tau}}\left[\int_{0}^{t} \int_{0}^{+\infty}\left|e^{-\frac{x+\xi}{\epsilon \sqrt{\tau}}}-e^{-\frac{|x-\xi|}{\epsilon \sqrt{\tau}}}\right| e^{\frac{\lambda x-\lambda \xi}{\epsilon \sqrt{\tau}}}|\tilde{U}(\xi, s)| d \xi d s\right. \\
& +\int_{0}^{t} \int_{0}^{+\infty}\left|e^{-\frac{x+\xi}{\epsilon \sqrt{\tau}}}-e^{-\frac{|x-\xi|}{\epsilon \sqrt{\tau}}}\right|\left|c_{1}(s)\right| e^{\frac{\lambda x-\xi}{\epsilon \sqrt{\tau}}} e^{\frac{s}{\epsilon \tau}} d \xi d s \\
& \left.+\int_{0}^{t} \int_{0}^{+\infty}\left|e^{-\frac{x+\xi}{\epsilon \sqrt{\tau}}}-e^{-\frac{|x-\xi|}{\epsilon \sqrt{\tau}}}\right| e^{\frac{\lambda x}{\epsilon \sqrt{\tau}}}\left|u_{0}(\xi)\right| d \xi d s\right] \\
+ & \frac{D}{2 \epsilon^{2} \tau}\left[\int_{0}^{t} \int_{0}^{+\infty}\left|e^{-\frac{x+\xi}{\epsilon \sqrt{\tau}}}+\operatorname{sgn}(x-\xi) e^{-\frac{|x-\xi|}{\epsilon \sqrt{\tau}}}\right| e^{\frac{\lambda x-\lambda \xi}{\epsilon \sqrt{\tau}}} \mid \tilde{U}_{(\xi, s) \mid d \xi d s}^{t}\right. \\
& +\int_{0}^{t} \int_{0}^{+\infty}\left|e^{-\frac{x+\xi}{\epsilon \sqrt{\tau}}}+\operatorname{sgn}(x-\xi) e^{-\frac{|x-\xi|}{\epsilon \sqrt{\tau}}}\right|\left|c_{1}(s)\right| e^{\frac{\lambda x-\xi}{\epsilon \sqrt{\tau}}} e^{\frac{s}{\epsilon \tau}} d \xi d s \\
& \left.+\int_{0}^{t} \int_{0}^{+\infty}\left|e^{-\frac{x+\xi}{\epsilon \sqrt{\tau}}}+\operatorname{sgn}(x-\xi) e^{-\frac{|x-\xi|}{\epsilon \sqrt{\tau}}}\right| e^{\frac{\lambda x}{\epsilon \sqrt{\tau}}}\left|u_{0}(\xi)\right| d \xi d s\right] .
\end{aligned}
$$

Because of Lemmas 2.3.2 2.3.3 we can get the following estimate for $|\tilde{U}(\cdot, t)|_{\infty}$ based on (2.36) :

$$
\begin{aligned}
&|\tilde{U}(\cdot, t)|_{\infty} \leq \frac{1}{2 \epsilon^{2} \tau \sqrt{\tau}} {\left[\frac{2 \epsilon \sqrt{\tau}}{1-\lambda^{2}} \int_{0}^{t}|\tilde{U}(\cdot, s)|_{\infty} d s+\frac{\epsilon \sqrt{\tau}}{e(1-\lambda)} \int_{0}^{t}\left|c_{1}(s)\right| e^{\frac{s}{\epsilon \tau}} d s\right.} \\
&\left.+2 C_{u} \epsilon \sqrt{\tau} e^{\frac{\lambda L_{0}}{\epsilon \sqrt{\tau}}} \int_{0}^{t} 1 d s\right] \\
&+\frac{D}{2 \epsilon^{2} \tau}\left[\frac{2 \epsilon \sqrt{\tau}}{1-\lambda^{2}} \int_{0}^{t}|\tilde{U}(\cdot, s)|_{\infty} d s+\epsilon \sqrt{\tau}\left(1+\frac{1}{e(1-\lambda)}\right) \int_{0}^{t}\left|c_{1}(s)\right| e^{\frac{s}{\epsilon \tau}} d s\right. \\
&\left.\quad+2 C_{u} \epsilon \sqrt{\tau} e^{\frac{\lambda L_{0}}{\epsilon \sqrt{\tau}}} \int_{0}^{t} 1 d s\right] \\
& \leq \int_{0}^{t} \frac{b_{\tau}}{\epsilon \tau}|\tilde{U}(\cdot, s)|_{\infty} d s+\int_{0}^{t} \frac{\tilde{a}_{\tau}(s)}{\epsilon \tau} d s,
\end{aligned}
$$


where

$$
\begin{aligned}
b_{\tau} & =\frac{1+D \sqrt{\tau}}{1-\lambda^{2}} \\
\tilde{a}_{\tau}(t) & =a_{\tau} e^{\frac{t}{\epsilon \tau}}+c_{\tau} e^{\frac{\lambda L_{0}}{\epsilon \sqrt{\tau}}} \\
a_{\tau} & =\frac{\left|c_{1}(\cdot)\right|_{\infty}(1+D \sqrt{\tau}(e(1-\lambda)+1))}{2 e(1-\lambda)} \\
c_{\tau} & =C_{u}(1+D \sqrt{\tau})
\end{aligned}
$$

By Gronwall's inequality, inequality (2.37) gives that

$$
\begin{aligned}
|\tilde{U}(\cdot, t)|_{\infty} & \leq \int_{0}^{t} \frac{\tilde{a}_{\tau}(t-s)}{\epsilon \tau} e^{\frac{b_{\tau}(t-s)}{\epsilon \tau}} d s \\
& \leq\left(a_{\tau} e^{\frac{t}{\epsilon \tau}}+c_{\tau} \frac{t}{\epsilon \tau} e^{\frac{\lambda L_{0}}{\epsilon \sqrt{\tau}}}\right) e^{\frac{b_{\tau} t}{\epsilon \tau}} .
\end{aligned}
$$

Hence

$$
\begin{aligned}
\left|U_{c_{2}}(x, t)\right| & \leq|\tilde{U}(\cdot, t)|_{\infty} e^{\frac{-\lambda x}{\epsilon \sqrt{\tau}}} e^{-\frac{t}{\epsilon \tau}} \\
& \leq\left(a_{\tau} e^{\frac{t}{\epsilon \tau}}+c_{\tau} \frac{t}{\epsilon \tau} e^{\frac{\lambda L_{0}}{\epsilon \sqrt{\tau}}}\right) e^{\frac{b_{t} t}{\epsilon \tau}} e^{\frac{-\lambda x}{\epsilon \tau}} e^{-\frac{t}{\epsilon \tau}}
\end{aligned}
$$

i.e., $U_{c_{2}}(x, t)$ decays exponentially with respect to $x$. In particular, when $x=L$, we have

$$
\left|U_{c_{2}}(L, t)\right| \leq a_{\tau} e^{\frac{b_{\tau} t}{\epsilon \tau}} e^{-\frac{\lambda L}{\epsilon \sqrt{\tau}}}+c_{\tau} \frac{t}{\epsilon \tau} e^{\frac{\left(b_{\tau}-1\right) t}{\epsilon \tau}} e^{-\frac{\lambda\left(L-L_{0}\right)}{\epsilon \sqrt{\tau}}}
$$

as given in 2.32).

\subsubsection{Proof of Theorem 2.0.1}

In this section, we will first find the maximum difference of $\left\|u_{L}(\cdot, t)-v_{L}(\cdot, t)\right\|_{\infty}$ in proposition 2.3.5, then we will derive $\left\|u_{L}(\cdot, t)-v_{L}(\cdot, t)\right\|_{H_{L, \epsilon, \tau}^{1}}$, and $\|W(\cdot, t)\|_{H_{L, \epsilon, \tau}^{1}}=$ 
$\|U(\cdot, t)-V(\cdot, t)\|_{H_{L, \epsilon, \tau}^{1}}$ in propositions 2.3.6 and 2.3.7 respectively, and in turn we will get $\|u(\cdot, t)-v(\cdot, t)\|_{H_{L, \epsilon, \tau}^{1}}$ in theorem 2.3.8.

Proposition 2.3.5. If $u_{0}(x)$ satisfies 2.30), then $\left\|u_{L}-v_{L}\right\|_{\infty} \leq E_{1 ; \epsilon, \tau}(t) e^{-\frac{\lambda L}{\epsilon \sqrt{T}}}+$ $E_{2 ; \epsilon, \tau}(t) e^{-\frac{\lambda\left(L-L_{0}\right)}{\sqrt{\tau} \tau}}$ where $E_{1 ; \epsilon, \tau}(t)=\left|c_{1}(\cdot)\right|_{\infty}+a_{\tau} e^{\frac{b_{\tau} t}{\epsilon \tau}}$ and $E_{2 ; \epsilon, \tau}(t)=c_{\tau} \frac{t}{\epsilon \tau} e^{\frac{\left(b_{\tau}-1\right) t}{\epsilon \tau}}$.

Proof. By the definition of $u_{L}$ and $v_{L}$ given in (2.22) and (2.25) and the assumption that $u_{0}(x)=v_{0}(x)$ for $x \in[0, L]$, we can get their difference

$$
u_{L}(x, t)-v_{L}(x, t)=c_{1}(t)\left(e^{-\frac{x}{\epsilon \sqrt{\tau}}}-\phi_{1}(x)\right)+\left(U_{c_{2}}(L, t)-h(t)+e^{-\frac{t}{\epsilon \tau}} h(0)\right) \phi_{2}(x)
$$

Combining Lemmas 2.3.4(1), 2.3.4(ii), inequality (2.38), and $h(t) \equiv 0$, we have

$$
\begin{aligned}
\left\|u_{L}(\cdot, t)-v_{L}(\cdot, t)\right\|_{\infty} & \leq\left|c_{1}(t)\right| e^{-\frac{L}{\epsilon \sqrt{\tau}}}+\left|U_{c_{2}}(L, t)\right| \\
& \leq E_{1 ; \epsilon, \tau}(t) e^{-\frac{\lambda L}{\epsilon \sqrt{\tau}}}+E_{2 ; \epsilon, \tau}(t) e^{-\frac{\lambda\left(L-L_{0}\right)}{\epsilon \sqrt{\tau}}}
\end{aligned}
$$

where

$$
\begin{aligned}
E_{1 ; \epsilon, \tau}(t) & =\left|c_{1}(\cdot)\right|_{\infty}+a_{\tau} e^{\frac{b_{\tau} t}{\epsilon \tau}}, \\
E_{2 ; \epsilon, \tau}(t) & =c_{\tau} \frac{t}{\epsilon \tau} e^{\frac{\left(b_{\tau}-1\right) t}{\epsilon \tau}} .
\end{aligned}
$$

Proposition 2.3.6. If $u_{0}(x)$ satisfies 2.30), and $E_{1 ; \epsilon, \tau}(t), E_{2 ; \epsilon, \tau}(t)$ are as in proposition 2.3.5, then $\left\|u_{L}(\cdot, t)-v_{L}(\cdot, t)\right\|_{H_{L, \epsilon, \tau}^{1}} \leq \sqrt{5 L}\left(E_{1 ; \epsilon, \tau}(t) e^{-\frac{\lambda L}{\epsilon \sqrt{\tau}}}+E_{2 ; \epsilon, \tau}(t) e^{-\frac{\lambda\left(L-L_{0}\right)}{\epsilon \sqrt{\tau}}}\right)$. 
Proof. Because of the definition of $u_{L}$ and $v_{L}$ given in 2.22) and 2.25), Lemmas 2.3.4(iii) and inequality (2.38), we have that

$$
\begin{aligned}
\left\|\left(u_{L}(\cdot, t)-v_{L}(\cdot, t)\right)_{x}\right\|_{\infty} & \leq\left|c_{1}(t)\right| e^{-\frac{L}{\epsilon \sqrt{\tau}}}\left|\phi_{2}^{\prime}(x)\right|+\left|U_{c_{2}}(L, t)\right|\left|\phi_{2}^{\prime}(x)\right| \\
& \leq \frac{2}{\epsilon \sqrt{\tau}}\left(E_{1 ; \epsilon, \tau}(t) e^{-\frac{\lambda L}{\epsilon \sqrt{\tau}}}+E_{2 ; \epsilon, \tau}(t) e^{-\frac{\lambda\left(L-L_{0}\right)}{\epsilon \sqrt{\tau}}}\right) .
\end{aligned}
$$

Now, combining (2.39) and 2.40), we obtain that

$$
\begin{aligned}
\left\|u_{L}(\cdot, t)-v_{L}(\cdot, t)\right\|_{H_{L, \epsilon, \tau}^{1}} & =\sqrt{\int_{0}^{L}\left|u_{L}-v_{L}\right|^{2}+\left|\epsilon \sqrt{\tau}\left(u_{L}-v_{L}\right)_{x}\right|^{2} d x} \\
& \leq \sqrt{5 L}\left(E_{1 ; \epsilon, \tau}(t) e^{-\frac{\lambda L}{\epsilon \sqrt{\tau}}}+E_{2 ; \epsilon, \tau}(t) e^{-\frac{\lambda\left(L-L_{0}\right)}{\epsilon \sqrt{\tau}}}\right) .
\end{aligned}
$$

Proposition 2.3.7. If $u_{0}(x)$ satisfies 2.30, then

$$
\|W(\cdot, t)\|_{H_{L, \epsilon, \tau}^{1}} \leq \gamma_{1 ; \epsilon, \tau}(t) e^{-\frac{\lambda L}{\epsilon \sqrt{ } \tau}}+\gamma_{2 ; \epsilon, \tau}(t) e^{-\frac{\lambda\left(L-L_{0}\right)}{\epsilon \sqrt{\tau}}}
$$

where the coefficients $\gamma_{1 ; \epsilon, \tau}(t)$ and $\gamma_{2 ; \epsilon, \tau}(t)$ are derived as

$$
\begin{aligned}
& \gamma_{1 ; \epsilon, \tau}(t)=e^{\frac{(M+1)^{2} t}{2 M \epsilon \sqrt{\tau}}}\left(\frac{(M+1)^{2} \sqrt{\tau}}{2 M}+1\right) \sqrt{L}\left(\frac{t}{\epsilon \tau}\left|c_{1}(\cdot)\right|_{\infty}+\frac{a_{\tau}}{b_{\tau}}\left(e^{\frac{b_{\tau} t}{\epsilon \tau}}-1\right)\right), \\
& \gamma_{2 ; \epsilon, \tau}(t)=e^{\frac{(M+1)^{2} t}{2 M \epsilon \sqrt{\tau}}}\left(\frac{(M+1)^{2} \sqrt{\tau}}{2 M}+1\right) \sqrt{L} c_{\tau}\left(\frac{t}{\epsilon \tau\left(b_{\tau}-1\right)} e^{\frac{\left(b_{\tau}-1\right) t}{\epsilon \tau}}-\frac{1}{\left(b_{\tau}-1\right)^{2}}\left(e^{\frac{\left(b_{\tau}-1\right) t}{\epsilon \tau}}-1\right)\right) .
\end{aligned}
$$

Proof. Multiplying the governing equation of $W(2.28)$ by $2 W$, integrating over $[0, L]$,

$$
\begin{aligned}
& \int_{0}^{L} 2 W W_{t} d x-\epsilon \int_{0}^{L} 2 W W_{x x} d x-\int_{0}^{L} \epsilon^{2} \tau 2 W W_{x x t} d x \\
= & -\int_{0}^{t} 2 W(f(v)-f(u))_{x} d x+\frac{1}{\epsilon \tau} \int_{0}^{L} 2 W\left(v_{L}-u_{L}\right) d x,
\end{aligned}
$$


and using integration by parts, we get

$$
\begin{aligned}
& \frac{d}{d t} \int_{0}^{L} W^{2}+\left(\epsilon \sqrt{\tau} W_{x}\right)^{2} d x \\
= & -\epsilon \int_{0}^{L} 2 W_{x}^{2} d x+\int_{0}^{L} 2 W_{x}(f(v)-f(u)) d x+\frac{2}{\epsilon \tau} \int_{0}^{L} W\left(v_{L}-u_{L}\right) d x .
\end{aligned}
$$

Therefore, using the norm we defined earlier in 2.31), we have that

$$
\begin{aligned}
& \frac{d}{d t}\|W(\cdot, t)\|_{H_{L, \epsilon, \tau}^{1}}^{2} \\
\leq & 2 \int_{0}^{L}\left|W_{x}\left\|f^{\prime}(\eta)\right\| v-u\right| d x+\frac{2 \sqrt{L}}{\epsilon \mathcal{T}}\left\|v_{L}-u_{L}\right\|_{\infty}\|W(\cdot, t)\|_{H_{L, \epsilon, \tau}^{1}}
\end{aligned}
$$

and notice that

$$
f^{\prime}(u) \leq \frac{(M+1)^{2}}{2 M}
$$

we denote this upper bound by $C$, i.e.,

$$
C=\frac{(M+1)^{2}}{2 M},
$$

then we have that

$$
\begin{aligned}
& \frac{d}{d t}\|W(\cdot, t)\|_{H_{L, \epsilon, \tau}^{1}}^{2} \\
\leq & 2 C \int_{0}^{L}\left|W_{x}\right|\left(|W|+\left\|v_{L}-u_{L}\right\|_{\infty}\right) d x+\frac{2 \sqrt{L}}{\epsilon \tau}\left\|v_{L}-u_{L}\right\|_{\infty}\|W(\cdot, t)\|_{H_{L, \epsilon, \tau}^{1}} \\
\leq & \frac{2 C}{\epsilon \sqrt{\tau}}\left(\|W(\cdot, t)\|_{H_{L, \epsilon, \tau}^{1}}^{2}+\left\|v_{L}-u_{L}\right\|_{\infty} \sqrt{L}\|W(\cdot, t)\|_{H_{L, \epsilon, \tau}^{1}}\right) \\
& \quad+\frac{2 \sqrt{L}}{\epsilon \tau}\left\|v_{L}-u_{L}\right\|_{\infty}\|W(\cdot, t)\|_{H_{L, \epsilon, \tau}^{1}} \\
= & \frac{2 C}{\epsilon \sqrt{\tau}}\|W(\cdot, t)\|_{H_{L, \epsilon, \tau}^{1}}^{2}+\left(\frac{2 C}{\epsilon \sqrt{\tau}}+\frac{2}{\epsilon \tau}\right) \sqrt{L}\left\|v_{L}-u_{L}\right\|_{\infty}\|W(\cdot, t)\|_{H_{L, \epsilon, \tau}^{1}} .
\end{aligned}
$$


Hence,

$$
\frac{d}{d t}\|W(\cdot, t)\|_{H_{L, \epsilon, \tau}^{1}} \leq \frac{C}{\epsilon \sqrt{\tau}}\|W(\cdot, t)\|_{H_{L, \epsilon, \tau}^{1}}+\left(\frac{C}{\epsilon \sqrt{\tau}}+\frac{1}{\epsilon \tau}\right) \sqrt{L}\left\|v_{L}-u_{L}\right\|_{\infty} .
$$

By Gronwall's inequality and 2.39

$$
\begin{aligned}
\|W(\cdot, t)\|_{H_{L, \epsilon, \tau}^{1} \leq} \leq & \int_{0}^{t}\left(\frac{C}{\epsilon \sqrt{\tau}}+\frac{1}{\epsilon \tau}\right) \sqrt{L}\left\|v_{L}-u_{L}\right\|_{\infty} e^{\frac{C(t-s)}{\epsilon \sqrt{\tau}}} d s \\
\leq & e^{\frac{C t}{\epsilon \sqrt{\tau}}}\left(\frac{C}{\epsilon \sqrt{\tau}}+\frac{1}{\epsilon \tau}\right) \sqrt{L} \int_{0}^{t} E_{1 ; \epsilon, \tau}(s) e^{-\frac{\lambda L}{\epsilon \sqrt{\tau}}}+E_{2 ; \epsilon, \tau}(s) e^{-\frac{\lambda\left(L-L_{0}\right)}{\epsilon \sqrt{\tau}}} d s \\
\leq & \left(e^{\frac{C t}{\epsilon \sqrt{\tau}}}\left(\frac{C}{\epsilon \sqrt{\tau}}+\frac{1}{\epsilon \tau}\right) \sqrt{L} \int_{0}^{t} E_{1 ; \epsilon, \tau}(s) d s\right) e^{-\frac{\lambda L}{\epsilon \sqrt{\tau}}} \\
& +\left(e^{\frac{C t}{\epsilon \sqrt{\tau}}}\left(\frac{C}{\epsilon \sqrt{\tau}}+\frac{1}{\epsilon \tau}\right) \sqrt{L} \int_{0}^{t} E_{2 ; \epsilon, \tau}(s) d s\right) e^{-\frac{\lambda\left(L-L_{0}\right)}{\epsilon \sqrt{\tau}}} \\
\leq & e^{\frac{C t}{\epsilon \sqrt{\tau}}}\left(\frac{C}{\epsilon \sqrt{\tau}}+\frac{1}{\epsilon \tau}\right) \sqrt{L}\left(t\left|c_{1}(\cdot)\right|_{\infty}+\frac{a_{\tau} \epsilon \tau}{b_{\tau}}\left(e^{\frac{b \tau t}{\epsilon \tau}}-1\right)\right) e^{-\frac{\lambda L}{\epsilon \sqrt{\tau}}} \\
& +e^{\frac{C t}{\epsilon \sqrt{\tau}}}\left(\frac{C}{\epsilon \sqrt{\tau}}+\frac{1}{\epsilon \tau}\right) \sqrt{L} \frac{c_{\tau}}{\epsilon \tau} \\
& \cdot\left(\frac{\epsilon \tau}{b_{\tau}-1} t e^{\frac{(b \tau-1) t}{\epsilon \tau}}-\left(\frac{\epsilon \tau}{b_{\tau}-1}\right)^{2}\left(e^{\frac{(b \tau-1) t}{\epsilon \tau}}-1\right)\right) e^{-\frac{\lambda\left(L-L_{0}\right)}{\epsilon \sqrt{\tau}}} .
\end{aligned}
$$

Hence

$$
\|W(\cdot, t)\|_{H_{L, \epsilon, \tau}^{1}} \leq \gamma_{1 ; \epsilon, \tau}(t) e^{-\frac{\lambda L}{\epsilon \sqrt{\tau}}}+\gamma_{2 ; \epsilon, \tau}(t) e^{-\frac{\lambda\left(L-L_{0}\right)}{\epsilon \sqrt{\tau}}}
$$

where

$$
\begin{aligned}
& \gamma_{1 ; \epsilon, \tau}(t)=e^{\frac{C t}{\epsilon \sqrt{\tau}}}(C \sqrt{\tau}+1) \sqrt{L}\left(\frac{t}{\epsilon \tau}\left|c_{1}(\cdot)\right|_{\infty}+\frac{a_{\tau}}{b_{\tau}}\left(e^{\frac{b_{\tau} t}{\epsilon \tau}}-1\right)\right), \\
& \gamma_{2 ; \epsilon, \tau}(t)=e^{\frac{C t}{\epsilon \sqrt{\tau}}}(C \sqrt{\tau}+1) \sqrt{L} c_{\tau}\left(\frac{t}{\epsilon \tau\left(b_{\tau}-1\right)} e^{\frac{\left(b_{\tau}-1\right) t}{\epsilon \tau}}-\frac{1}{\left(b_{\tau}-1\right)^{2}}\left(e^{\frac{\left(b_{\tau}-1\right) t}{\epsilon \tau}}-1\right)\right) .
\end{aligned}
$$

Now comes the main theorem of this section. 
Theorem 2.3.8. If $u_{0}(x)$ satisfies (2.30), and $E_{1 ; \epsilon, \tau}(t), E_{2 ; \epsilon, \tau}(t), \gamma_{1 ; \epsilon, \tau}(t), \gamma_{2 ; \epsilon, \tau}(t)$ are as in proposition 2.3.5 and 2.3.7, then $\|u(\cdot, t)-v(\cdot, t)\|_{H_{L, \epsilon, \tau}^{1}} \leq D_{1 ; \epsilon, \tau}(t) e^{-\frac{\lambda L}{\epsilon \sqrt{\tau}}}+$ $D_{2 ; \epsilon, \tau}(t) e^{-\frac{\lambda\left(L-L_{0}\right)}{\epsilon \sqrt{\tau}}}$ where $D_{1 ; \epsilon, \tau}(t)=\gamma_{1 ; \epsilon, \tau}(t)+\sqrt{5 L} E_{1 ; \epsilon, \tau}(t)$ and $D_{2 ; \epsilon, \tau}(t)=\gamma_{2 ; \epsilon, \tau}(t)+$ $\sqrt{5 L} E_{2 ; \epsilon, \tau}(t)$.

Proof of the Main Theorem.

$$
\begin{aligned}
\|u(\cdot, t)-v(\cdot, t)\|_{H_{L, \epsilon, \tau}^{1}} & \leq\|W(\cdot, t)\|_{H_{L, \epsilon, \tau}^{1}}+\left\|v_{L}(\cdot, t)-u_{L}(\cdot, t)\right\|_{H_{L, \epsilon, \tau}^{1}} \\
& =D_{1 ; \epsilon, \tau}(t) e^{-\frac{\lambda L}{\epsilon \sqrt{\tau}}}+D_{2 ; \epsilon, \tau}(t) e^{-\frac{\lambda\left(L-L_{0}\right)}{\epsilon \sqrt{\tau}}}
\end{aligned}
$$

where

$$
\begin{aligned}
D_{1 ; \epsilon, \tau}(t)= & \gamma_{1 ; \epsilon, \tau}(t)+\sqrt{5 L} E_{1 ; \epsilon, \tau}(t) \\
= & e^{\frac{C t}{\epsilon \sqrt{\tau}}}(C \sqrt{\tau}+1) \sqrt{L}\left(\frac{t}{\epsilon \tau}\left|c_{1}(\cdot)\right|_{\infty}+\frac{a_{\tau}}{b_{\tau}}\left(e^{\frac{b_{\tau} t}{\epsilon \tau}}-1\right)\right) \\
& +\sqrt{5 L}\left(|c(\cdot)|_{\infty}+a_{\tau} e^{\frac{b_{\tau} t}{\epsilon \tau}}\right), \\
D_{2 ; \epsilon, \tau}(t)= & \gamma_{2 ; \epsilon, \tau}(t)+\sqrt{5 L} E_{2 ; \epsilon, \tau}(t) \\
= & e^{\frac{C t}{\epsilon \sqrt{\tau}}}(C \sqrt{\tau}+1) \sqrt{L} c_{\tau}\left(\frac{t}{\epsilon \tau\left(b_{\tau}-1\right)} e^{\frac{\left(b_{\tau}-1\right) t}{\epsilon \tau}}-\frac{1}{\left(b_{\tau}-1\right)^{2}}\left(e^{\frac{\left(b_{\tau}-1\right) t}{\epsilon \tau}}-1\right)\right) \\
& +\sqrt{5 L} c_{\tau} \frac{t}{\epsilon \tau} e^{\frac{\left(b_{\tau}-1\right) t}{\epsilon \tau}} .
\end{aligned}
$$

This theorem shows that if $\frac{\lambda L}{\epsilon \sqrt{\tau}}$ and $\frac{\lambda\left(L-L_{0}\right)}{\epsilon \sqrt{\tau}}$ converge to infinity, then the solution $v(x, t)$ of the finite interval boundary value problem converges to the solution $u(x, t)$ of the half line problem in the sense of $\|\cdot\|_{H_{L, \epsilon, \tau}^{1}}$. This can be achieved either by letting $L \rightarrow \infty$ or $\epsilon \rightarrow 0$. For example, in the extreme case, $\epsilon=0$, the half line problem (2.2) becomes hyperbolic and the domain of dependence is finite, so, certainly, one 
only need to consider the finite interval boundary value problem. This is consistent with the main theorem in the sense that for a fixed final time $t$, if $\lambda L>b_{\tau} t$ and $\lambda\left(L-L_{0}\right)>\left(b_{\tau}-1\right) t$, i.e., $L>\max \left(\frac{b_{\tau} t}{\lambda}, \frac{\left(b_{\tau}-1\right) t}{\lambda}\right)$, then $\|u(\cdot, t)-v(\cdot, t)\|_{H_{L, \epsilon, \tau}^{1}} \leq$ $D_{1 ; \epsilon, \tau}(t) e^{-\frac{\lambda L}{\epsilon \sqrt{\tau}}}+D_{2 ; \epsilon, \tau}(t) e^{-\frac{\lambda\left(L-L_{0}\right)}{\epsilon \sqrt{\tau}}} \rightarrow 0$ as $\epsilon \rightarrow 0$. Theorem 2.3.8 gives a theoretical justification for using the solution of the finite interval boundary value problem to approximate the solution of the half line problem with appropriate choice of $L$ and $\epsilon$. Hence in the next chapter, the numerical scheme designed to solve the MBL equation (1.19) is given for finite interval boundary value problem. 


\section{CHAPTER 3}

\section{NUMERICAL SCHEMES}

\subsection{Second-Order Schemes}

In this section, we show how to apply the central schemes [17] originally designed for hyperbolic conservation laws to numerically solve the MBL equation (1.19), which is of pseudo-parabolic type. We first collect all the terms with time derivative and rewrite MBL equation (1.19) as

$$
\left(u-\epsilon^{2} \tau u_{x x}\right)_{t}+(f(u))_{x}=\epsilon u_{x x}
$$

By letting

$$
w=u-\epsilon^{2} \tau u_{x x} \Longleftrightarrow u=\left(I-\epsilon^{2} \tau \partial_{x x}\right)^{-1} w
$$

MBL equation (3.1) can be written as

$$
w_{t}+(f(u))_{x}=\epsilon u_{x x}
$$

Now, the new form of MBL equation (3.3) can be viewed as a PDE in terms of $w$,

and the occurrence of $u$ can be recovered by 3.2 . Equation 3.3 can be formally viewed as

$$
w_{t}+\left(f\left(\left(I-\epsilon^{2} \tau \partial_{x x}\right)^{-1} w\right)\right)_{x}=\epsilon\left(\left(I-\epsilon^{2} \tau \partial_{x x}\right)^{-1} w\right)_{x x}
$$


which is a balance law in term of $w$. Hence to solve (3.3), we modify the central scheme [17]. As in [17], at each time level, we first reconstruct a piecewise linear approximation of the form

$$
L_{j}(x, t)=w_{j}(t)+\left(x-x_{j}\right) \frac{w_{j}^{\prime}}{\Delta x} \quad x_{j-\frac{1}{2}} \leq x \leq x_{j+\frac{1}{2}} .
$$

Second-order accuracy is guaranteed if the so-called vector of numerical derivative $\frac{w_{j}^{\prime}}{\Delta x}$, which will be given later, satisfies

$$
\frac{w_{j}^{\prime}}{\Delta x}=\frac{\partial w\left(x_{j}, t\right)}{\partial x}+O(\Delta x) .
$$

We denote the staggered piecewise-constant functions $\bar{w}_{j+\frac{1}{2}}(t)$ as

$$
\bar{w}_{j+\frac{1}{2}}(t)=\frac{1}{\Delta x} \int_{x_{j}}^{x_{j+1}} w(x, t) d x .
$$

Evolve the piecewise linear interplant (3.5) by integrating (3.3) over $\left[x_{j}, x_{j+1}\right] \times[t, t+$ $\Delta t]$, we get that

$$
\begin{aligned}
\bar{w}_{j+\frac{1}{2}}(t+\Delta t)= & \bar{w}_{j+\frac{1}{2}}(t) \\
& -\frac{1}{\Delta x}\left[\int_{t}^{t+\Delta t} f\left(u\left(x_{j+1}, s\right)\right) d s-\int_{t}^{t+\Delta t} f\left(u\left(x_{j}, s\right)\right) d s\right] \\
& +\frac{\epsilon}{\Delta x}\left[\int_{t}^{t+\Delta t} \int_{x_{j}}^{x_{j+1}} \frac{\partial^{2} u(x, s)}{\partial x^{2}} d x d s\right] .
\end{aligned}
$$

Using the definition of $L_{j}(x, t)$ and $L_{j+1}(x, t)$ given by (3.5), we have that

$$
\begin{aligned}
\frac{1}{\Delta x} \int_{x_{j}}^{x_{j+\frac{1}{2}}} L_{j}(x, t) d x & =\frac{1}{\Delta x} \int_{x_{j}}^{x_{j+\frac{1}{2}}} w_{j}(t)+\left(x-x_{j}\right) \frac{w_{j}^{\prime}}{\Delta x} d x \\
& =\frac{1}{2} w_{j}(t)+\frac{1}{8} w_{j}^{\prime}, \\
\frac{1}{\Delta x} \int_{x_{j+\frac{1}{2}}}^{x_{j+1}} L_{j+1}(x, t) d x & =\frac{1}{\Delta x} \int_{x_{j+\frac{1}{2}}}^{x_{j+1}} w_{j+1}(t)+\left(x-x_{j+1}\right) \frac{w_{j+1}^{\prime}}{\Delta x} d x \\
& =\frac{1}{2} w_{j+1}(t)-\frac{1}{8} w_{j+1}^{\prime},
\end{aligned}
$$


and hence

$$
\begin{aligned}
\bar{w}_{j+\frac{1}{2}}(t) & =\frac{1}{\Delta x} \int_{x_{j}}^{x_{j+\frac{1}{2}}} L_{j}(x, t) d x+\frac{1}{\Delta x} \int_{x_{j+\frac{1}{2}}}^{x_{j+1}} L_{j+1}(x, t) d x \\
& =\frac{1}{2}\left(w_{j}(t)+w_{j+1}(t)\right)+\frac{1}{8}\left(w_{j}^{\prime}-w_{j+1}^{\prime}\right) .
\end{aligned}
$$

Moreover, if the CFL condition

$$
\begin{aligned}
& \lambda \cdot \max _{x_{j} \leq x \leq x_{j+1}} \rho(A(w(x, t)))<\frac{1}{2} \\
& \text { where } \quad \lambda=\frac{\Delta t}{\Delta x}, \quad A=\frac{\partial f}{\partial w} \quad \text { and } \quad \rho(A)=\text { spectrum radius of A }
\end{aligned}
$$

is met, then the middle two integrands can be approximated by the midpoint rule

$$
\begin{aligned}
\int_{t}^{t+\Delta t} f\left(u\left(x_{j}, s\right)\right) d s & =f\left(u\left(x_{j}, t+\frac{\Delta t}{2}\right)\right) \Delta t+O\left(\Delta t^{3}\right) \\
\int_{t}^{t+\Delta t} f\left(u\left(x_{j+1}, s\right)\right) d s & =f\left(u\left(x_{j+1}, t+\frac{\Delta t}{2}\right)\right) \Delta t+O\left(\Delta t^{3}\right) .
\end{aligned}
$$

Combining (3.8)-(3.11), we obtain

$$
\begin{aligned}
\bar{w}_{j+\frac{1}{2}}(t+\Delta t)= & \frac{1}{2}\left[w_{j}(t)+w_{j+1}(t)\right]+\frac{1}{8}\left[w_{j}^{\prime}-w_{j+1}^{\prime}\right] \\
& -\lambda\left[f\left(u_{j+1}\left(t+\frac{\Delta t}{2}\right)-f\left(u_{j}\left(t+\frac{\Delta t}{2}\right)\right)\right]\right. \\
& +\frac{\epsilon}{\Delta x}\left[\int_{t}^{t+\Delta t} \int_{x_{j}}^{x_{j+1}} \frac{\partial^{2} u(x, s)}{\partial x^{2}} d x d s\right] .
\end{aligned}
$$

Nessyahu and Tadmor in [17] have introduced many ways to estimate the derivatives, so, we won't reproduce them here. Instead, we will focus on the last integral in (3.12). There are many ways to numerically calculate the integral $\int_{t}^{t+\Delta t} \int_{x_{j}}^{x_{j+1}} \frac{\partial^{2} u(x, s)}{\partial x^{2}} d x d s$. We will show two ways to do this in the following two subsections. Both of them achieve second order accuracy. 


\subsubsection{Trapezoid Scheme}

In this scheme, we use the notion (3.7), the definition $(3.2)$ and the trapezoid rule to calculate the integral numerically as follows:

$$
\begin{aligned}
& \int_{t}^{t+\Delta t} \int_{x_{j}}^{x_{j+1}} \frac{\partial^{2} u(x, s)}{\partial x^{2}} d x d s \\
= & \Delta x \int_{t}^{t+\Delta t}\left(\bar{u}_{x x}\right)_{j+\frac{1}{2}}(s) d s \\
= & \left.\frac{\Delta x \Delta t}{2}\left(\left(\bar{u}_{x x}\right)_{j+\frac{1}{2}}(t)+\left(\bar{u}_{x x}\right)_{j+\frac{1}{2}}(t+\Delta t)\right)\right)
\end{aligned}
$$

with $\mathcal{O}\left(\Delta t^{3}\right)$ error. Combining with 3.12 , we can get

$$
\begin{aligned}
& \left(I-\epsilon^{2} \tau \partial_{x x}\right) \bar{u}_{j+\frac{1}{2}}(t+\Delta t) \\
= & \bar{w}_{j+\frac{1}{2}}(t)-\lambda\left[f\left(u_{j+1}\left(t+\frac{\Delta t}{2}\right)\right)-f\left(u_{j}\left(t+\frac{\Delta t}{2}\right)\right)\right] \\
& +\frac{\epsilon \Delta t}{2}\left(\left(\bar{u}_{x x}\right)_{j+\frac{1}{2}}(t)+\left(\bar{u}_{x x}\right)_{j+\frac{1}{2}}(t+\Delta t)\right) .
\end{aligned}
$$

Using the cell average of $w$ given in 3.10 , we have

$$
\begin{aligned}
& \left(I-\left(\epsilon^{2} \tau+\frac{\epsilon \Delta t}{2}\right) \partial_{x x}\right) \bar{u}_{j+\frac{1}{2}}(t+\Delta t) \\
= & \left(I-\left(\epsilon^{2} \tau-\frac{\epsilon \Delta t}{2}\right) \partial_{x x}\right) \bar{u}_{j+\frac{1}{2}}(t) \\
& -\lambda\left[f\left(u_{j+1}\left(t+\frac{\Delta t}{2}\right)\right)-f\left(u_{j}\left(t+\frac{\Delta t}{2}\right)\right)\right] .
\end{aligned}
$$

The flow char of the trapezoid scheme is given in table 3.1 . 


\section{Trapezoid Scheme}

Calculate $\quad \bar{w}_{j+\frac{1}{2}}(t)=\frac{1}{2}\left(w_{j}(t)+w_{j+1}(t)\right)+\frac{1}{8}\left(w_{j}^{\prime}-w_{j+1}^{\prime}\right)$

Solve $\left(I-\epsilon^{2} \tau \partial_{x x}\right) \bar{u}_{j+\frac{1}{2}}(t)=\bar{w}_{j+\frac{1}{2}}(t)$ for $\bar{u}_{j+\frac{1}{2}}(t)$

Calculate $w_{j}\left(t+\frac{\Delta t}{2}\right)=w_{j}(t)+\left(\epsilon \frac{\Delta^{2} u_{j}}{\Delta x}-f_{j}^{\prime}\right) \frac{\lambda}{2}$

Solve $\left(I-\epsilon^{2} \tau \partial_{x x}\right) u_{j}\left(t+\frac{\Delta t}{2}\right)=w_{j}\left(t+\frac{\Delta t}{2}\right)$ for $u_{j}\left(t+\frac{\Delta t}{2}\right)$

Solve $\quad\left(I-\left(\epsilon^{2} \tau+\frac{\epsilon \Delta t}{2}\right) \partial_{x x}\right) \bar{u}_{j+\frac{1}{2}}(t+\Delta t)$

$=\left(I-\left(\epsilon^{2} \tau-\frac{\epsilon \Delta t}{2}\right) \partial_{x x}\right) \bar{u}_{j+\frac{1}{2}}(t)$

$-\lambda\left[f\left(u_{j+1}\left(t+\frac{\Delta t}{2}\right)\right)-f\left(u_{j}\left(t+\frac{\Delta t}{2}\right)\right)\right] \quad$ for $\bar{u}_{j+\frac{1}{2}}(t+\Delta t)$

Table 3.1: Flow chart for Trapezoid Scheme 


\subsubsection{Midpoint Scheme}

In this scheme, we use the notion $(3.7)$, the definition 3.2 and the midpoint rule to calculate the integral numerically as follows:

$$
\begin{aligned}
\int_{t}^{t+\Delta t} \int_{x_{j}}^{x_{j+1}} \frac{\partial^{2} u(x, s)}{\partial x^{2}} d x d s & =\Delta x \int_{t}^{t+\Delta t}\left(\bar{u}_{x x}\right)_{j+\frac{1}{2}}(s) d s \\
& =\Delta x \Delta t\left(\bar{u}_{x x}\right)_{j+\frac{1}{2}}\left(t+\frac{\Delta t}{2}\right)
\end{aligned}
$$

Combining with 3.12 and the definition 3.2 , we get

$$
\begin{aligned}
\left(I-\epsilon^{2} \tau \partial_{x x}\right) \bar{u}_{j+\frac{1}{2}}(t+\Delta t)= & \frac{1}{2}\left[w_{j}(t)+w_{j+1}(t)\right]+\frac{1}{8}\left[w_{j}^{\prime}-w_{j+1}^{\prime}\right] \\
& -\lambda\left[f\left(u_{j+1}\left(t+\frac{\Delta t}{2}\right)-f\left(u_{j}\left(t+\frac{\Delta t}{2}\right)\right)\right]\right. \\
& +\epsilon \Delta t\left(\bar{u}_{x x}\right)_{j+\frac{1}{2}}\left(t+\frac{\Delta t}{2}\right) .
\end{aligned}
$$

The flow chart of the midpoint scheme is given in table 3.2 .

In both schemes 3.15 and 3.17 introduced so far, the $u\left(\cdot, t+\frac{\Delta t}{2}\right)$ 's are used. To estimate $u\left(\cdot, t+\frac{\Delta t}{2}\right)$ 's, we use Taylor expansion and the conservation law 3.3 :

$$
\begin{aligned}
w\left(x_{j}, t+\frac{\Delta t}{2}\right) & =w_{j}(t)+\frac{\partial w}{\partial t} \frac{\Delta t}{2}+\mathcal{O}\left(\Delta t^{2}\right) \\
& =w_{j}(t)+\left(\epsilon \frac{\partial^{2} u}{\partial x^{2}}-\frac{\partial f}{\partial x}\right) \frac{\Delta t}{2}+\mathcal{O}\left(\Delta t^{2}\right) \\
& =w_{j}(t)+\left(\epsilon \frac{\Delta^{2} u_{j}}{\Delta x}-f_{j}^{\prime}\right) \frac{\lambda}{2}
\end{aligned}
$$

and the second-order accuracy is met if

$$
\frac{f_{j}^{\prime}}{\Delta x}=\frac{\partial f\left(u\left(x_{j}, t\right)\right)}{\partial x}+\mathcal{O}(\Delta x) .
$$




\section{Midpoint Scheme}

$$
\begin{aligned}
& \text { Calculate } \quad \bar{w}_{j+\frac{1}{2}}(t)=\frac{1}{2}\left(w_{j}(t)+w_{j+1}(t)\right)+\frac{1}{8}\left(w_{j}^{\prime}-w_{j+1}^{\prime}\right) \\
& \text { Calculate } \quad w_{j}\left(t+\frac{\Delta t}{2}\right)=w_{j}(t)+\left(\epsilon \frac{\Delta^{2} u_{j}}{\Delta x}-f_{j}^{\prime}\right) \frac{\lambda}{2} \\
& \text { Solve }\left(I-\epsilon^{2} \tau \partial_{x x}\right) u_{j}\left(t+\frac{\Delta t}{2}\right)=w_{j}\left(t+\frac{\Delta t}{2}\right) \text { for } u_{j}\left(t+\frac{\Delta t}{2}\right) \\
& \text { Calculate } \quad \bar{w}_{j+\frac{1}{2}}\left(t+\frac{\Delta t}{2}\right)=\frac{1}{2}\left(w_{j}\left(t+\frac{\Delta t}{2}\right)+w_{j+1}\left(t+\frac{\Delta t}{2}\right)\right) \\
& +\frac{1}{8}\left(w_{j}^{\prime}\left(t+\frac{\Delta t}{2}\right)-w_{j+1}^{\prime}\left(t+\frac{\Delta t}{2}\right)\right) \\
& \text { Solve }\left(I-\epsilon^{2} \tau \partial_{x x}\right) \bar{u}_{j+\frac{1}{2}}\left(t+\frac{\Delta t}{2}\right)=\bar{w}_{j+\frac{1}{2}}\left(t+\frac{\Delta t}{2}\right) \text { for } \bar{u}_{j+\frac{1}{2}}\left(t+\frac{\Delta t}{2}\right) \\
& \text { Solve } \quad\left(I-\epsilon^{2} \tau \partial_{x x}\right) \bar{u}_{j+\frac{1}{2}}(t+\Delta t) \\
& =\frac{1}{2}\left[w_{j}(t)+w_{j+1}(t)\right]+\frac{1}{8}\left[w_{j}^{\prime}-w_{j+1}^{\prime}\right] \\
& -\lambda\left[f\left(u_{j+1}\left(t+\frac{\Delta t}{2}\right)-f\left(u_{j}\left(t+\frac{\Delta t}{2}\right)\right)\right]\right. \\
& +\epsilon \Delta t\left(\bar{u}_{x x}\right)_{j+\frac{1}{2}}\left(t+\frac{\Delta t}{2}\right) \text { for } \bar{u}_{j+\frac{1}{2}}(t+\Delta t)
\end{aligned}
$$

Table 3.2: Flow chart for Midpoint Scheme 
The choices for $\left\{v_{j}^{\prime}\right\}$ and $\left\{f_{j}^{\prime}\right\}$ can be found in [17], we used

$$
w_{j}^{\prime}=M M\left\{\Delta w_{j+\frac{1}{2}}, \Delta w_{j-\frac{1}{2}}\right\}, \quad f_{j}^{\prime}=M M\left\{\Delta f_{j+\frac{1}{2}}, \Delta f_{j-\frac{1}{2}}\right\}
$$

where $M M\{x, y\}=\operatorname{minmod}(x, y)=\frac{1}{2}(\operatorname{sgn}(x)+\operatorname{sgn}(y)) \cdot \operatorname{Min}(|x|,|y|)$ and $\Delta w_{j+\frac{1}{2}}=$ $w_{j+1}-w_{j}$.

Notice that for both schemes, one needs to solve elliptic equation $\left(I-a \partial_{x x}\right) u=$ r.h.s three times for one step time advance. The flow charts for the Trapezoid scheme and Midpoint scheme are given in Tables 3.1 and 3.2 .

\subsection{A Third Order Semi-discrete Scheme}

Similarly, we can extend the third order scheme to solve MBL equation (1.19), however, it is more involved. But the third order semi-discrete central scheme proposed in [12] can be extended to solve the MBL equation in a straightforward manner. In order to make the paper self-contained, we include the formulation below.

$$
\frac{d \bar{w}_{j}}{d t}=-\frac{H_{j+1 / 2}(t)-H_{j-1 / 2}(t)}{\Delta x}+\epsilon Q_{j}(t)
$$

where $\bar{w}(x, t)$ denotes the cell average of $w$

$$
\bar{w}_{j}(t)=\frac{1}{\Delta x} \int_{x_{j-1 / 2}}^{x_{j+1 / 2}} w(x, t) d x,
$$

$H_{j+1 / 2}(t)$ is the numerical convection flux and $Q_{j}(t)$ is a high-order approximation to the diffusion term $u_{x x}$.

$$
H_{j+1 / 2}(t)=\frac{f\left(u_{j+1 / 2}^{+}(t)\right)+f\left(u_{j+1 / 2}^{-}(t)\right)}{2}-\frac{a_{j+1 / 2}(t)}{2}\left[w_{j+1 / 2}^{+}(t)-w_{j+1 / 2}^{-}(t)\right],
$$


where $u_{j+1 / 2}^{-}(t), u_{j+1 / 2}^{+}(t)$ denote the left and right intermediate values of $u\left(x, t^{n}\right)$ at $x_{j+1 / 2}$, and their values are converted from the $w_{j+1 / 2}^{-}(t), w_{j+1 / 2}^{+}(t)$ using 3.2 . The way to calculate $w_{j+1 / 2}^{-}(t), w_{j+1 / 2}^{+}(t)$ and $a_{j+1 / 2}(t)$ is

$$
\begin{aligned}
w_{j+1 / 2}^{+}(t) & =A_{j+1}-\frac{\Delta x}{2} B_{j+1}+\frac{(\Delta x)^{2}}{8} C_{j+1}, \\
w_{j+1 / 2}^{-}(t) & =A_{j}+\frac{\Delta x}{2} B_{j}+\frac{(\Delta x)^{2}}{8} C_{j}, \\
a_{j+1 / 2}(t) & =\max \left\{\frac{\partial f}{\partial u}\left(u_{j+1 / 2}^{-}(t)\right), \frac{\partial f}{\partial u}\left(u_{j+1 / 2}^{+}(t)\right)\right\},
\end{aligned}
$$

where

$$
\begin{aligned}
A_{j} & =\bar{w}_{j}^{n}-\frac{w_{C}}{12}\left(\bar{w}_{j+1}^{n}-2 \bar{w}_{j}^{n}+\bar{w}_{j-1}^{n}\right), \\
B_{j} & =\frac{1}{\Delta x}\left[w_{R}\left(\bar{w}_{j+1}^{n}-\bar{w}_{j}^{n}\right)+w_{C} \frac{\bar{w}_{j+1}^{n}-\bar{w}_{j-1}^{n}}{2}+w_{L}\left(\bar{w}_{j}^{n}-\bar{w}_{j-1}^{n}\right)\right], \\
C_{j} & =2 w_{C} \frac{\bar{w}_{j-1}^{n}-2 \bar{w}_{j}^{n}+\bar{w}_{j+1}^{n}}{\Delta x^{2}}, \\
w_{i} & =\frac{\alpha_{i}}{\sum_{m} \alpha_{m}} \quad \alpha_{i}=\frac{c_{i}}{\left(\epsilon_{0}+I S_{i}\right)^{p}}, \quad i, m \in\{C, R, L\} \\
c_{L} & =c_{R}=1 / 4, \quad c_{C}=1 / 2, \quad \epsilon_{0}=10^{-6}, \quad p=2, \\
I S_{L} & =\left(\bar{w}_{j}^{n}-\bar{w}_{j-1}^{n}\right)^{2}, \quad I S_{R}=\left(\bar{w}_{j+1}^{n}-\bar{w}_{j}^{n}\right)^{2}, \\
I S_{C} & =\frac{13}{3}\left(\bar{w}_{j+1}^{n}-2 \bar{w}_{j}^{n}+\bar{w}_{j-1}^{n}\right)^{2}+\frac{1}{4}\left(\bar{w}_{j+1}^{n}-\bar{w}_{j-1}^{n}\right)^{2} .
\end{aligned}
$$

The diffusion $u_{x x}$ is approximated using the following fourth-order central differencing form

$$
Q_{j}(t)=\frac{1}{12 \Delta x}\left[-\left(u_{x}\right)_{j+2, j}+8\left(u_{x}\right)_{j+1, j}-8\left(u_{x}\right)_{j-1, j}+\left(u_{x}\right)_{j-2, j}\right]
$$


where

$$
\begin{aligned}
\left(u_{x}\right)_{j+2, j} & =\frac{1}{12 \Delta x}\left[25 u_{j+2}(t)-48 u_{j+1}(t)+36 u_{j}(t)-16 u_{j-1}(t)+3 u_{j-2}(t)\right], \\
\left(u_{x}\right)_{j+1, j} & =\frac{1}{12 \Delta x}\left[3 u_{j+2}(t)+10 u_{j+1}(t)-18 u_{j}(t)+6 u_{j-1}(t)-u_{j-2}(t)\right] \\
\left(u_{x}\right)_{j-1, j} & =\frac{1}{12 \Delta x}\left[u_{j+2}(t)-6 u_{j+1}(t)+18 u_{j}(t)-10 u_{j-1}(t)-3 u_{j-2}(t)\right], \\
\left(u_{x}\right)_{j-2, j} & =\frac{1}{12 \Delta x}\left[-3 u_{j+2}(t)+16 u_{j+1}(t)-36 u_{j}(t)+48 u_{j-1}(t)-25 u_{j-2}(t)\right] .
\end{aligned}
$$

Notice that $\left\{u_{j}(t)\right\}$ can be recovered from $\left\{w_{j}(t)\right\}$ using 3.2 , and $\left\{w_{j}(t)\right\}$ are the point-values of the reconstructed polynomials

$$
w_{j}(t)=A_{j}+B_{j}\left(x-x_{j}\right)+\frac{1}{2} C_{j}\left(x-x_{j}\right)^{2} .
$$

The unique feature of this scheme is that the discretization is done in space first, and then the time evolution equation can be solved as a system of ordinary differential equations using any ODE solver of third order or higher. In this thesis, we simply use the standard fourth order Runge-Kutta methods. Notice that to achieve the third order accuracy, the linear solver that converts $u$ from $w$ using 3.2 need also to be high order. In this thesis, we use the following fourth order discretization of $\frac{\partial^{2} u}{\partial x^{2}}$

$$
\frac{\partial^{2} u}{\partial x^{2}}=\frac{-u_{j-2}+16 u_{j-1}-30 u_{j}+16 u_{j+1}-u_{j+2}}{12 \Delta x^{2}} .
$$

Hence to recover $u$ from $w$, we solve the following linear system

$$
A u=w
$$


where

$$
A=\left[\begin{array}{llllllllll}
c & d & e & & & & & & & \\
b & c & d & e & & & & & & \\
a & b & c & d & e & & & & & \\
& \ddots & \ddots & \ddots & \ddots & & & & & \\
& & \ddots & \ddots & \ddots & \ddots & & & & \\
& & & \ddots & \ddots & \ddots & \ddots & & & \\
& & & & \ddots & \ddots & \ddots & \ddots & & \\
& & & & & a & b & c & d & e \\
& & & & & & a & b & c & d \\
& & & & & & & a & b & c
\end{array}\right],
$$

$$
u=\left[\begin{array}{l}
u_{1} \\
u_{2} \\
\vdots \\
\vdots \\
u_{N-1} \\
u_{N}
\end{array}\right], \quad w=\left[\begin{array}{l}
w_{1}-b u_{B}-a u_{B} \\
w_{2}-a u_{B} \\
w_{3} \\
\vdots \\
w_{N-1}-e \cdot 0 \\
w_{N}-e \cdot 0-d \cdot 0
\end{array}\right] \text {, }
$$

and

$$
a=\frac{\epsilon^{2} \tau}{12 \Delta x^{2}}, \quad b=-\frac{16 \epsilon^{2} \tau}{12 \Delta x^{2}}, \quad c=1+\frac{30 \epsilon^{2} \tau}{12 \Delta x^{2}}, \quad d=-\frac{16 \epsilon^{2} \tau}{12 \Delta x^{2}}, \quad e=\frac{\epsilon^{2} \tau}{12 \Delta x^{2}}
$$




\section{CHAPTER 4 COMPUTATIONAL RESULTS}

In this chapter, we use the proposed second and third order schemes in chapter 3 to study the numerical solutions to the MBL equation

$$
u_{t}+(f(u))_{x}=\epsilon u_{x x}+\epsilon^{2} \tau u_{x x t}
$$

with the initial condition

$$
u_{0}(x)=\left\{\begin{array}{lll}
u_{B} & \text { if } & x=0 \\
0 & \text { if } & x>0
\end{array}\right.
$$

and the Dirichlet boundary condition.

In section 4.1, we will first test the accuracy of the schemes we will use to perform computation in the later sections. The solutions we will look for are the traveling-wave solutions to the MBL equation (4.1). For the completeness of the solution behavior, we will include Van Duijn et al [21] theorems on the existence of traveling-wave solution in section 4.2. Van Duijn et al [21] has shown that the solution will display qualitative different monotonicity behavior for different $u_{B}$ and $\tau$ values. Therefore, before performing the numerical computations, we provide the bifurcation diagram in section 4.3. Finally, in section 4.4, we will use examples to demonstrate qualitatively different numerical solution profiles. 


\subsection{Accuracy Analysis}

To validate the order analysis given in chapter 3 for various schemes proposed, we first test the order of our schemes numerically. To do this, we solve the MBL equation

$$
u_{t}+(f(u))_{x}=\epsilon u_{x x}+\epsilon^{2} \tau u_{x x t}
$$

and use a smooth initial condition

$$
u_{0}(x)=u_{B} H(x-5,5),
$$

where

$$
H(x, \xi)=\left\{\begin{array}{lll}
1 & \text { if } \quad x<-\xi \\
1-\frac{1}{2}\left(1+\frac{x}{\xi}+\frac{1}{\pi} \sin \left(\frac{\pi x}{\xi}\right)\right) & \text { if }-\xi \leq x \leq \xi \\
0 & \text { if } \quad x>\xi
\end{array}\right.
$$

and a stop time $T=1$ was employed, so that there was no shock created. $\epsilon$ in the MBL equation (4.1) is taken to be $1, M$ is taken to be 2 , and the computational interval is $[-10,20]$. The $L_{1}, L_{2}, L_{\infty}$ order tests of the trapezoid scheme and the third order semi-discrete scheme with different parameter $\tau$ value and the initial condition $u_{B}$ are given in Tables 4.1, 4.2. Table 4.1 shows that the trapezoid rule achieved second order accuracy for all the tested cases in $L_{1}, L_{2}, L_{\infty}$ sense. Table 4.2 shows that the semi-discrete scheme has the order of accuracy greater than 2.5 for all the cases, and exceeds 3 for some cases. This confirms the accuracy study given in sections 3.1.1 and 3.2 respectively. 
CHAPTER 4. COMPUTATIONAL RESULTS

\begin{tabular}{|c|c|c|c|c|c|c|c|}
\hline \multirow{5}{*}{$\begin{array}{l} \\
\\
u_{B}=0.9 \\
\tau=0.2\end{array}$} & \multirow{2}{*}{$\begin{array}{l}\mathrm{N} \\
60\end{array}$} & \multicolumn{2}{|c|}{$\left\|u_{\Delta x}-u_{\frac{\Delta x}{2}}\right\|_{1}$ order } & \multicolumn{2}{|c|}{$u_{\Delta x}-u_{\frac{\Delta x}{2}} \|_{2}$ order } & \multicolumn{2}{|c|}{$u_{\Delta x}-u_{\frac{\Delta x}{2}} \|_{\infty}$ order } \\
\hline & & $7.5416 \mathrm{e}-03$ & - & $2.5388 \mathrm{e}-03$ & - & $1.5960 \mathrm{e}-03$ & 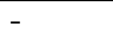 \\
\hline & 120 & $1.9684 \mathrm{e}-03$ & 1.9379 & $6.7288 \mathrm{e}-04$ & 1.9157 & $4.4066 \mathrm{e}-04$ & 1.8568 \\
\hline & 240 & $4.9891 \mathrm{e}-04$ & 1.9802 & $1.7645 \mathrm{e}-04$ & 1.9311 & $1.2529 \mathrm{e}-04$ & 1.8144 \\
\hline & 480 & $1.2589 \mathrm{e}-04$ & 1.9865 & $4.5366 \mathrm{e}-05$ & 1.9596 & $3.3205 \mathrm{e}-05$ & 1.9158 \\
\hline \multirow{4}{*}{$\begin{array}{l}u_{B}=0.9 \\
\tau=1\end{array}$} & 60 & $8.0141 \mathrm{e}-03$ & - & $2.6069 \mathrm{e}-03$ & - & $1.4989 \mathrm{e}-03$ & - \\
\hline & 120 & $2.1502 \mathrm{e}-03$ & 1.8981 & $7.0452 \mathrm{e}-04$ & 1.8876 & $4.2221 \mathrm{e}-04$ & 1.8279 \\
\hline & 240 & $5.5697 \mathrm{e}-04$ & 1.9488 & $1.8259 \mathrm{e}-04$ & 1.9480 & $1.1283 \mathrm{e}-04$ & 1.9038 \\
\hline & 480 & $1.4104 \mathrm{e}-04$ & 1.9815 & $4.6109 \mathrm{e}-05$ & 1.9855 & $2.8719 \mathrm{e}-05$ & 1.9740 \\
\hline \multirow{4}{*}{$\begin{array}{l}u_{B}=0.9 \\
\tau=5\end{array}$} & 60 & $1.3102 \mathrm{e}-02$ & - & $4.1784 \mathrm{e}-03$ & - & $2.2411 \mathrm{e}-03$ & - \\
\hline & 120 & $3.6201 \mathrm{e}-03$ & 1.8557 & $1.0994 \mathrm{e}-03$ & 1.9263 & $6.1060 \mathrm{e}-04$ & 1.8759 \\
\hline & 240 & $9.6737 \mathrm{e}-04$ & 1.9039 & $2.8089 \mathrm{e}-04$ & 1.9686 & $1.5667 \mathrm{e}-04$ & 1.9625 \\
\hline & 480 & $2.5825 \mathrm{e}-04$ & 1.9053 & $7.1250 \mathrm{e}-05$ & 1.9790 & $3.9286 \mathrm{e}-05$ & 1.9956 \\
\hline \multirow{4}{*}{$\begin{array}{l}u_{B}=\alpha \\
\tau=0.2\end{array}$} & 60 & $6.4427 \mathrm{e}-03$ & - & $2.1578 \mathrm{e}-03$ & - & $1.1682 \mathrm{e}-03$ & - \\
\hline & 120 & $1.6611 \mathrm{e}-03$ & 1.9555 & $5.7775 \mathrm{e}-04$ & 1.9011 & $3.6447 \mathrm{e}-04$ & 1.6804 \\
\hline & 240 & $4.3643 \mathrm{e}-04$ & 1.9283 & $1.5215 \mathrm{e}-04$ & 1.9250 & $1.0389 \mathrm{e}-04$ & 1.8107 \\
\hline & 480 & $1.1223 \mathrm{e}-04$ & 1.9593 & $3.9170 \mathrm{e}-05$ & 1.9577 & $2.7629 \mathrm{e}-05$ & 1.9109 \\
\hline \multirow{4}{*}{$\begin{array}{l}u_{B}=\alpha \\
\tau=1\end{array}$} & 60 & $7.5867 \mathrm{e}-03$ & - & $2.4101 \mathrm{e}-03$ & - & $1.3364 \mathrm{e}-03$ & 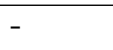 \\
\hline & 120 & $2.0069 \mathrm{e}-03$ & 1.9185 & $6.4998 \mathrm{e}-04$ & 1.8906 & $3.7650 \mathrm{e}-04$ & 1.8277 \\
\hline & 240 & $5.1832 \mathrm{e}-04$ & 1.9531 & $1.6801 \mathrm{e}-04$ & 1.9519 & $1.0062 \mathrm{e}-04$ & 1.9037 \\
\hline & 480 & $1.3136 \mathrm{e}-04$ & 1.9803 & $4.2497 \mathrm{e}-05$ & 1.9831 & $2.5599 \mathrm{e}-05$ & 1.9748 \\
\hline \multirow{4}{*}{$\begin{array}{l}u_{B}=\alpha \\
\tau=5\end{array}$} & 60 & $1.1959 \mathrm{e}-02$ & - & $3.8026 \mathrm{e}-03$ & - & $1.9938 \mathrm{e}-03$ & - \\
\hline & 120 & $3.2940 \mathrm{e}-03$ & 1.8602 & $9.9527 \mathrm{e}-04$ & 1.9338 & $5.4231 \mathrm{e}-04$ & 1.8783 \\
\hline & 240 & $8.7736 \mathrm{e}-04$ & 1.9086 & $2.5358 \mathrm{e}-04$ & 1.9727 & $1.3933 \mathrm{e}-04$ & 1.9606 \\
\hline & 480 & $2.3271 \mathrm{e}-04$ & 1.9146 & $6.4252 \mathrm{e}-05$ & 1.9806 & $3.4967 \mathrm{e}-05$ & 1.9944 \\
\hline \multirow{4}{*}{$\begin{array}{l}u_{B}=0.75 \\
\tau=0.2\end{array}$} & 60 & $5.7714 \mathrm{e}-03$ & - & $1.9358 \mathrm{e}-03$ & - & $1.0481 \mathrm{e}-03$ & - \\
\hline & 120 & $1.5035 \mathrm{e}-03$ & 1.9406 & $5.1617 \mathrm{e}-04$ & 1.9070 & $2.8061 \mathrm{e}-04$ & 1.9011 \\
\hline & 240 & $3.9299 \mathrm{e}-04$ & 1.9357 & $1.3616 \mathrm{e}-04$ & 1.9225 & 7.9134e-05 & 1.8262 \\
\hline & 480 & $1.0063 \mathrm{e}-04$ & 1.9655 & $3.5080 \mathrm{e}-05$ & 1.9566 & $2.1035 \mathrm{e}-05$ & 1.9115 \\
\hline \multirow{4}{*}{$\begin{array}{l}u_{B}=0.75 \\
\tau=1\end{array}$} & 60 & $7.1823 \mathrm{e}-03$ & - & $2.2843 \mathrm{e}-03$ & - & $1.2069 \mathrm{e}-03$ & - \\
\hline & 120 & $1.8963 \mathrm{e}-03$ & 1.9213 & $6.1315 \mathrm{e}-04$ & 1.8974 & $3.4013 \mathrm{e}-03$ & 1.8272 \\
\hline & 240 & $4.8284 \mathrm{e}-04$ & 1.9736 & $1.5796 \mathrm{e}-04$ & 1.9567 & $9.0912 \mathrm{e}-04$ & 1.9035 \\
\hline & 480 & $1.2093 \mathrm{e}-04$ & 1.9974 & $3.9783 \mathrm{e}-05$ & 1.9894 & $2.3121 \mathrm{e}-05$ & 1.9753 \\
\hline \multirow{4}{*}{$\begin{array}{l}u_{B}=0.75 \\
\tau=5\end{array}$} & 60 & $1.1042 \mathrm{e}-02$ & - & $3.5020 \mathrm{e}-03$ & - & $1.8299 \mathrm{e}-03$ & - \\
\hline & 120 & $3.0287 \mathrm{e}-03$ & 1.8662 & $9.1181 \mathrm{e}-04$ & 1.9414 & $4.8976 \mathrm{e}-04$ & 1.9016 \\
\hline & 240 & $8.0111 \mathrm{e}-04$ & 1.9186 & $2.3118 \mathrm{e}-04$ & 1.9797 & $1.2593 \mathrm{e}-04$ & 1.9595 \\
\hline & 480 & $2.1076 \mathrm{e}-04$ & 1.9264 & $5.8358 \mathrm{e}-05$ & 1.9860 & $3.1627 \mathrm{e}-05$ & 1.9934 \\
\hline
\end{tabular}

Table 4.1: The accuracy test for the trapezoid scheme for the MBL equation 4.1) with $\epsilon=1$ and $M=2$. 
CHAPTER 4. COMPUTATIONAL RESULTS

\begin{tabular}{|c|c|c|c|c|c|c|c|}
\hline & $N$ & $u_{\Delta x}-u$ & order & $u_{\Delta x}-u_{\frac{\Delta a}{2}}$ & order & $u_{\Delta x}-u_{2}$ & order \\
\hline \multirow{4}{*}{$\begin{array}{l}u_{B}=0.9 \\
\tau=0.2\end{array}$} & 120 & $2.6992 \mathrm{e}-03$ & - & $1.1300 \mathrm{e}-03$ & - & $7.2363 \mathrm{e}-04$ & - \\
\hline & 240 & $4.0403 \mathrm{e}-04$ & 2.7400 & $1.7079 \mathrm{e}-04$ & 2.7260 & $1.1283 \mathrm{e}-04$ & 2.6811 \\
\hline & 480 & $5.7504 \mathrm{e}-05$ & 2.8127 & $2.4624 \mathrm{e}-05$ & 2.7941 & $1.6242 \mathrm{e}-05$ & 2.7963 \\
\hline & 960 & $8.4934 \mathrm{e}-06$ & 2.7592 & $3.0892 \mathrm{e}-06$ & 2.9948 & $1.7607 \mathrm{e}-06$ & 3.2055 \\
\hline \multirow{4}{*}{$\begin{array}{l}u_{B}=0.9 \\
\tau=1\end{array}$} & 120 & $4.7731 \mathrm{e}-03$ & - & $2.0192 \mathrm{e}-03$ & - & $1.7267 \mathrm{e}-03$ & - \\
\hline & 240 & $8.7205 \mathrm{e}-04$ & 2.4524 & $3.6879 \mathrm{e}-04$ & 2.4529 & $3.0632 \mathrm{e}-04$ & 2.4949 \\
\hline & 480 & $1.2006 \mathrm{e}-04$ & 2.8606 & $5.0480 \mathrm{e}-05$ & 2.8690 & $4.1985 \mathrm{e}-05$ & 2.8671 \\
\hline & 960 & $1.5942 \mathrm{e}-05$ & 2.9129 & $6.6663 \mathrm{e}-06$ & 2.9208 & $5.1464 \mathrm{e}-06$ & 3.0282 \\
\hline \multirow{4}{*}{$\begin{array}{l}u_{B}=0.9 \\
\tau=5\end{array}$} & 120 & $3.7573 \mathrm{e}-03$ & - & $1.2122 \mathrm{e}-03$ & 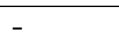 & 7.9211e-04 & - \\
\hline & 240 & 7.4624e-04 & 2.3320 & $2.4164 \mathrm{e}-04$ & 2.3267 & $1.5061 \mathrm{e}-04$ & 2.3949 \\
\hline & 480 & $1.1994 \mathrm{e}-04$ & 2.6373 & $3.8434 \mathrm{e}-05$ & 2.6524 & $2.5089 \mathrm{e}-05$ & 2.5857 \\
\hline & 960 & $1.5565 \mathrm{e}-05$ & 2.9460 & $4.9190 \mathrm{e}-06$ & 2.9660 & $3.1363 \mathrm{e}-06$ & 2.9999 \\
\hline \multirow{4}{*}{$\begin{array}{l}u_{B}=\alpha \\
\tau=0.2\end{array}$} & 120 & $2.1836 \mathrm{e}-03$ & - & $9.1039 \mathrm{e}-04$ & - & $5.7219 \mathrm{e}-04$ & - \\
\hline & 240 & $3.2729 \mathrm{e}-04$ & 2.7381 & $1.3760 \mathrm{e}-04$ & 2.7260 & $8.9550 \mathrm{e}-05$ & 2.6757 \\
\hline & 480 & $4.6856 \mathrm{e}-05$ & 2.8043 & $1.9909 \mathrm{e}-05$ & 2.7890 & $1.2935 \mathrm{e}-05$ & 2.7914 \\
\hline & 960 & $6.7382 \mathrm{e}-06$ & 2.7978 & $2.3182 \mathrm{e}-06$ & 3.1023 & $1.4109 \mathrm{e}-06$ & 3.1965 \\
\hline \multirow{4}{*}{$\begin{array}{l}u_{B}=\alpha \\
\tau=1\end{array}$} & 120 & $3.9014 \mathrm{e}-03$ & - & $1.6388 \mathrm{e}-03$ & - & $1.3873 \mathrm{e}-03$ & - \\
\hline & 240 & 7.0517e-04 & 2.4680 & $2.9669 \mathrm{e}-04$ & 2.4656 & $2.4272 \mathrm{e}-04$ & 2.5149 \\
\hline & 480 & $9.6528 \mathrm{e}-05$ & 2.8690 & $4.0354 \mathrm{e}-05$ & 2.8781 & $3.3125 \mathrm{e}-05$ & 2.8733 \\
\hline & 960 & $1.2890 \mathrm{e}-05$ & 2.9047 & $5.3648 \mathrm{e}-06$ & 2.9111 & $4.0754 \mathrm{e}-06$ & 3.0229 \\
\hline \multirow{4}{*}{$\begin{array}{l}u_{B}=\alpha \\
\tau=5\end{array}$} & 120 & $3.0797 \mathrm{e}-03$ & - & $9.9202 \mathrm{e}-04$ & - & $6.4456 \mathrm{e}-04$ & - \\
\hline & 240 & $6.1133 \mathrm{e}-04$ & 2.3328 & $1.9783 \mathrm{e}-04$ & 2.3261 & $1.2277 \mathrm{e}-04$ & 2.3924 \\
\hline & 480 & $9.7351 \mathrm{e}-05$ & 2.6507 & $3.1222 \mathrm{e}-05$ & 2.6637 & $2.0263 \mathrm{e}-05$ & 2.5990 \\
\hline & 960 & $1.2396 \mathrm{e}-05$ & 2.9733 & $3.9513 \mathrm{e}-06$ & 2.9822 & $2.4962 \mathrm{e}-06$ & 3.0210 \\
\hline \multirow{4}{*}{$\begin{array}{l}u_{B}=0.75 \\
\tau=0.2\end{array}$} & 120 & $1.8244 \mathrm{e}-03$ & - & $7.5548 \mathrm{e}-04$ & - & $4.6671 \mathrm{e}-04$ & - \\
\hline & 240 & $2.7262 \mathrm{e}-04$ & 2.7425 & $1.1419 \mathrm{e}-04$ & 2.7260 & $7.3299 \mathrm{e}-05$ & 2.6707 \\
\hline & 480 & $3.9198 \mathrm{e}-05$ & 2.7980 & $1.6562 \mathrm{e}-05$ & 2.7855 & $1.0681 \mathrm{e}-05$ & 2.7788 \\
\hline & 960 & $5.4739 \mathrm{e}-06$ & 2.8401 & $1.9677 \mathrm{e}-06$ & 3.0733 & $1.3232 \mathrm{e}-06$ & 3.0129 \\
\hline \multirow{4}{*}{$\begin{array}{l}u_{B}=0.75 \\
\tau=1\end{array}$} & 120 & $3.2727 \mathrm{e}-03$ & - & $1.3672 \mathrm{e}-03$ & - & $1.1477 \mathrm{e}-03$ & - \\
\hline & 240 & $5.8671 \mathrm{e}-04$ & 2.4798 & $2.4585 \mathrm{e}-04$ & 2.4754 & $1.9866 \mathrm{e}-04$ & 2.5304 \\
\hline & 480 & 7.9974e- 05 & 2.8750 & $3.3285 \mathrm{e}-05$ & 2.8848 & $2.7033 \mathrm{e}-05$ & 2.8775 \\
\hline & 960 & $1.0724 \mathrm{e}-05$ & 2.8987 & $4.4466 \mathrm{e}-06$ & 2.9041 & $3.3341 \mathrm{e}-06$ & 3.0193 \\
\hline \multirow{4}{*}{$\begin{array}{l}u_{B}=0.75 \\
\tau=5\end{array}$} & 120 & $2.5902 \mathrm{e}-03$ & - & $8.3335 \mathrm{e}-04$ & - & $5.3882 \mathrm{e}-04$ & - \\
\hline & 240 & $5.1342 \mathrm{e}-04$ & 2.3348 & $1.6611 \mathrm{e}-04$ & 2.3268 & $1.0271 \mathrm{e}-04$ & 2.3913 \\
\hline & 480 & $8.1062 \mathrm{e}-05$ & 2.6630 & $2.6032 \mathrm{e}-05$ & 2.6738 & $1.6813 \mathrm{e}-05$ & 2.6109 \\
\hline & 960 & $1.0173 \mathrm{e}-05$ & 2.9944 & $3.2662 \mathrm{e}-06$ & 2.9946 & $2.0473 \mathrm{e}-06$ & 3.0377 \\
\hline
\end{tabular}

Table 4.2: The accuracy test for the third order semi-discrete scheme for the MBL equation (4.1) with $\epsilon=1$ and $M=2$. 


\subsection{Traveling-Wave Solutions}

The numerical solutions to the MBL equation (4.1) we will calculate later in this chapter are the traveling-wave solutions. Van Duijn et al. [21] have proven the existence of the traveling wave solution in [21], for the completeness, we include it here. To look for traveling wave solution. Let

$$
\eta=\frac{x-s t}{\epsilon}
$$

where $s$ is given by the Rankine-Hugoniot condition (1.4) and $u_{l}$ and $u_{r}$ are the $u$ values to the left and to the right of the shock respectively.

Substituting (4.3) into 4.1), we obtain

$$
-s u^{\prime}+(f(u))^{\prime}=u^{\prime \prime}-s \tau u^{\prime \prime \prime} \quad \text { in } \mathcal{R}
$$

When we integrate this equation over $(\eta, \infty)$, we obtain the second order boundary value problem

$$
\begin{aligned}
& -s\left(u-u_{r}\right)+\left(f(u)-f\left(u_{r}\right)\right)=u^{\prime}-s \tau u^{\prime \prime} \quad \text { in } \mathcal{R} \\
& u(-\infty)=u_{l} \quad u(\infty)=u_{r}
\end{aligned}
$$

There're two cases to consider

- (I) $u_{r}=0, u_{l}>0$

- (II) $u_{r}>r_{l}>0$

Case I: $u_{r}=0$

Van Duijn et al. [21] has proven the following proposition. 
Proposition 4.2.1. Let $u$ be a solution of (4.4) such that $u_{r}=0$. Then $u_{l}<\beta$, where $\beta$ is the value of $u$ for which the equal area rule holds (see figure 4.1):

$$
\int_{0}^{\beta}\left\{f(u)-\frac{f(\beta)}{\beta} u\right\} d u=0
$$

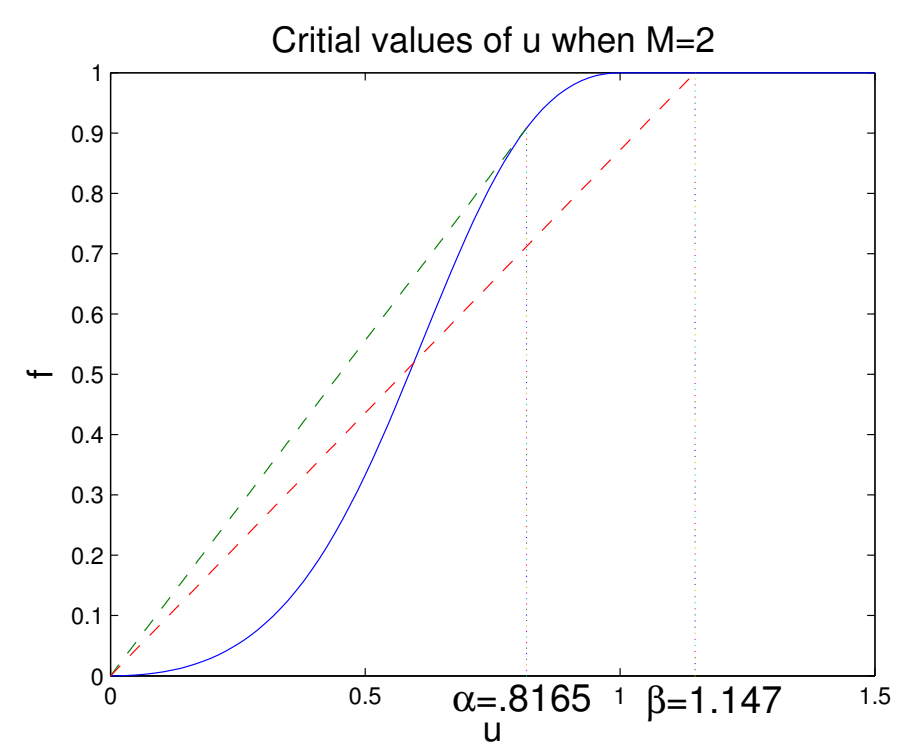

Figure 4.1: Critical values of $u$ when $M=2: \alpha \approx 0.816$ and $\beta \approx 1.147$.

Notice that if $u_{l} \in(\alpha, \beta)$, then

$$
s=s\left(u_{l}, 0\right)=\frac{f\left(u_{l}\right)}{u_{l}}>f^{\prime}\left(u_{l}\right) \geq f^{\prime}(0) \quad \text { for } u_{l}>\alpha
$$

and traveling waves, if they exist, lead to an admissibility condition for fast undercompressive waves. For convenience, it's denoted as $s\left(u_{l}, 0\right)=s\left(u_{l}\right)$ and $\alpha$ is given 
in (1.6). In the theorems below Van Duijn et al. showed that for each $\tau>0$, there exists a unique value of $u_{l} \geq \alpha$, denoted by $\bar{u}(\tau)$, for which there exists a solution of problem (4.4) such that $u_{r}=0$.

Theorem 4.2.2. Let $M>0$ be given. Then there exists a constant $\tau_{*}>0$ such that the following hold:

- (a) For each $0 \leq \tau \leq \tau_{*}$, problem (4.4) has a unique solution with $u_{l}=\alpha$ and $u_{r}=0$.

- (b) For each $\tau>\tau_{*}$, there exists a unique constant $\bar{u}_{l}(\tau) \in(\alpha, \beta)$ such that problem (4.4) has a unique solution with $u_{l}=\bar{u}_{l}(\tau)$ and $u_{r}=0$.

- (c) The function $\bar{u}:[0, \infty) \rightarrow[\alpha, \beta)$ defined by

$$
\bar{u}(\tau)=\left\{\begin{array}{lll}
\alpha & \text { for } & 0 \leq \tau \leq \tau_{*} \\
\bar{u}_{l}(\tau) & \text { for } \quad \tau>\tau_{*}
\end{array}\right.
$$

is continuous, strictly increasing for $\tau \geq \tau_{*}$, and $\bar{u}(\infty)=\beta$.

The solution in parts (a) and (b) are strictly decreasing.

$\bar{u}=\bar{u}(\tau)$ if usually referred as the plateau value of $u$, and the speed $s(\bar{u})$ of the shock $\{\bar{u}, 0\}$ is denoted by $\bar{s}$. In the case that $u_{l} \neq \bar{u}(\tau)$, another critical value of $u$ is introduced and is denoted by $\underline{u}$.

- For $\tau \in\left[0, \tau_{*}\right]$, we put $\underline{u}(\tau)=\alpha$

- For $\tau>\tau_{*}$, we define $\underline{u}(\tau)$ as the unique zero in the interval $(0, \bar{u}(\tau))$ of

$$
f(r)-\frac{f(\bar{u})}{\bar{u}} r=0 \quad 0<r<\bar{u}
$$


Notice that, if $\tau>\tau_{*}$, then

$$
0<\underline{u}(\tau)<\alpha<\bar{u}(\tau)<\beta \quad \text { for } \tau>\tau_{*}
$$

Theorem 4.2.3. Let $M>0$, and $\tau>0$ be given, and let $\underline{u}=\underline{u}(\tau)$ and $\bar{u}=\bar{u}(\tau)$.

- (a) For any $u_{l} \in(0, \underline{u})$, there exists a unique solution of (4.4) problem such that $u_{r}=0$. We have $s\left(u_{l}\right)<\bar{s}=s(\bar{u})$.

- (b) Let $\tau>\tau_{*}$. Then for any $u_{l} \in(\underline{u}, \bar{u})$, there exists no solution of (4.4) problem such that $u_{r}=0$.

The solution in part (a) may exhibit a damped oscillation as it tends to $u_{l}$.

Case II: $u_{r}>0$. The results of Case I raise the question as to how to deal with the solutions of problem (4.1) when $u_{B} \in(\underline{u}, \bar{u})$, and by Theorem 4.2.3, there is no traveling wave solution with $u_{r}=0$. In this situation, two traveling waves in succession are used: one from $u_{B}$ to the plateau value $\bar{u}$ and one from $\bar{u}$ down to $u=0$. The existence of the latter has been established in Theorem 4.2.2. In the next theorem, Van Duijn et al. dealt with the former, in which $u_{r}=\bar{u}$.

Theorem 4.2.4. Let $M>0$ and $\tau>\tau_{*}$ be given, and let $\underline{u}=\underline{u}(\tau)$ and $\bar{u}=\bar{u}(\tau)$.

- (a) For any $u_{l} \in(\underline{u}, \bar{u})$, there exists a unique traveling-wave solution of problem (4.4) such that $u_{r}=\bar{u}$. We have $s\left(u_{l}, \bar{u}\right)<\bar{s}$.

- (b) For any $u_{l} \in(0, \underline{u})$, there exists no solution of problem (4.4) such that $u_{r}=\bar{u}$. 
The solution in part (a) may exhibit a damped oscillation as it tends to $u_{l}$.

The proofs of Theorems 4.2.2 4.2.3 4.2 .4 are given in [21].

\subsection{Parameter Regimes}

For $M=2$, Duijn et al. [21] numerically provided a bifurcation diagram (we reproduced it in Figure 4.2 of MBL (4.1) equation as the dispersive parameter $\tau$ and the post-shock value $u_{B}$ of the initial condition vary. The solution of (4.1) has been proven to display qualitatively different profiles for parameter values $\left(\tau, u_{B}\right)$ falling in different regimes of the bifurcation diagram. In particular, for every fixed $\tau$ value,

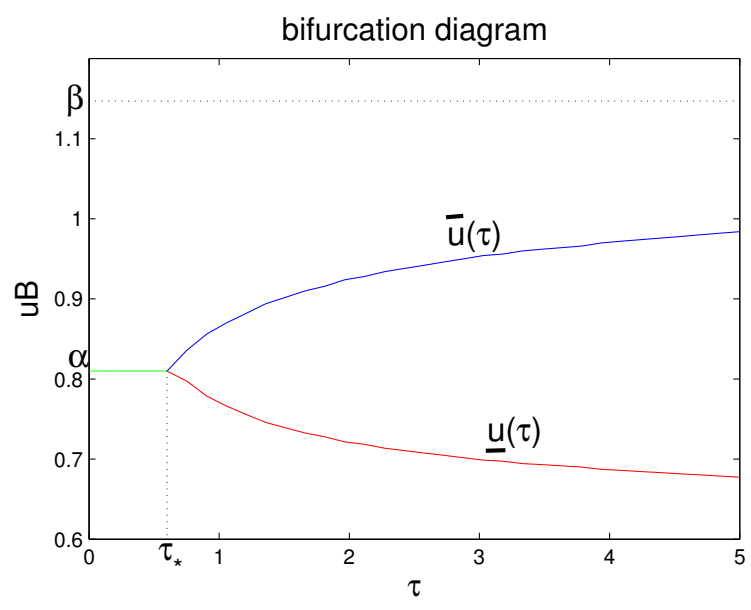

Figure 4.2: The bifurcation diagram of the MBL equation (4.1) with the bifurcation parameters $\left(\tau, u_{B}\right)$, where $M=2$. 
there are two critical $u_{B}$ values, namely, $\bar{u}$ and $\underline{u}$. From the bifurcation diagram (Figure 4.2), it is clear that, when $\tau<\tau_{*}, \bar{u}=\underline{u}=\alpha$. For a fixed $\tau$ value, the solution has three different profiles.

(a) If $u_{B} \in[\bar{u}, 1]$, the solution contains a plateau value $u_{B}$ for $0 \leq \frac{x}{t} \leq \frac{d f}{d u}\left(u_{B}\right)$, a rarefaction wave connection $u_{B}$ to $\bar{u}$ for $\frac{d f}{d u}\left(u_{B}\right) \leq \frac{x}{t} \leq \frac{d f}{d u}(\bar{u})$, another plateau value $\bar{u}$ for $\frac{d f}{d u}(\bar{u})<\frac{x}{t}<\frac{f(\bar{u})}{\bar{u}}$, and a shock from $\bar{u}$ down to 0 at $\frac{x}{t}=\frac{f(\bar{u})}{\bar{u}}$ (see Figure 4.3(a) .

(b) If $u_{B} \in(\underline{u}, \bar{u})$, the solution contains a plateau value $u_{B}$ for $0 \leq \frac{x}{t}<\frac{f(\bar{u})-f\left(u_{B}\right)}{\bar{u}-u_{B}}$, a shock from $u_{B}$ up to $\bar{u}$ at $\frac{x}{t}=\frac{f(\bar{u})-f\left(u_{B}\right)}{\bar{u}-u_{B}}$, another plateau value $\bar{u}$ for $\frac{f(\bar{u})-f\left(u_{B}\right)}{\bar{u}-u_{B}}<$ $\frac{x}{t}<\frac{f(\bar{u})}{\bar{u}}$, and a shock from $\bar{u}$ down to 0 at $\frac{x}{t}=\frac{f(\bar{u})}{\bar{u}}$ (see Figure 4.3(b)). The solution may exhibit a damped oscillation near $u=u_{B}$.

(c) If $u_{B} \in\left(0, \underline{u}\right.$ ], the solution consists a single shock connecting $u_{B}$ and 0 at $\frac{x}{t}=\frac{f\left(u_{B}\right)}{u_{B}}$ (see Figure $4.3(\mathrm{c})$. It may exhibit oscillatory behavior near $u=u_{B}$.

Notice that when $\tau>\tau_{*}$ and $\underline{u}<u_{B}<\bar{u}$, the solution profiles 4.3(b) displays non-monotonicity, which is consistent with the experimental observations ([8]).

\subsection{Examples}

In this section, we will use examples to study the solutions to MBL equation (4.1) using the numerical schemes proposed in chapter 3. We first notice that if we scale $t$ and $x$ as follows

$$
\tilde{t}=\frac{t}{\epsilon}, \quad \tilde{x}=\frac{x}{\epsilon}
$$


(a) $u_{B}>\bar{u}$

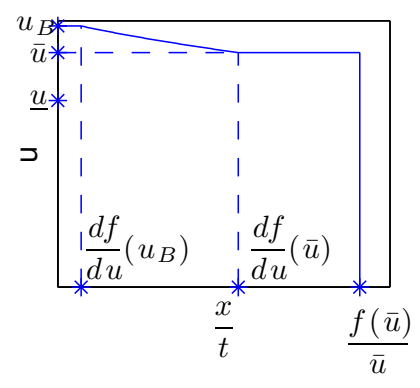

(b) $\underline{u}<u_{B}<\bar{u}$

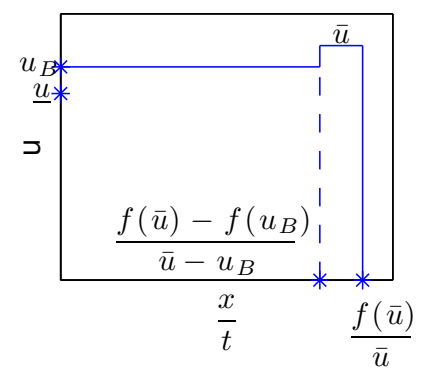

(c) $u_{B}<\underline{u}$

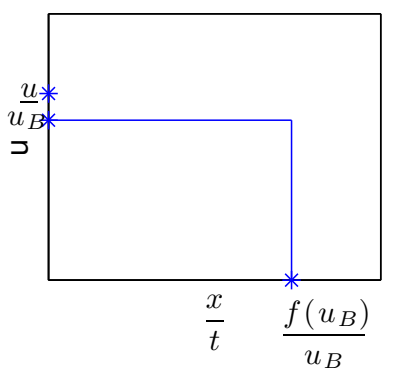

Figure 4.3: Given a fixed $\tau$, the three qualitatively different solution profiles due to different values of $u_{B}$. In particular, when $\tau>\tau_{*}$ and $\underline{u}<u_{B}<\bar{u}$, the solution profiles (Figure 4.3(b) displays non-monotonicity, which is consistent with the experimental observations ([8]). Figures 4.3(a), 4.3(b) and $4.3(\mathrm{c})$ are demonstrative figures.

then MBL 4.1 equation can be written in terms of $\tilde{t}$ and $\tilde{x}$ as follows

$$
u_{\tilde{t}}+(f(u))_{\tilde{x}}=u_{\tilde{x} \tilde{x}}+\tau u_{\tilde{x} \tilde{x} \tilde{t}}
$$

The scaled equation (4.7) shows that it is the magnitude of $\frac{t}{\epsilon}$ and $\frac{x}{\epsilon}$ that determine the asymptotic behavior, not $t, x$, neither $\epsilon$ alone ([21]). In addition, 4.7) also shows that the dispersive parameter $\tau$ denotes the relative importance of the dispersive term $u_{x x t}$. The bigger $\tau$ is, the more dispersive effect 4.1) equation has. This can be seen from the computational results to be shown later in this section.

In the numerical computation we show later, we will therefore test the accuracy and capability of central schemes for different parameter values $\left(\tau\right.$ and $\left.u_{B}\right)$ that fall into various regimes of the bifurcation diagram, and therefore display qualitatively different solution profiles. The numerical experiments were carried out for $M=2$, 
$\epsilon=0.001$ and $T=4000 \times \epsilon$, i.e. $\tilde{T}=4000$ to get the asymptotic solution profiles, and $\Delta x$ was chosen to be $\frac{\epsilon}{10}$ and $\lambda=\frac{\Delta t}{\Delta x}$ was chosen to be 0.1 . The scheme used in the computation is the second order Trapezoid scheme as shown in section 3.1.1. The Midpoint scheme delivers similar computational results, hence is omitted here. The solution profiles at $\frac{1}{4} T$ (blue), $\frac{2}{4} T$ (green), $\frac{3}{4} T$ (magenta) and $T$ (black) are chosen to demonstrate the time evolution of the solutions. The red dashed lines are used to denote the theoretical shock locations and plateau values for comparison purpose.

We start with $\tau>0$. Based on the bifurcation diagram (Figure 4.2), we choose three representative $u_{B}$ values, i.e. $u_{B}=0.9>\alpha, u_{B}=\alpha=\sqrt{\frac{M}{M+1}}=\sqrt{\frac{2}{3}}($ for $M=2$ ) and $u_{B}=0.75<\alpha$. For each fixed $u_{B}$, we choose three representative $\tau$ values, i.e. $\tau=0.2<\tau_{*} \approx 0.61, \tau=1>\tau_{*}$, (in this case, the three representative $u_{B}$ values $0.9, \alpha, 0.75$ are separated by $\bar{u}$ and $\underline{u}$ as follows $0.75<\underline{u}_{\tau=1}<\alpha<\bar{u}_{\tau=1}<0.9$ ), and $\tau=5$ with $u_{B}=0.75, \alpha, 0.9 \in\left[\underline{u}_{\tau=5}, \bar{u}_{\tau=5}\right]$. We first use these 9 pairs of $\left(\tau, u_{B}\right)$ values given in Table 4.3 to validate the solution profiles with the demonstrative solution profiles given in Figure 4.3 .

Example $1\left(\tau, u_{B}\right)=(0.2,0.9),\left(\tau, u_{B}\right)=(1,0.9),\left(\tau, u_{B}\right)=(5,0.9)$.

When $u_{B}=0.9>\alpha$ is fixed, we increase $\tau$ from 0.2 to 1 to 5 (Figure 4.4(a), 4.4(b) , 4.4(c), the dispersive effect starts to dominate the solution profile. When $\tau=0.2$ (Figure 4.4(a) , the solution profile is similar to the classical BL equation solution (see Figure 1.4(b)], with a rarefaction wave for $\frac{x}{t} \in\left[f^{\prime}(u=0.9), f^{\prime}(u=\alpha)=f^{\prime}(u=\right.$ $\left.\left.\bar{u}_{\tau=0.2}\right)\right]$ and a shock from $u=\alpha$ to $u=0$ at $\frac{x}{t}=f^{\prime}(\alpha)$. This corresponds to Figure 4.3(a) with $\frac{d f}{d u}\left(\bar{u}_{\tau=0.2}=\alpha\right)=\frac{f\left(\bar{u}_{\tau=0.2}\right)}{\bar{u}_{\tau=0.2}}=\frac{f(\alpha)}{\alpha}$. When $\tau=1$ (Figure 4.4(b), the rarefaction wave is between $\frac{x}{t} \in\left[f^{\prime}(u=0.9), f^{\prime}\left(u=\bar{u}_{\tau=1}\right)\right]$ and the solution remains 
CHAPTER 4. COMPUTATIONAL RESULTS

\begin{tabular}{|l|l|l|l|}
\hline$\left(\tau, u_{B}\right)$ & Example 4 & Example 5 & Example 6 \\
\hline Example 1 & $(0.2,0.9)$ & $(1,0.9)$ & $(5,0.9)$ \\
\hline Example 2 & $(0.2, \alpha)$ & $(1, \alpha)$ & $(5, \alpha)$ \\
\hline Example 3 & $(0.2,0.75)$ & $(1,0.75)$ & $(5,0.75)$ \\
\hline
\end{tabular}

Table 4.3: 9 pairs of $\left(\tau, u_{B}\right)$ values with either fixed $\tau$ value varying $u_{B}$ values or fixed $u_{B}$ value varying $\tau$ values used in Examples $1-6$. Notice that $\alpha=\sqrt{\frac{M}{M+1}}=\sqrt{\frac{2}{3}}$ for $M=2$.

at the plateau value $u=\bar{u}_{\tau=1}$ for $\frac{x}{t} \in\left[f^{\prime}\left(u=\bar{u}_{\tau=1}\right), \frac{f\left(\bar{u}_{\tau=1}\right)}{\bar{u}_{\tau=1}}\right]$ and the shock occurs at $\frac{x}{t}=\frac{f\left(\bar{u}_{\tau=1}\right)}{\bar{u}_{\tau=1}}$. This corresponds to Figure $4.3(\mathrm{a})$ with $u_{B}=0.9>\bar{u}_{\tau=1} \approx 0.86$. When $\tau=5$ (Figure 4.4(c)) , the solution displays the first shock from $u=0.9$ to $u=\bar{u}_{\tau=5}$ at $\frac{x}{t}=\frac{f\left(\bar{u}_{\tau=5}\right)-f\left(u_{B}\right)}{\bar{u}_{\tau=5}-u_{B}}$, and then remains at the plateau value $u=\bar{u}_{\tau=5}$ for $\frac{x}{t} \in\left[\frac{f\left(\bar{u}_{\tau=5}\right)-f\left(u_{B}\right)}{\bar{u}_{\tau=5}-u_{B}}, \frac{f\left(\bar{u}_{\tau=5}\right)}{\bar{u}_{\tau=5}}\right]$ and the second shocks occurs at $\frac{x}{t}=\frac{f\left(\bar{u}_{\tau=5}\right)}{\bar{u}_{\tau=5}}$. This corresponds to Figure 4.3(b) with $\underline{u}_{\tau=5} \approx 0.68<u_{B}=0.9<\bar{u}_{\tau=5} \approx 0.98$. Notice that as $\tau$ increases, the rarefaction region shrinks and the plateau region enlarges.

Example $2\left(\tau, u_{B}\right)=(0.2, \alpha),\left(\tau, u_{B}\right)=(1, \alpha),\left(\tau, u_{B}\right)=(5, \alpha)$.

When $u_{B}=\alpha$ is fixed, we increase $\tau$ from 0.2 to 1 to 5 (Figure 4.4(d), 4.4(e), $4.4(\mathrm{f})$, the dispersive effect starts to dominate the solution profile. When $\tau=0.2$, the solution displays one single shock at $\frac{x}{t}=\frac{f(\alpha)}{\alpha}$. For both $\tau=1$ and $\tau=5$,

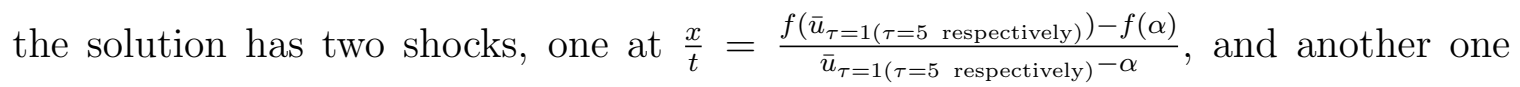
at $\frac{x}{t}=\frac{f\left(\bar{u}_{\tau=1(\tau=5} \text { respectively) }\right)}{\bar{u}_{\tau=1(\tau=5} \text { respectively) }}$. For both $\tau=1$ and $\tau=5$ (Figures 4.4(e) 4.4(f) , 
(a) $\left(\tau, u_{B}\right)=(0.2,0.9)$

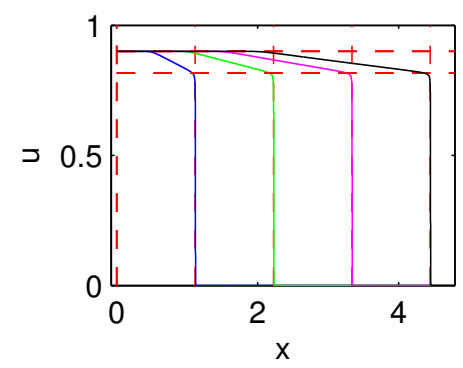

(d) $\left(\tau, u_{B}\right)=(0.2, \alpha)$

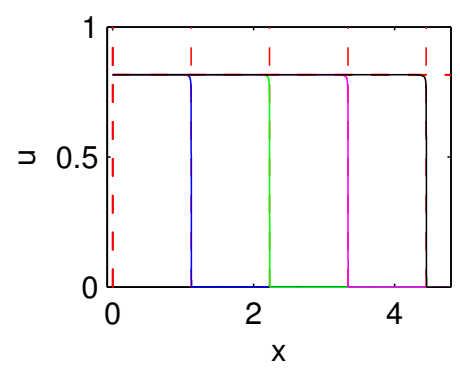

(g) $\left(\tau, u_{B}\right)=(0.2,0.75)$

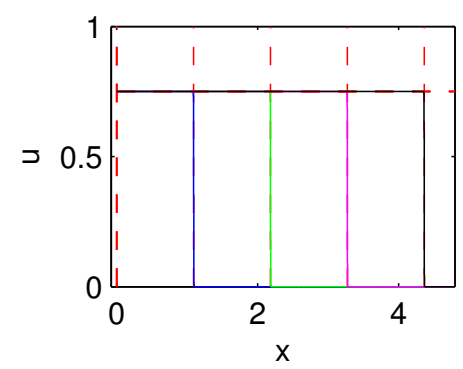

(b) $\left(\tau, u_{B}\right)=(1,0.9)$

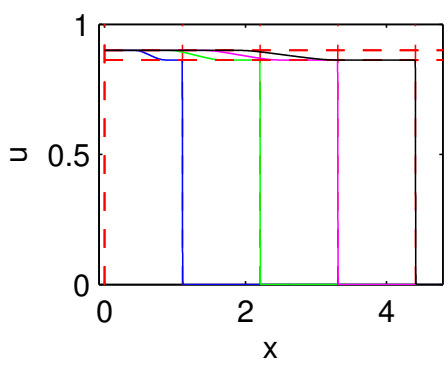

(e) $\left(\tau, u_{B}\right)=(1, \alpha)$

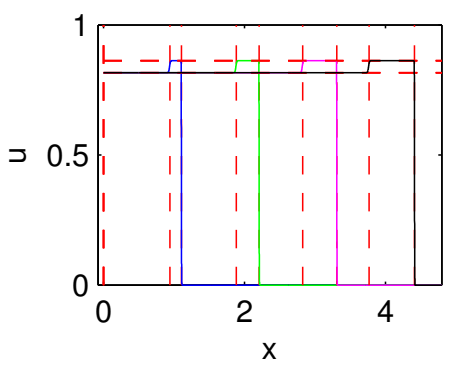

(h) $\left(\tau, u_{B}\right)=(1,0.75)$

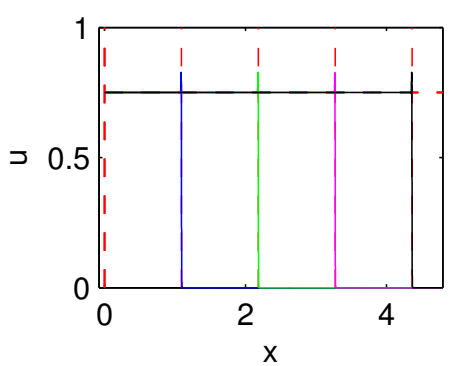

(c) $\left(\tau, u_{B}\right)=(5,0.9)$

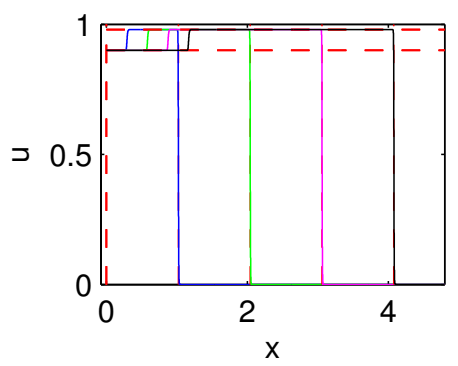

(f) $\left(\tau, u_{B}\right)=(5, \alpha)$

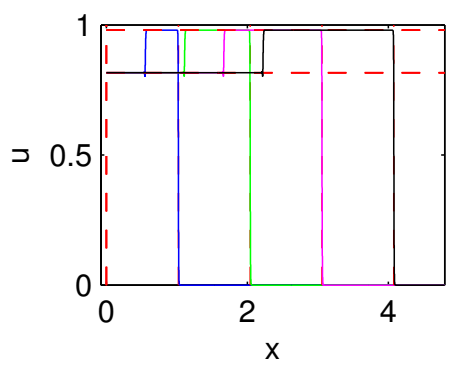

(i) $\left(\tau, u_{B}\right)=(5,0.75)$

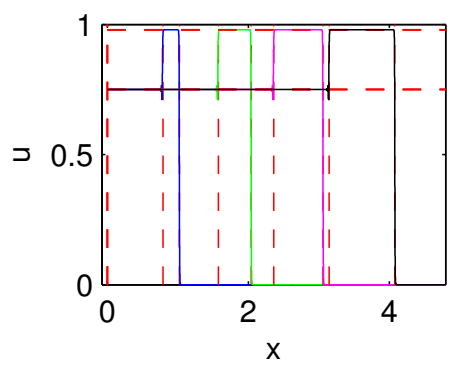

Figure 4.4: Numerical solutions to MBL equation with parameter settings fall in different regimes of the bifurcation diagram (Figure 4.2). The color coding is for different time: $\frac{1}{4} T$ (blue), $\frac{2}{4} T$ (green), $\frac{3}{4} T$ (magenta) and $T$ (black). The results are discussed in examples $1-6$. In figures 4.4(d) $-4.4(\mathrm{f})$, $\alpha=\sqrt{\frac{M}{M+1}}=\sqrt{\frac{2}{3}}$ for $M=2$. 
the solutions correspond to Figure 4.3(b), which are consistent to the experimental observations. Notice that as $\tau$ increases from 1 to 5 , i.e., the dispersive effect increases, the inter-shock interval length increases at every fixed time (compare Figure 4.4(e) with Figure 4.4(f) $)$. In addition, for fix $\tau=1$ ( $\tau=5$ respectively), as time progresses, the inter-shock interval length increases in the linear fashion (see Figure 4.4(e) (Figure $4.4(\mathrm{f})$ respectively) ).

Example $3\left(\tau, u_{B}\right)=(0.2,0.75),\left(\tau, u_{B}\right)=(1,0.75),\left(\tau, u_{B}\right)=(5,0.75)$.

When $u_{B}=0.75<=\alpha$ is fixed, we increase $\tau$ from 0.2 to 1 to 5 (Figure $4.4(\mathrm{~g})$, 4.4(h), 4.4(i) , the dispersive effects starts to dominate the solution profile in the similar fashion as $u_{B}=0.9$ and $u_{B}=\alpha$. Notice that when $\tau=1$, since $u_{B}=0.75$ is very close to $\underline{u}_{\tau=1}$, the solution displays oscillation at $\frac{x}{t}=\frac{f\left(u_{B}\right)}{u_{B}}$ (Figure 4.4(h). If we increase $\tau$ further to $\tau=5$, the dispersive effect is strong enough to create a plateau value at $\bar{u} \approx 0.98$ (see Figure 4.4(i)) .

Example $4\left(\tau, u_{B}\right)=(0.2,0.9),\left(\tau, u_{B}\right)=(0.2, \alpha),\left(\tau, u_{B}\right)=(0.2,0.75)$.

Now, we fix $\tau=0.2$, decrease $u_{B}$ from 0.9 to $\alpha$, to 0.75 (Figures4.4(a) $4.4(\mathrm{~d}) 4.4(\mathrm{~g})$ ) . If $u_{B}>\alpha$ the solution consists a rarefaction wave connecting $u_{B}$ down to $\alpha$, then a shock from $\alpha$ to 0 , otherwise, the solution consists a single shock from $u_{B}$ down to 0 . In all cases, since $\tau=0.2<\tau_{*}$, regardless of the $u_{B}$ value, the solution will not display non-monotone behavior, due to the lack of dispersive effect.

Example $5\left(\tau, u_{B}\right)=(1,0.9),\left(\tau, u_{B}\right)=(1, \alpha),\left(\tau, u_{B}\right)=(1,0.75)$.

Now, we fix $\tau=1$, decrease $u_{B}$ from 0.9 to $\alpha$, to 0.75 (Figures 4.4(b) 4.4(e) 4.4(h)) . If $u_{B}=0.9>\bar{u}_{\tau=1}$, the solution consists a rarefaction wave connecting $u_{B}$ and $\bar{u}$, and a shock connecting $\bar{u}$ down to 0 (Figure 4.4(b)). Even if $\underline{u}<u_{B}<\bar{u}$, because 
$\tau=1>\tau_{*}$, the solution still has a chance to increase to the plateau value $\bar{u}$ as seen in Figure 4.4(e), But, if $u_{B}$ is too small, for example, $u_{B}=0.75<\underline{u}$, the solution does not increase to $\bar{u}$ any more, instead, it consists a single shock connecting $u_{B}$ down to 0 (Figure 4.4(h)).

Example $6\left(\tau, u_{B}\right)=(5,0.9),\left(\tau, u_{B}\right)=(5, \alpha),\left(\tau, u_{B}\right)=(5,0.75)$.

Now, we fix $\tau=5$, decrease $u_{B}$ from 0.9 to $\alpha$, to 0.75 (Figures4.4(c)|4.4(f)|4.4(i)]. For all three $u_{B}$, they are between $\underline{u}_{\tau=5}$ and $\bar{u}_{\tau=5}$, hence all increase to the plateau value $\bar{u}_{\tau=5} \approx 0.98$ before dropping to 0 . Notice that as $u_{B}$ decreases, the inter-shock interval length decreases at every fixed time (compare Figures 4.4(c), 4.4(f) and 4.4(i)). This shows that when the dispersive effect is strong $\left(\tau>\tau_{*}\right)$, the bigger $u_{B}$ is, the bigger region the solution stays at the plateau value.

Example $7\left(\tau, u_{B}\right)=(0,0.9),\left(\tau, u_{B}\right)=(0, \alpha),\left(\tau, u_{B}\right)=(0,0.75)$.

We now show the solution profiles for the extreme $\tau$ value, i.e. $\tau=0$ in Figures $4.5(\mathrm{a})$ $\left(u_{B}=0.9\right), 4.5(\mathrm{~b})\left(u_{B}=\alpha\right)$ and $4.5(\mathrm{c})\left(u_{B}=0.75\right)$. Notice that these are cases of classical BL equation with small diffusion $\epsilon u_{x x}$. We compare Figures 4.5(a), 4.5(b) and $4.5(\mathrm{c})$ with the solution of the classical BL equation given in Figures 1.4(a) and 1.4(b), it is clear that they show qualitatively same solution profiles. The difference is that due to the diffusion term in the MBL equation, the solutions in Figure 4.5 do not have sharp edges right at the shock, instead, the solutions smear out a little. If we compare Figures 4.5(a), 4.5(b) and 4.5(c) with Figures Figures 4.4(a), 4.4(d) and $4.4(\mathrm{~g})$, there is no visible difference. This shows that once $\tau<\tau_{*}$, solution profile will stay the same for a fixed $u_{B}$ value.

Example $8\left(\tau, u_{B}\right)=(5,0.99),\left(\tau, u_{B}\right)=(5,0.98),\left(\tau, u_{B}\right)=(5,0.97)$. 
(a) $\left(\tau, u_{B}\right)=(0,0.9)$

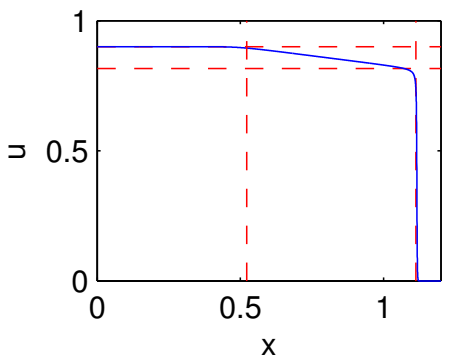

(b) $\left(\tau, u_{B}\right)=(0, \alpha)$

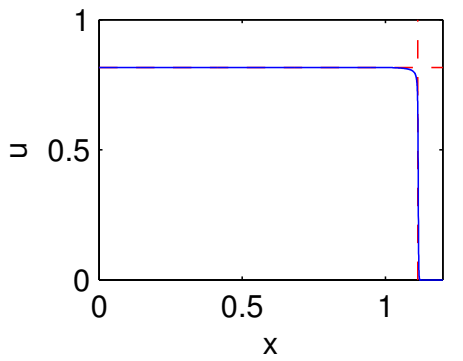

(c) $\left(\tau, u_{B}\right)=(0,0.75)$

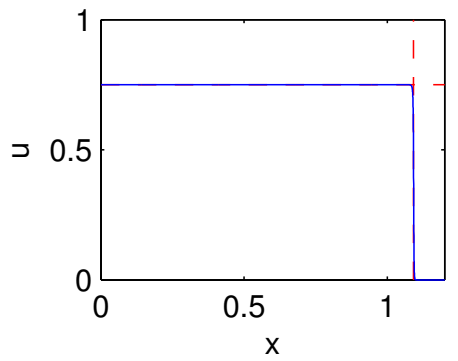

Figure 4.5: The numerical solutions of the MBL equation at $\mathrm{T}=1$ with $\tau=0$ and different $u_{B}$ values. The results are discussed in example 7 .

We also study the solution profiles for $u_{B}$ close to $\bar{u}$. For example, when $\tau=5$, $\bar{u} \approx 0.98$, we hence choose $u_{B}=0.99, u_{B}=0.98, u_{B}=0.97$ and solutions are shown in Figure 4.6(a), 4.6(b), 4.6(c), If $u_{B}=0.99>\bar{u}_{\tau=5} \approx 0.98$, the solution drops to the plateau value $\bar{u}$, then drops to 0 (see Figure $4.6(\mathrm{a})$. If $u_{B}=0.98 \approx \bar{u}_{\tau=5}$, the solution remains at plateau value $\bar{u}_{\tau=5}$ and then drop to 0 (see Figure 4.6(b)]. If $u_{B}=0.97<\bar{u}_{\tau=5}$, the solution increases to the plateau value $\bar{u}_{\tau=5} \approx 0.98$, then drops to 0 . In all cases, the transition from $u_{B}$ to $\bar{u}_{\tau=5} \approx 0.98$ takes very small space. In the majority space, the solution keeps to be the plateau value $\bar{u}_{\tau=5} \approx 0.98$.

Example $9\left(\tau, u_{B}\right)=(5,0.7),\left(\tau, u_{B}\right)=(5,0.69),\left(\tau, u_{B}\right)=(5,0.68),\left(\tau, u_{B}\right)=$ $(5,0.67),\left(\tau, u_{B}\right)=(5,0.66)$.

In addition, we study the solution profiles for $u_{B}$ close to $\underline{u}$. For example, when $\tau=5$, $\underline{u} \approx 0.68$, we hence choose $u_{B}=0.7, u_{B}=0.69, u_{B}=0.68, u_{B}=0.67, u_{B}=0.66$ and solutions are shown in Figures 4.7(a), 4.7(b), 4.7(c), 4.7(d), 4.7(e), As $u_{B}$ decreases crossing $\underline{u}_{\tau=5} \approx 0.68$, the solution gradually stops increasing to the plateau value 
(a) $\left(\tau, u_{B}\right)=(5,0.99)$

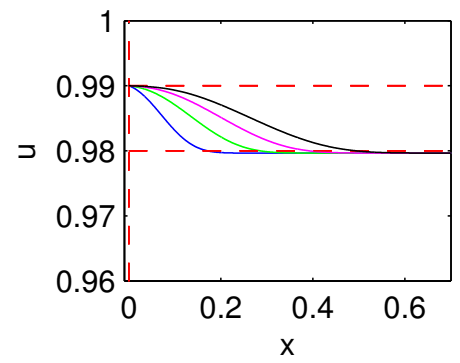

(b) $\left(\tau, u_{B}\right)=(5,0.98)$

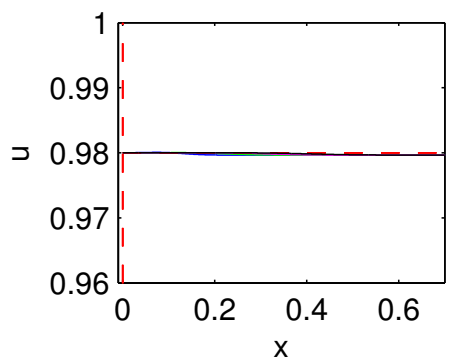

(c) $\left(\tau, u_{B}\right)=(5,0.97)$

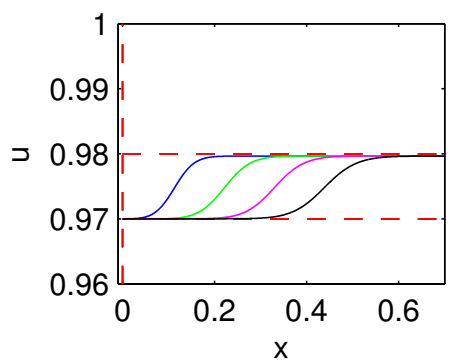

Figure 4.6: Numerical solutions to MBL equation with $u_{B}$ close to $\bar{u}_{\tau=5} \approx 0.98$. The color coding is for different time: $\frac{1}{4} T$ (blue), $\frac{2}{4} T$ (green), $\frac{3}{4} T$ (magenta) and $T$ (black). The results are discussed in example 8.

$\bar{u}_{\tau=5}$, and the inter-shock interval length decreases (compare Figures 4.7(a), 4.7(b) and 4.7(c) . The oscillation in Figures 4.7(d) and 4.7(e) are due to the fact that $u_{B}$ values are too close to $\underline{u}_{\tau=5}$. This confirms that even with big dispersive effect (say $\tau=5$ ), if $u_{B}$ is too small (e.g. $u_{B}<\underline{u}$ ), the solution will not exhibit non-monotone behavior.

Example $10\left(\tau, u_{B}\right)=(0.2,0.6),\left(\tau, u_{B}\right)=(1,0.6),\left(\tau, u_{B}\right)=(5,0.6)$.

We fix $u_{B}$ to be small, and in this example, we take it to be $u_{B}=0.6$. We vary the $\tau$ value, from $\tau=0.2<\tau_{*}$ to $\tau=1$ barely larger than $\tau_{*}$ to $\tau=5>\tau_{*}$. The numerical solutions are given in Figure 4.8(a), 4.8(b), 4.8(c), As $\tau$ increases, the post-shock value remains the same, but there will be oscillation generated as $\tau$ becomes larger than $\tau_{*}$. Figures $4.8(\mathrm{~d})$, $4.8(\mathrm{e})$ and $4.8(\mathrm{f})$ show that as $\tau$ increases, the oscillation amplitude increases and oscillates more rounds. Notice that $\tau$ is the dispersive parameter, and this means that even for small $u_{B}$ value, different dispersive 
(a) $\left(\tau, u_{B}\right)=(5,0.7)$

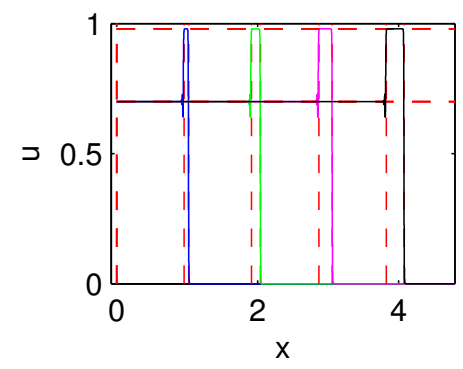

(d) $\left(\tau, u_{B}\right)=(5,0.67)$

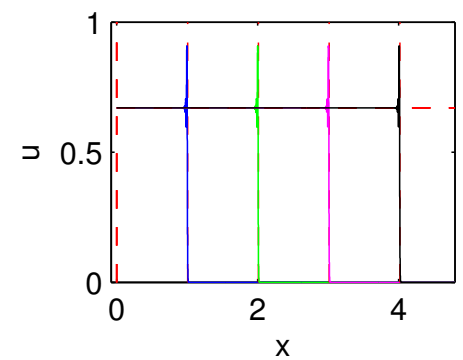

(b) $\left(\tau, u_{B}\right)=(5,0.69)$

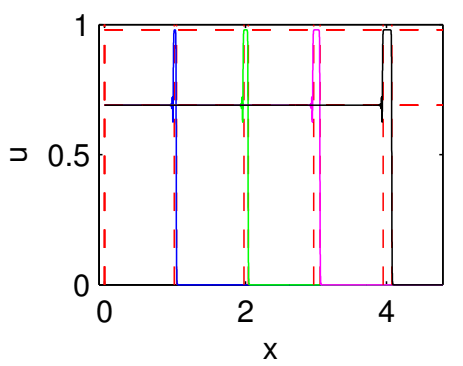

(e) $\left(\tau, u_{B}\right)=(5,0.66)$

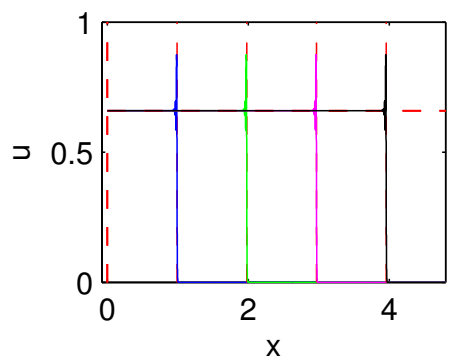

(c) $\left(\tau, u_{B}\right)=(5,0.68)$

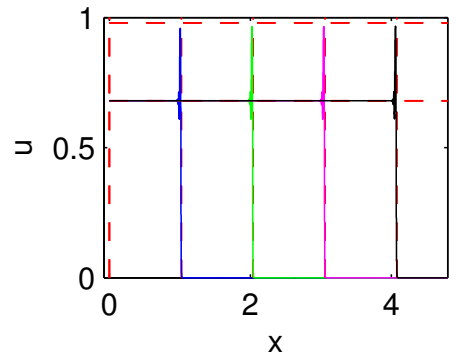

Figure 4.7: Numerical solutions to MBL equation with $u_{B}$ close to $\underline{u}_{\tau=5} \approx 0.68$. The color coding is for different time: $\frac{1}{4} T$ (blue), $\frac{2}{4} T$ (green), $\frac{3}{4} T$ (magenta) and $T$ (black). The results are discussed in example 9. 
parameter values still give different dispersive effects, although none can bring the solution to the plateau value $\bar{u}$. Comparing Figures 4.8(d), 4.8(e) and 4.8(f) with Figures 4.8(g), 4.8(h) and 4.8(i), it is clear that the oscillation amplitude remains steady with respect to time.

Example $11 \epsilon=0.001, \epsilon=0.002, \epsilon=0.003, \epsilon=0.004, \epsilon=0.005$.

In this example, we will compare the solution profiles for different $\epsilon$ values. Fixing $T=0.5, \Delta x=0.0001, \lambda=\frac{\Delta t}{\Delta x}=0.1$, we show the numerical results in Figure 4.9 for $\epsilon=0.001$ (blue), $\epsilon=0.002$ (yellow), $\epsilon=0.003$ (magenta), $\epsilon=0.004$ (green), and $\epsilon=0.005$ (black). For the purpose of cross reference, we choose the same nine sets of parameter settings as in examples 1-6. To assist the observation, the figures in Figure 4.9 are zoomed into the regions where different $\epsilon$ values introduce different solution profiles. The numerical solutions clearly show that as $\epsilon$ increases, the numerical solution is smeared out, and the jump location becomes less accurate. Notice that $\tau$ is responsible for the competition between the diffusion and dispersion, which in turn determines the plateau values. Hence varying $\epsilon$ value doesn't affect the plateau location. 
(a) $\left(\tau, u_{B}\right)=(0.2,0.6)$

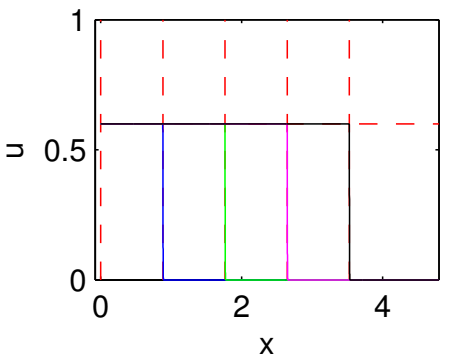

(d) Fig 4.8(a) zoomed in at $\frac{1}{4} T$

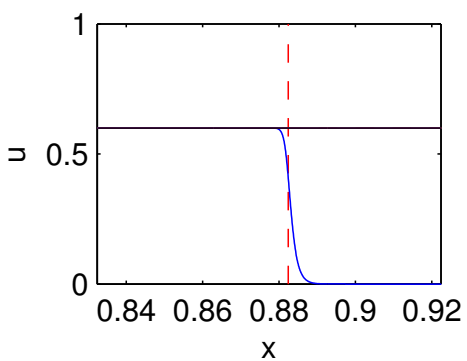

(g) Fig 4.8(a) zoomed in at $T$

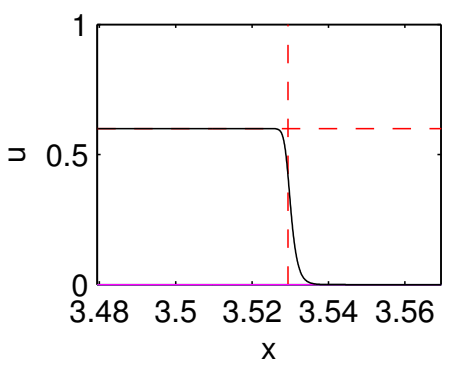

(b) $\left(\tau, u_{B}\right)=(1,0.6)$

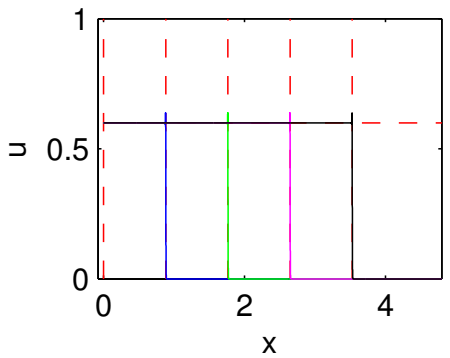

(e) Fig 4.8(b) zoomed in at $\frac{1}{4} T$

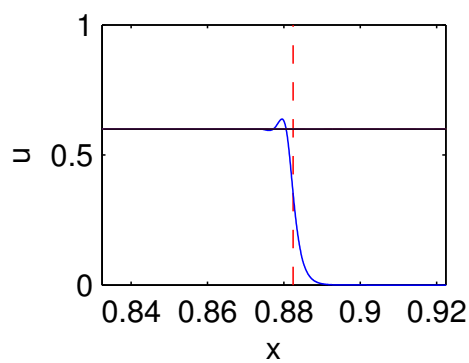

(h) Fig 4.8(b) zoomed in at $T$

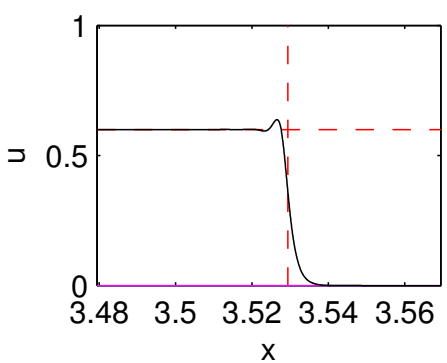

(c) $\left(\tau, u_{B}\right)=(5,0.6)$

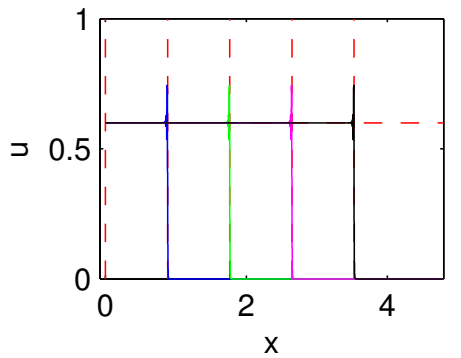

(f) Fig $4.8(\mathrm{c})$ zoomed in at $\frac{1}{4} T$

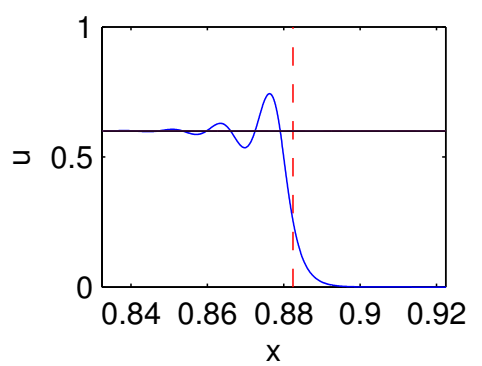

(i) Fig $4.8(\mathrm{c})$ zoomed in at $T$

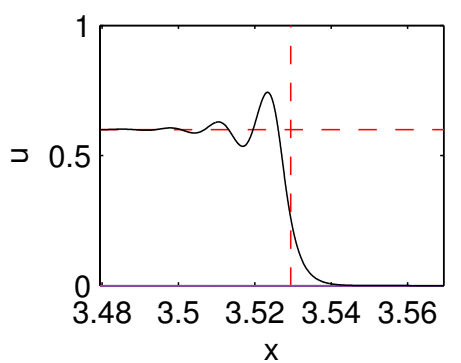

Figure 4.8: Numerical solutions to MBL equation with small constant $u_{B}=0.6$ and different $\tau$ values. The figures on the second and third rows are the magnified versions of the first row at $t=\frac{1}{4} T$ and $t=T$ respectively. The color coding is for different time: $\frac{1}{4} T$ (blue), $\frac{2}{4} T$ (green), $\frac{3}{4} T$ (magenta) and $T$ (black). The results are discussed in examples 10 . 
(a) $\left(\tau, u_{B}\right)=(0.2,0.9)$

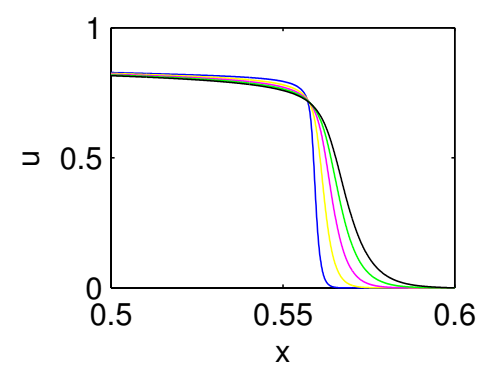

(d) $\left(\tau, u_{B}\right)=(0.2, \alpha)$

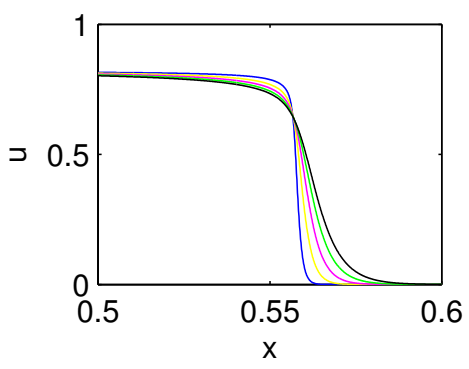

(g) $\left(\tau, u_{B}\right)=(0.2,0.75)$

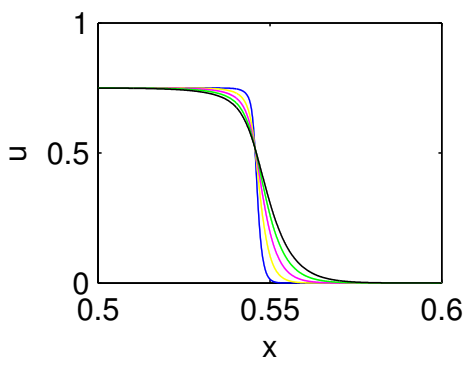

(b) $\left(\tau, u_{B}\right)=(1,0.9)$

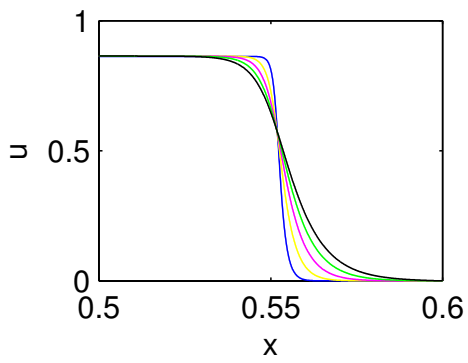

(e) $\left(\tau, u_{B}\right)=(1, \alpha)$

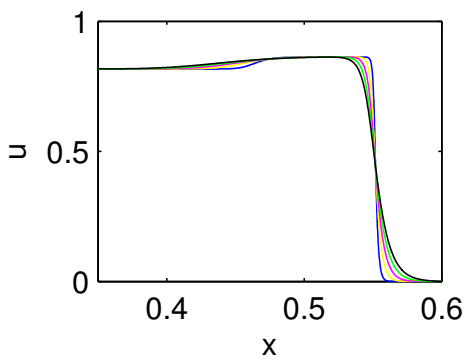

(h) $\left(\tau, u_{B}\right)=(1,0.75)$

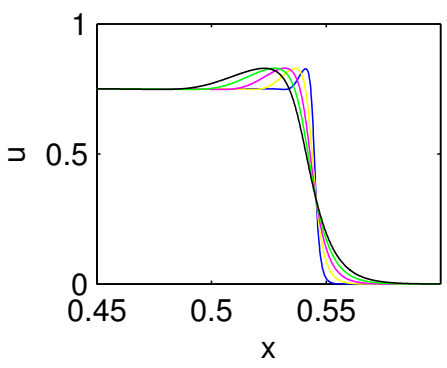

(c) $\left(\tau, u_{B}\right)=(5,0.9)$

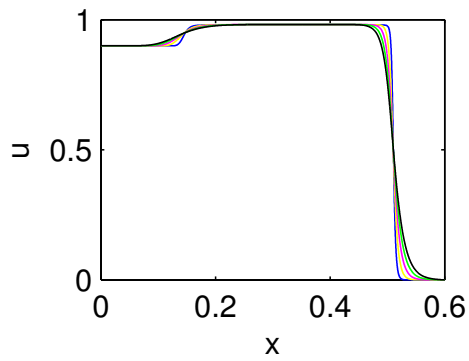

(f) $\left(\tau, u_{B}\right)=(5, \alpha)$

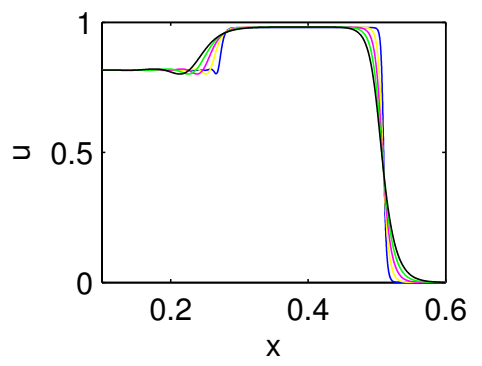

(i) $\left(\tau, u_{B}\right)=(5,0.75)$

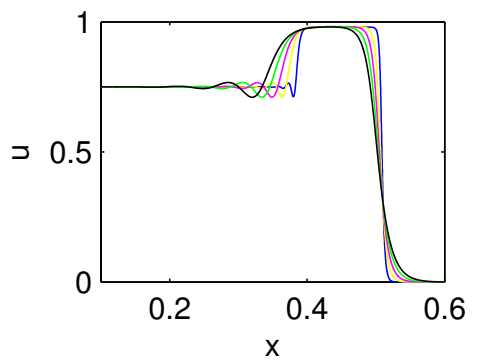

Figure 4.9: The numerical solutions of MBL equation at $T=0.5$ with $\epsilon=0.001$ (blue), $\epsilon=0.002$ (yellow), $\epsilon=0.003$ (magenta), $\epsilon=0.004$ (green), and $\epsilon=0.005$ (black). The view windows are zoomed into the regions where different $\epsilon$ values impose different solution profiles. The results are discussed in example 11. 


\section{CHAPTER 5 \\ TWO-DIMENSIONAL MODIFIED BUCKLEY-LEVERETT EQUATION}

\subsection{Derivation of the 2D MBL Equation}

To derive the two-dimensional extension of MBL equation, we consider the flow where imbibition takes places under influence of gravity [7]. We assume that the porous medium is homogeneous and isotropic, hence the mass balance gives

$$
\phi \partial_{t}\left(\rho_{i} S_{i}\right)+\nabla \cdot\left(\rho_{i} \overrightarrow{q_{i}}\right)=0, \quad i=o, w
$$

Both phases - oil and water - are considered to be incompressible, so

$$
\phi \partial_{t}\left(S_{i}\right)+\nabla \cdot\left(\overrightarrow{q_{i}}\right)=0, \quad i=o, w
$$

Notice that we again assume completion of saturation, i.e.

$$
S_{o}+S_{w}=1
$$

This in turn gives that

$$
\nabla \cdot\left(\overrightarrow{q_{o}}+\overrightarrow{q_{w}}\right)=\nabla \cdot \vec{q}=0
$$


Throughout this thesis, we assume $\vec{q}=$ const. By Darcy's law, the momentum balance equation is

$$
\overrightarrow{q_{i}}=\lambda_{i}\left(\nabla P_{i}+\rho_{i} g \overrightarrow{g_{z}}\right),
$$

where

$$
\begin{aligned}
\lambda_{i} & =-k \frac{k_{r_{i}}\left(S_{i}\right)}{\mu_{i}} \\
\rho_{i} & =\text { density of phase } i, \quad i=o, w, \\
g & =\text { gravitational constant, } \\
\overrightarrow{e_{z}} & =\text { unit vector in positive } z \text {-direction. }
\end{aligned}
$$

As in (1.13), we use the capillary pressure formulation proposed by Hassanizadeh and Gray [9] 10

$$
P_{c}=P_{o}-P_{w}=p_{c}\left(S_{w}\right)-\phi \tau \frac{\partial S_{w}}{\partial t}
$$

where $p_{c}\left(S_{w}\right)$ is the static capillary pressure and $\tau$ is a positive constant, and $\frac{\partial S_{w}}{\partial t}$ is the dynamic effects. For the ease of notation, we let

$$
\text { r.h.s. }=p_{c}\left(S_{w}\right)-\phi \tau \frac{\partial S_{w}}{\partial t}
$$

then

$$
\nabla P_{o}-\nabla P_{w}=\nabla \text { r.h.s. }
$$

From (5.3), we have that

$$
\nabla P_{o}-\nabla P_{w}=\frac{\overrightarrow{q_{o}}}{\lambda_{o}}-\rho_{o} g \overrightarrow{e_{z}}-\frac{\overrightarrow{q_{w}}}{\lambda_{w}}+\rho_{w} g \overrightarrow{e_{z}} .
$$


Combine (5.10) and (5.11), we can get

$$
\frac{\overrightarrow{q_{o}}}{\lambda_{o}}-\frac{\overrightarrow{q_{w}}}{\lambda_{w}}=\nabla \text { r.h.s. }-\left(\rho_{w}-\rho_{o}\right) g \overrightarrow{e_{z}} .
$$

Because of (5.2), we have that

$$
\begin{aligned}
\frac{\overrightarrow{q_{o}}}{\lambda_{o}}-\frac{\overrightarrow{q_{w}}}{\lambda_{w}} & =\frac{\vec{q}-\overrightarrow{q_{w}}}{\lambda_{o}}-\frac{\overrightarrow{q_{w}}}{\lambda_{w}} \\
& =\frac{\vec{q}}{\lambda_{o}}-\frac{\lambda_{o}+\lambda_{w}}{\lambda_{o} \lambda_{w}} \overrightarrow{q_{w}} .
\end{aligned}
$$

Therefore, (5.12) and (5.13) give that

$$
\frac{\vec{q}}{\lambda_{o}}-\frac{\lambda_{o}+\lambda_{w}}{\lambda_{o} \lambda_{w}} \overrightarrow{q_{w}}=\nabla \text { r.h.s. }-\left(\rho_{w}-\rho_{o}\right) g \overrightarrow{e_{z}} .
$$

Simplify (5.14), we have

$$
\overrightarrow{q_{w}}=\frac{\lambda_{w}}{\lambda_{o}+\lambda_{w}} \vec{q}-\frac{\lambda_{o} \lambda_{w}}{\lambda_{o}+\lambda_{w}}\left(\nabla \text { r.h.s }-\left(\rho_{w}-\rho_{o}\right) g \overrightarrow{e_{z}}\right) .
$$

Plug (5.15) into the governing equation for $S_{w}$ (5.1), and assume water is incompressible, we have that

$$
\phi \partial_{t}\left(S_{w}\right)+\nabla \cdot\left[\frac{\lambda_{w}}{\lambda_{o}+\lambda_{w}} q-\frac{\lambda_{o} \lambda_{w}}{\lambda_{o}+\lambda_{w}}\left(\nabla \text { r.h.s }-\left(\rho_{w}-\rho_{o}\right) g e_{z}\right)\right]=0,
$$

and hence

$$
\partial_{t}\left(S_{w}\right)+\nabla \cdot\left[\frac{\lambda_{w}}{\lambda_{o}+\lambda_{w}} \frac{\vec{q}}{\phi}+\frac{\lambda_{w}}{\lambda_{o}+\lambda_{w}} \frac{\lambda_{o}}{\phi}\left(\rho_{w}-\rho_{o}\right) g \overrightarrow{e_{z}}\right]=\nabla \cdot\left[\frac{\lambda_{o} \lambda_{w}}{\phi\left(\lambda_{o}+\lambda_{w}\right)} \nabla \text { r.h.s. }\right]
$$

Since (5.4) gives that

$$
\begin{aligned}
\frac{\lambda_{w}}{\lambda_{o}+\lambda_{w}} & =\frac{k_{r_{w}}\left(S_{w}\right)}{k_{r_{w}}\left(S_{w}\right)+\frac{\mu_{w}}{\mu_{o}} k_{r_{o}}\left(S_{o}\right)} \\
\lambda_{o} & =-k \frac{k_{r_{o}}\left(s_{o}\right)}{\mu_{o}}
\end{aligned}
$$


if we take

$$
k_{r_{o}}\left(S_{o}\right)=S_{o}^{2}, \quad k_{r_{w}}\left(S_{w}\right)=S_{w}^{2}
$$

and denote

$$
u=S_{w}
$$

then (5.16) gets us

$$
\partial_{t} u+\nabla \cdot\left[f(u) \frac{\vec{q}}{\phi}-f(u)(1-u)^{2} \frac{k\left(\rho_{w}-\rho_{o}\right) g}{\mu_{o} \phi} \overrightarrow{e_{z}}\right]=-\nabla \cdot\left[H(u) \nabla\left(J(u)-\tau \partial_{t} u\right)\right]
$$

where

$$
\begin{aligned}
f(u) & =\frac{u^{2}}{u^{2}+M(1-u)^{2}}, & M & =\frac{\mu_{w}}{\mu_{o}} \\
H(u) & =\frac{f(u) k(1-u)^{2}}{\mu_{0}}, & J(u) & =\frac{p_{c}(u)}{\phi} .
\end{aligned}
$$

This is the general form the two-dimensional extension of the Buckley-Leverett equation. In [13], the two-dimensional classical BL equation was used as an exam

$$
u_{t}+(f(u))_{x}+(g(u))_{z}=0
$$

where

$$
\begin{aligned}
& f(u)=\frac{u^{2}}{u^{2}+M(1-u)^{2}}, \quad M=\frac{\mu_{w}}{\mu_{o}}, \\
& g(u)=f(u)\left(1-5(1-u)^{2}\right) .
\end{aligned}
$$


Two-dimensional MBL equation (5.17) can be taken to a particular form, which is consistent to 5.18, To do that, we rescale

$$
\begin{aligned}
x \frac{\phi}{q_{1}} & \rightarrow x \\
z \frac{\phi}{q_{2}} & \rightarrow z
\end{aligned}
$$

and take

$$
\begin{aligned}
\frac{k\left(\rho_{w}-\rho_{o}\right) g}{\mu_{o} q_{2}} & =5 \\
\phi & =\mathcal{O}(\epsilon),
\end{aligned}
$$

then (5.17) becomes

$$
\partial_{t} u+\nabla \cdot\left[f(u) \overrightarrow{1}-5 f(u)(1-u)^{2} \vec{e}_{z}\right]=\epsilon \Delta u+\epsilon^{2} \tau \Delta u_{t},
$$

i.e.

$$
u_{t}+(f(u))_{x}+(g(u))_{z}=\epsilon \Delta u+\epsilon^{2} \tau \Delta u_{t}
$$

where $f(u)$ and $g(u)$ are defined as in 5.20. This equation is the two-dimensional modified Buckley-Leverett (MBL) equation extension of the 2D classical BuckleyLeverett equation [13]. 


\section{CHAPTER 6 CONCLUSION}

We started this thesis with one-dimensional Buckley-Leverett (BL) equation. After derivation of the modified BL equation (MBL), we proved that the solution to the infinite domain problem can be approximated by that of the bounded domain problem for the MBL equation. This provides a theoretical justification for using a finite domain to calculate the numerical solution of the MBL equation (1.19).

We also extended the classical central scheme originally designed for the hyperbolic systems to solve the MBL equation, which is of pseudo-parabolic type. The numerical solutions for qualitatively different parameter values $\tau$ and initial conditions $u_{B}$ show that the jump locations are consistent with the theoretical calculation and the plateau heights are consistent with the numerically obtained values given in [21]. In particular, when $\tau>\tau_{*}$, for $u_{B} \in(\underline{u}, \bar{u})$, the numerical solutions give non-monotone water saturation profiles, which is consistent with the experimental observations. In addition, the order tests show that the proposed second and third order central schemes achieved the desired accuracies.

We also provided the derivation of $2 \mathrm{D}$ modified $\mathrm{BL}$ equation, which is consistent to the 2D classical BL equation. Many more aspects of the modified BL equation can be studied. Here, we list three different topics. First, one can develop the bifurcation 
diagram for the 2D modified BL equation and study the connection between the $1 \mathrm{D}$ and 2D cases. Second, one can extend the proposed second and third order schemes to numerically solve the $2 \mathrm{D}$ modified BL equation 5.21, and validate the bifurcation diagram for the 2D MBL equation. Last but not least, the asymptotical stability of the traveling wave solutions of the MBL equation can be discussed. The further study of the MBL equation is not limited to the above mentioned. 


\section{Appendix A}

\section{PROOF OF THE LEMMAS}

\section{A.1 Proof to lemma 2.3.1}

Proof. Let $g(u)=\frac{f(u)}{u}=\frac{u}{u^{2}+M(1-u)^{2}}$, then

$$
g^{\prime}(u)=\frac{M-(1+M) u^{2}}{\left(u^{2}+M(1-u)^{2}\right)^{2}}\left\{\begin{array}{lll}
>0 & \text { if } & 0<u<\sqrt{\frac{M}{M+1}} \\
=0 & \text { if } & u=\sqrt{\frac{M}{M+1}} \\
<0 & \text { if } & u>\sqrt{\frac{M}{M+1}}
\end{array}\right.
$$

and hence $g(u)$ achieves its maximum at $u=\sqrt{\frac{M}{M+1}}$. Therefore, $\frac{f(u)}{u}=g(u) \leq D$, where $D=\frac{f(\alpha)}{\alpha}$ and $\alpha=\sqrt{\frac{M}{M+1}}$, and in turn, we have that $f(u) \leq D u$ for all $0 \leq u \leq 1$ 


\section{A.2 Proof to lemma 2.3.2}

Proof to lemma 2.3.2 (i).

$$
\begin{aligned}
& \int_{0}^{+\infty}\left|e^{-\frac{x+\xi}{\epsilon \sqrt{\tau}}}-e^{-\frac{|x-\xi|}{\epsilon \sqrt{\tau}}}\right| e^{\frac{\lambda x-\lambda \xi}{\epsilon \sqrt{\tau}}} d \xi \\
& =\int_{0}^{+\infty}\left(e^{-\frac{|x-\xi|}{\epsilon \sqrt{\tau}}}-e^{-\frac{x+\xi}{\epsilon \sqrt{\tau}}}\right) e^{\frac{\lambda x-\lambda \xi}{\epsilon \sqrt{\tau}}} d \xi \\
& =\int_{0}^{x}\left(e^{-\frac{x-\xi}{\epsilon \sqrt{\tau}}}-e^{-\frac{x+\xi}{\epsilon \sqrt{\tau}}}\right) e^{\frac{\lambda x-\lambda \xi}{\epsilon \sqrt{\tau}}} d \xi+\int_{x}^{+\infty}\left(e^{\frac{x-\xi}{\epsilon \sqrt{\tau}}}-e^{-\frac{x+\xi}{\epsilon \sqrt{\tau}}}\right) e^{\frac{\lambda x-\lambda \xi}{\epsilon \sqrt{\tau}}} d \xi \\
& =\frac{\left[e^{\frac{(\lambda-1)(x-\xi)}{\epsilon \sqrt{\tau}}}\right]_{\xi=0}^{\xi=x}}{-\frac{\lambda-1}{\epsilon \sqrt{\tau}}}-\frac{\left[e^{\frac{(\lambda-1) x-(\lambda+1) \xi}{\epsilon \sqrt{\tau}}}\right]_{\xi=0}^{\xi=x}}{-\frac{\lambda+1}{\epsilon \sqrt{\tau}}}+\frac{\left[e^{\frac{(\lambda+1)(x-\xi)}{\epsilon \sqrt{\tau}}}\right]_{\xi=x}^{\xi=+\infty}}{-\frac{\lambda+1}{\epsilon \sqrt{\tau}}}-\frac{\left[e^{\frac{(\lambda-1) x-(\lambda+1) \xi}{\epsilon \sqrt{\tau}}}\right]_{\xi=x}^{\xi=+\infty}}{-\frac{\lambda+1}{\epsilon \sqrt{\tau}}} \\
& =\frac{1-e^{\frac{(\lambda-1) x}{\epsilon \sqrt{\tau}}}}{-\frac{\lambda-1}{\epsilon \sqrt{\tau}}}-\frac{e^{\frac{-2 x}{\epsilon \sqrt{\tau}}}-e^{\frac{(\lambda-1) x}{\epsilon \sqrt{\tau}}}}{-\frac{\lambda+1}{\epsilon \sqrt{\tau}}}+\frac{0-1}{-\frac{\lambda+1}{\epsilon \sqrt{\tau}}}-\frac{0-e^{\frac{-2 x}{\epsilon \sqrt{\tau}}}}{-\frac{\lambda+1}{\epsilon \sqrt{\tau}}} \\
& =\frac{1-e^{\frac{(\lambda-1) x}{\epsilon \sqrt{\tau}}}}{-\frac{\lambda-1}{\epsilon \sqrt{\tau}}}-\frac{e^{-\frac{2 x}{\epsilon \sqrt{\tau}}}-e^{\frac{(\lambda-1) x}{\epsilon \sqrt{\tau}}}+1-e^{-\frac{2 x}{\epsilon \sqrt{\tau}}}}{-\frac{\lambda+1}{\epsilon \sqrt{\tau}}} \\
& =\epsilon \sqrt{\tau} \frac{-(\lambda+1)+(\lambda+1) e^{\frac{(\lambda-1) x}{\epsilon \sqrt{\tau}}}-(\lambda-1) e^{\frac{(\lambda-1) x}{\epsilon \sqrt{\tau}}}+(\lambda-1)}{\lambda^{2}-1} \\
& =\epsilon \sqrt{\tau} \frac{-2+2 e^{\frac{(\lambda-1) x}{\epsilon \sqrt{\tau}}}}{\lambda^{2}-1} \\
& \leq \frac{2 \epsilon \sqrt{\tau}}{1-\lambda^{2}}
\end{aligned}
$$


Proof to lemma 2.3.2 (iii).

$$
\begin{aligned}
& \int_{0}^{+\infty}\left|e^{-\frac{x+\xi}{\epsilon \sqrt{\tau}}}-e^{-\frac{|x-\xi|}{\epsilon \sqrt{\tau}}}\right| e^{\frac{\lambda x-\xi}{\epsilon \sqrt{\tau}}} d \xi \\
= & \int_{0}^{+\infty}\left(e^{-\frac{|x-\xi|}{\epsilon \sqrt{\tau}}}-e^{-\frac{x+\xi}{\epsilon \sqrt{\tau}}}\right) e^{\frac{\lambda x-\xi}{\epsilon \sqrt{\tau}}} d \xi \\
= & \int_{0}^{x}\left(e^{-\frac{x-\xi}{\epsilon \sqrt{\tau}}}-e^{-\frac{x+\xi}{\sqrt{\tau}}}\right) e^{\frac{\lambda x-\xi}{\epsilon \sqrt{\tau}}} d \xi+\int_{x}^{+\infty}\left(e^{\frac{x-\xi}{\epsilon \sqrt{\tau}}}-e^{-\frac{x+\xi}{\sqrt{\tau}}}\right) e^{\frac{\lambda x-\xi}{\epsilon \sqrt{\tau}}} d \xi \\
= & x e^{\frac{(\lambda-1) x}{\epsilon \sqrt{\tau}}}-\frac{\left[e^{\frac{(\lambda-1) x-2 \xi}{\epsilon \sqrt{\tau}}}\right]_{\xi=0}^{\xi=x}}{\frac{-2}{\epsilon \sqrt{\tau}}}+\frac{\left[e^{\frac{(\lambda+1) x-2 \xi}{\epsilon \sqrt{\tau}}}\right]_{\xi=x}^{\xi=+\infty}}{\frac{-2}{\epsilon \sqrt{\tau}}}-\frac{\left[e^{\frac{(\lambda-1) x-2 \xi}{\epsilon \sqrt{\tau}}}\right]_{\xi=x}^{\xi=+\infty}}{\frac{-2}{\epsilon \sqrt{\tau}}} \\
= & x e^{\frac{(\lambda-1) x}{\epsilon \sqrt{\tau}}} .
\end{aligned}
$$

Let

$$
F(x)=x e^{\frac{(\lambda-1) x}{\epsilon \sqrt{\tau}}},
$$

then

$$
\begin{aligned}
F^{\prime}(x) & =e^{\frac{(\lambda-1) x}{\epsilon \sqrt{\tau}}}+x e^{\frac{(\lambda-1) x}{\epsilon \sqrt{\tau}}} \frac{\lambda-1}{\epsilon \sqrt{\tau}} \\
& =e^{\frac{(\lambda-1) x}{\epsilon \sqrt{\tau}}}\left(1+x \frac{\lambda-1}{\epsilon \sqrt{\tau}}\right) .
\end{aligned}
$$

Assume $0<\lambda<1$, then

$$
F^{\prime}(x)\left\{\begin{array}{lll}
>0 & \text { if } & 0<x<\frac{\epsilon \sqrt{\tau}}{1-\lambda} \\
=0 & \text { if } & x=\frac{\epsilon \sqrt{\tau}}{1-\lambda} \\
<0 & \text { if } & x>\frac{\epsilon \sqrt{\tau}}{1-\lambda}
\end{array}\right.
$$

Hence,

$$
\begin{aligned}
F_{\max } & =F\left(\frac{\epsilon \sqrt{\tau}}{1-\lambda}\right) \\
& =\frac{\epsilon \sqrt{\tau}}{e(1-\lambda)}
\end{aligned}
$$


Therefore,

$$
\int_{0}^{+\infty}\left|e^{-\frac{x+\xi}{\epsilon \sqrt{\tau}}}-e^{-\frac{|x-\xi|}{\epsilon \sqrt{\tau}}}\right| e^{\frac{\lambda x-\xi}{\epsilon \sqrt{\tau}}} d \xi \leq \frac{\epsilon \sqrt{\tau}}{e(1-\lambda)}
$$

Proof to lemma 2.3.2 (iii). Based on the assumption on $u_{0}$ in 2.30

$$
\begin{aligned}
& \int_{0}^{+\infty}\left|e^{-\frac{x+\xi}{\epsilon \sqrt{\tau}}}-e^{-\frac{|x-\xi|}{\epsilon \sqrt{\tau}}}\right| e^{\frac{\lambda x}{\epsilon \sqrt{\tau}}}\left|u_{0}(\xi)\right| d \xi \\
\leq & \int_{0}^{+\infty} e^{-\frac{|x-\xi|}{\epsilon \sqrt{\tau}}} e^{\frac{\lambda x}{\epsilon \sqrt{\tau}}}\left|u_{0}(\xi)\right| d \xi \\
\leq & C_{u} e^{\frac{\lambda x}{\sqrt{\tau}}} \int_{0}^{L_{0}} e^{-\frac{|x-\xi|}{\epsilon \sqrt{\tau}}} d \xi \\
= & C_{u} y_{1}(x)
\end{aligned}
$$

Calculating $y_{1}(x)$ with the assumption that $\lambda \in(0,1)$, we get

- case (a): $x \in\left[0, L_{0}\right]$

$$
\begin{aligned}
y_{1}(x) & =\int_{0}^{L_{0}} e^{-\frac{|x-\xi|}{\epsilon \sqrt{\tau}}} e^{\frac{\lambda x}{\epsilon \sqrt{\tau}}} d \xi \\
& \leq \int_{0}^{x} e^{-\frac{x-\xi}{\epsilon \sqrt{\tau}}} e^{\frac{\lambda x}{\epsilon \sqrt{\tau}}} d \xi+\int_{x}^{L_{0}} e^{-\frac{\xi-x}{\epsilon \sqrt{\tau}}} e^{\frac{\lambda x}{\epsilon \sqrt{\tau}}} d \xi \\
& =\epsilon \sqrt{\tau}\left[e^{\frac{(\lambda-1) x+\xi}{\epsilon \sqrt{\tau}}}\right]_{\xi=0}^{\xi=x}+(-\epsilon \sqrt{\tau})\left[e^{\frac{(\lambda+1) x-\xi}{\epsilon \sqrt{\tau}}}\right]_{\xi=x}^{\xi=L_{0}} \\
& =\epsilon \sqrt{\tau}\left[e^{\frac{\lambda x}{\epsilon \sqrt{\tau}}}-e^{\frac{(\lambda-1) x}{\epsilon \sqrt{\tau}}}-e^{\frac{(\lambda+1) x-L_{0}}{\epsilon \sqrt{\tau}}}+e^{\frac{\lambda x}{\epsilon \sqrt{\tau}}}\right] \\
& \leq 2 \epsilon \sqrt{\tau} e^{\frac{\lambda x}{\epsilon \sqrt{\tau}}} \\
& \leq 2 \epsilon \sqrt{\tau} e^{\frac{\lambda L_{0}}{\epsilon \sqrt{\tau}}}
\end{aligned}
$$


- case (b): $x \in\left[L_{0},+\infty\right]$

$$
\begin{aligned}
y_{1}(x) & =\int_{0}^{L_{0}} e^{-\frac{x-\xi}{\epsilon \sqrt{\tau}}} e^{\frac{\lambda x}{\epsilon \sqrt{\tau}}} d \xi \\
& =\epsilon \sqrt{\tau}\left[e^{\frac{(\lambda-1) x+\xi}{\epsilon \sqrt{\tau}}}\right]_{\xi=0}^{\xi=L_{0}} \\
& =\epsilon \sqrt{\tau}\left(e^{\frac{(\lambda-1) x+L_{0}}{\epsilon \sqrt{\tau}}}-e^{\frac{(\lambda-1) x}{\epsilon \sqrt{\tau}}}\right) \\
& \leq \epsilon \sqrt{\tau} e^{\frac{(\lambda-1) x+L_{0}}{\epsilon \sqrt{\tau}}} \\
& =\epsilon \sqrt{\tau} e^{\frac{\lambda L_{0}}{\epsilon \sqrt{\tau}}} \\
& \leq 2 \epsilon \sqrt{\tau} e^{\frac{\lambda L_{0}}{\epsilon \sqrt{\tau}}}
\end{aligned}
$$

Therefore, we get the desired inequality

$$
\int_{0}^{+\infty}\left|e^{-\frac{x+\xi}{\epsilon \sqrt{\tau}}}-e^{-\frac{|x-\xi|}{\epsilon \sqrt{\tau}}}\right| e^{\frac{\lambda x}{\epsilon \sqrt{\tau}}}\left|u_{0}(\xi)\right| d \xi \leq 2 C_{u} \epsilon \sqrt{\tau} e^{\frac{\lambda L_{0}}{\epsilon \sqrt{\tau}}}
$$

$\square$ 


\section{A.3 Proof to lemma 2.3.3}

Proof to lemma 2.3.3 (i).

$$
\begin{aligned}
& \int_{0}^{+\infty}\left|e^{-\frac{x+\xi}{\epsilon \sqrt{\tau}}}+\operatorname{sgn}(x-\xi) e^{-\frac{|x-\xi|}{\epsilon \sqrt{\tau}}}\right| e^{\frac{\lambda x-\lambda \xi}{\epsilon \sqrt{\tau}}} d \xi \\
& =\int_{0}^{x}\left|e^{-\frac{x+\xi}{\epsilon \sqrt{\tau}}}+e^{-\frac{x-\xi}{\epsilon \sqrt{\tau}}}\right| e^{\frac{\lambda x-\lambda \xi}{\epsilon \sqrt{\tau}}} d \xi+\int_{x}^{+\infty}\left|e^{-\frac{x+\xi}{\epsilon \sqrt{\tau}}}-e^{-\frac{\xi-x}{\epsilon \sqrt{\tau}}}\right| e^{\frac{\lambda x-\lambda \xi}{\epsilon \sqrt{\tau}}} d \xi \\
& =\int_{0}^{x} e^{\frac{(\lambda-1) x-(\lambda+1) \xi}{\epsilon \sqrt{\tau}}}+e^{\frac{(\lambda-1) x+(1-\lambda) \xi}{\epsilon \sqrt{\tau}}} d \xi+\int_{x}^{+\infty} e^{\frac{(\lambda+1)(x-\xi)}{\epsilon \sqrt{\tau}}}-e^{\frac{(\lambda-1) x-(\lambda+1) \xi}{\epsilon \sqrt{\tau}}} d \xi \\
& =\frac{\left[e^{\frac{(\lambda-1) x-(\lambda+1) \xi}{\epsilon \sqrt{\tau}}}\right]_{\xi=0}^{\xi=x}}{-\frac{\lambda+1}{\epsilon \sqrt{\tau}}}+\frac{\left[e^{\frac{(\lambda-1) x+(1-\lambda) \xi}{\epsilon \sqrt{\tau}}}\right]_{\xi=0}^{\xi=x}}{-\frac{\lambda-1}{\epsilon \sqrt{\tau}}}+\frac{\left[e^{\frac{(\lambda+1)(x-\xi)}{\epsilon \sqrt{\tau}}}\right]_{\xi=x}^{\xi=+\infty}}{-\frac{\lambda+1}{\epsilon \sqrt{\tau}}}-\frac{\left[e^{\frac{(\lambda-1) x-(\lambda+1) \xi}{\epsilon \sqrt{\tau}}}\right]_{\xi=x}^{\xi=+\infty}}{-\frac{\lambda+1}{\epsilon \sqrt{\tau}}} \\
& =\frac{e^{-\frac{2 x}{\epsilon \sqrt{\tau}}}-e^{\frac{(\lambda-1) x}{\epsilon \sqrt{\tau}}}}{-\frac{\lambda+1}{\epsilon \sqrt{\tau}}}+\frac{1-e^{\frac{(\lambda-1) x}{\epsilon \sqrt{\tau}}}}{-\frac{\lambda-1}{\epsilon \sqrt{\tau}}}+\frac{0-1}{-\frac{\lambda+1}{\epsilon \sqrt{\tau}}}-\frac{0-e^{-\frac{2 x}{\epsilon \sqrt{\tau}}}}{-\frac{\lambda+1}{\epsilon \sqrt{\tau}}} \\
& =\frac{2 e^{-\frac{2 x}{\epsilon \sqrt{\tau}}}-e^{\frac{(\lambda-1) x}{\epsilon \sqrt{\tau}}}-1}{-\frac{\lambda+1}{\epsilon \sqrt{\tau}}}+\frac{1-e^{\frac{(\lambda-1) x}{\epsilon \sqrt{\tau}}}}{-\frac{\lambda-1}{\epsilon \sqrt{\tau}}} \\
& =\frac{\epsilon \sqrt{\tau}}{\lambda^{2}-1}\left(-2+2 \lambda e^{\frac{(\lambda-1) x}{\epsilon \sqrt{\tau}}}-2(\lambda-1) e^{-\frac{2 x}{\epsilon \sqrt{\tau}}}\right) \\
& \leq \frac{2 \epsilon \sqrt{\tau}}{1-\lambda^{2}}
\end{aligned}
$$

$\square$ 
Proof to lemma 2.3 .3 (iii).

$$
\begin{aligned}
& \int_{0}^{+\infty}\left|e^{-\frac{x+\xi}{\epsilon \sqrt{\tau}}}+\operatorname{sgn}(x-\xi) e^{-\frac{|x-\xi|}{\epsilon \sqrt{\tau}}}\right| e^{\frac{\lambda x-\xi}{\epsilon \sqrt{\tau}}} d \xi \\
= & \int_{0}^{x}\left|e^{-\frac{x+\xi}{\epsilon \sqrt{\tau}}}+e^{-\frac{x-\xi}{\epsilon \sqrt{\tau}}}\right| e^{\frac{\lambda x-\xi}{\epsilon \sqrt{\tau}}} d \xi+\int_{x}^{+\infty}\left|e^{-\frac{x+\xi}{\epsilon \sqrt{\tau}}}-e^{-\frac{\xi-x}{\epsilon \sqrt{\tau}}}\right| e^{\frac{\lambda x-\xi}{\epsilon \sqrt{\tau}}} d \xi \\
= & \frac{\left[e^{\frac{(\lambda-1) x-2 \xi}{\epsilon \sqrt{\tau}}}\right]_{\xi=0}^{\xi=x}}{\frac{-2}{\epsilon \sqrt{\tau}}}+x e^{\frac{(\lambda-1) x}{\epsilon \sqrt{\tau}}}+\frac{\left[e^{\frac{(\lambda+1) x-2 \xi}{\epsilon \sqrt{\tau}}}\right]_{\xi=x}^{\xi=+\infty}}{\frac{-2}{\epsilon \sqrt{\tau}}}-\frac{\left[e^{\frac{(\lambda-1) x-2 \xi}{\epsilon \sqrt{\tau}}}\right]_{\xi=x}^{\xi=+\infty}}{\frac{-2}{\epsilon \sqrt{\tau}}} \\
= & \frac{e^{\frac{(\lambda-3 x}{\epsilon \sqrt{\tau}}}-e^{\frac{(\lambda-1) x}{\epsilon \sqrt{\tau}}}}{\frac{-2}{\epsilon \sqrt{\tau}}}+x e^{\frac{(\lambda-1) x}{\epsilon \sqrt{\tau}}}+\frac{0-e^{\frac{(\lambda-1) x}{\epsilon \sqrt{\tau}}}}{\frac{-2}{\epsilon \sqrt{\tau}}}-\frac{0-e^{\frac{(\lambda-3) x}{\epsilon \sqrt{\tau}}}}{\frac{-2}{\epsilon \sqrt{\tau}}} \\
= & \frac{2 e^{\frac{(\lambda-3) x}{\epsilon \sqrt{\tau}}}-2 e^{\frac{(\lambda-1) x}{\epsilon \sqrt{\tau}}}}{\frac{-2}{\epsilon \sqrt{\tau}}}+x e^{\frac{(\lambda-1) x}{\epsilon \sqrt{\tau}}}
\end{aligned}
$$

In the proof of Lemma 2.3.2 (iii), we have shown that

$$
x e^{\frac{(\lambda-1) x}{\epsilon \sqrt{\tau}}} \leq \frac{\epsilon \sqrt{\tau}}{e(1-\lambda)}
$$

Therefore,

$$
\int_{0}^{+\infty}\left|e^{-\frac{x+\xi}{\sqrt{\tau}}}+\operatorname{sgn}(x-\xi) e^{-\frac{|x-\xi|}{\epsilon \sqrt{\tau}}}\right| e^{\frac{\lambda x-\xi}{\epsilon \sqrt{\tau}}} d \xi \leq \epsilon \sqrt{\tau}+\frac{\epsilon \sqrt{\tau}}{e(1-\lambda)}
$$

$\square$

Proof to lemma 2.3.3 (iii). Based on the assumption on $u_{0}$ in 2.30)

$$
\begin{aligned}
& \int_{0}^{+\infty}\left|e^{-\frac{x+\xi}{\epsilon \sqrt{\tau}}}+\operatorname{sgn}(x-\xi) e^{-\frac{|x-\xi|}{\epsilon \sqrt{\tau}}}\right| e^{\frac{\lambda x}{\epsilon \sqrt{\tau}}}\left|u_{0}(\xi)\right| d \xi \\
\leq & C_{u} e^{\frac{\lambda x}{\epsilon \sqrt{\tau}}} \int_{0}^{L_{0}}\left|e^{-\frac{x+\xi}{\sqrt{\tau}}}+\operatorname{sgn}(x-\xi) e^{-\frac{\mid x-\xi \xi}{\sqrt{\tau}}}\right| d \xi \\
= & C_{u} y_{3}(x)
\end{aligned}
$$

Calculating $y_{3}(x)$ with the assumption that $\lambda \in(0,1)$, we get 
- case (a): $x \in\left[0, L_{0}\right]$

$$
\begin{aligned}
y_{3}(x) & =\int_{0}^{x}\left(e^{-\frac{x+\xi}{\epsilon \sqrt{\tau}}}+e^{-\frac{x-\xi}{\epsilon \sqrt{\tau}}}\right) e^{\frac{\lambda x}{\epsilon \sqrt{\tau}}} d \xi+\int_{x}^{L_{0}}\left(e^{-\frac{\xi-x}{\epsilon \sqrt{\tau}}}-e^{-\frac{x+\xi}{\epsilon \sqrt{\tau}}}\right) e^{\frac{\lambda x}{\epsilon \sqrt{\tau}}} d \xi \\
& \leq \int_{0}^{x}\left(e^{-\frac{x+\xi}{\epsilon \sqrt{\tau}}}+e^{-\frac{x-\xi}{\epsilon \sqrt{\tau}}}\right) e^{\frac{\lambda x}{\epsilon \sqrt{\tau}}} d \xi+\int_{x}^{L_{0}} e^{-\frac{\xi-x}{\epsilon \sqrt{\tau}}} e^{\frac{\lambda x}{\epsilon \sqrt{\tau}}} d \xi \\
& =-\epsilon \sqrt{\tau}\left[e^{\frac{(\lambda-1) x-\xi}{\epsilon \sqrt{\tau}}}\right]_{\xi=0}^{\xi=x}+\epsilon \sqrt{\tau}\left[e^{\frac{(\lambda-1) x+\xi}{\epsilon \sqrt{\tau}}}\right]_{\xi=0}^{\xi=x}-\epsilon \sqrt{\tau}\left[e^{\frac{(\lambda+1) x-\xi}{\epsilon \sqrt{\tau}}}\right]_{\xi=x}^{\xi=L_{0}} \\
& =\epsilon \sqrt{\tau}\left(e^{\frac{(\lambda-1) x}{\epsilon \sqrt{\tau}}}-e^{\frac{(\lambda-2) x}{\epsilon \sqrt{\tau}}}+e^{\frac{\lambda x}{\epsilon \sqrt{\tau}}}-e^{\frac{(\lambda-1) x}{\epsilon \sqrt{\tau}}}+e^{\frac{\lambda x}{\epsilon \sqrt{\tau}}}-e^{\frac{(\lambda+1) x-L_{0}}{\epsilon \sqrt{\tau}}}\right) \\
& \leq 2 \epsilon \sqrt{\tau} e^{\frac{\lambda x}{\epsilon \sqrt{\tau}}} \\
& \leq 2 \epsilon \sqrt{\tau} e^{\frac{\lambda L_{0}}{\epsilon \sqrt{\tau}}}
\end{aligned}
$$

- case (b): $x \in\left[L_{0},+\infty\right]$

$$
\begin{aligned}
y_{3}(x) & =\int_{0}^{L_{0}}\left(e^{-\frac{x+\xi}{\epsilon \sqrt{\tau}}}+e^{-\frac{x-\xi}{\epsilon \sqrt{\tau}}}\right) e^{\frac{\lambda x}{\epsilon \sqrt{\tau}}} d \xi \\
& =-\epsilon \sqrt{\tau}\left[e^{\frac{(\lambda-1) x-\xi}{\epsilon \sqrt{\tau}}}\right]_{\xi=0}^{\xi=L_{0}}+\epsilon \sqrt{\tau}\left[e^{\frac{(\lambda-1) x+\xi}{\epsilon \sqrt{\tau}}}\right]_{\xi=0}^{\xi=L_{0}} \\
& =\epsilon \sqrt{\tau}\left(e^{\frac{(\lambda-1) x}{\epsilon \sqrt{\tau}}}-e^{\frac{(\lambda-1) x-L_{0}}{\epsilon \sqrt{\tau}}}+e^{\frac{(\lambda-1) x+L_{0}}{\epsilon \sqrt{\tau}}}-e^{\frac{(\lambda-1) x}{\epsilon \sqrt{\tau}}}\right) \\
& \leq \epsilon \sqrt{\tau} e^{\frac{(\lambda-1) x+L_{0}}{\epsilon \sqrt{\tau}}}
\end{aligned}
$$

Since $0<\lambda<1$,

$$
\begin{aligned}
y_{3}(x) & \leq \epsilon \sqrt{\tau} e^{\frac{\lambda L_{0}}{\sqrt{\sqrt{\tau}}}} \\
& \leq 2 \epsilon \sqrt{\tau} e^{\frac{\lambda L_{0}}{\epsilon \sqrt{\tau}}}
\end{aligned}
$$

Therefore, we get the desired inequality

$$
\int_{0}^{+\infty}\left|e^{-\frac{x+\xi}{\epsilon \sqrt{\tau}}}+\operatorname{sgn}(x-\xi) e^{-\frac{|x-\xi|}{\epsilon \sqrt{\tau}}}\right| e^{\frac{\lambda x}{\epsilon \sqrt{\tau}}}\left|u_{0}(\xi)\right| d \xi \leq 2 C_{u} \epsilon \sqrt{\tau} e^{\frac{\lambda L_{0}}{\epsilon \sqrt{\tau}}}
$$




\section{A.4 Proof to lemma 2.3.4}

Proof to lemma 2.3.4 (i).

$$
\begin{aligned}
\left|\phi_{1}(x)-e^{-\frac{x}{\epsilon \sqrt{\tau}}}\right| & =\left|\frac{e^{\frac{L-x}{\epsilon \sqrt{\tau}}}-e^{\frac{-L+x}{\epsilon \sqrt{\tau}}}}{e^{\frac{L}{\epsilon \sqrt{\tau}}}-e^{-\frac{L}{\epsilon \sqrt{\tau}}}}-e^{-\frac{x}{\epsilon \sqrt{\tau}}}\right| \\
& =\frac{e^{\frac{L-x}{\epsilon \sqrt{\tau}}}-e^{\frac{-L+x}{\epsilon \sqrt{\tau}}}-e^{\frac{L-x}{\epsilon \sqrt{\tau}}}+e^{-\frac{L+x}{\epsilon \sqrt{\tau}}}}{e^{\frac{L}{\epsilon \sqrt{\tau}}}-e^{-\frac{L}{\epsilon \sqrt{\tau}}}} \\
& =e^{-\frac{L}{\epsilon \sqrt{\tau}}}\left|\frac{e^{-\frac{x}{\epsilon \sqrt{\tau}}}-e^{\frac{x}{\epsilon \sqrt{\tau}}}}{e^{\frac{L}{\epsilon \sqrt{\tau}}}-e^{-\frac{L}{\epsilon \sqrt{\tau}}}}\right| \\
& \leq e^{-\frac{L}{\epsilon \sqrt{\tau}}}\left|\phi_{2}(x)\right|
\end{aligned}
$$

Proof to lemma 2.3.4 (ii).

$$
\phi_{2}(x)=\frac{e^{\frac{x}{\epsilon \sqrt{\tau}}}-e^{-\frac{x}{\epsilon \sqrt{\tau}}}}{e^{\frac{L}{\epsilon \sqrt{\tau}}}-e^{-\frac{L}{\epsilon \sqrt{\tau}}}}
$$

gives that

$$
\phi_{2}^{\prime}(x)=\frac{1}{\epsilon \sqrt{\tau}} \frac{e^{\frac{x}{\epsilon \sqrt{\tau}}}+e^{-\frac{x}{\epsilon \sqrt{\tau}}}}{e^{\frac{L}{\epsilon \sqrt{\tau}}}-e^{-\frac{L}{\epsilon \sqrt{\tau}}}}>0
$$

and hence

$$
\phi_{2}(x) \leq \phi_{2}(L)=1 \quad \text { for } x \in[0, L]
$$

Proof to lemma 2.3.4 (iii).

$$
\phi_{2}^{\prime}(x)=\frac{1}{\epsilon \sqrt{\tau}} \frac{e^{\frac{x}{\epsilon \sqrt{\tau}}}+e^{-\frac{x}{\epsilon \sqrt{\tau}}}}{e^{\frac{L}{\epsilon \sqrt{\tau}}}-e^{-\frac{L}{\epsilon \sqrt{\tau}}}}
$$


gives that

$$
\phi_{2}^{\prime \prime}(x)=\frac{1}{\epsilon^{2} \tau} \phi_{2}(x)>0
$$

and hence

$$
\begin{aligned}
\phi_{2}^{\prime}(x) & \leq \phi_{2}^{\prime}(L) \\
& =\frac{1}{\epsilon \sqrt{\tau}} \frac{e^{\frac{L}{\epsilon \sqrt{\tau}}}+e^{-\frac{L}{\epsilon \sqrt{\tau}}}}{e^{\frac{L}{\epsilon \tau}}-e^{-\frac{L}{\epsilon \sqrt{\tau}}}} \\
& =\frac{1}{\epsilon \sqrt{\tau}} \frac{e^{\frac{2 L}{\epsilon \sqrt{\tau}}}+1}{e^{\frac{2 L}{\epsilon \sqrt{\tau}}}-1} \\
& \leq \frac{2}{\epsilon \sqrt{\tau}}
\end{aligned}
$$

if $\epsilon \ll 1$ for $x \in[0, L] . \square$ 


\section{BIBLIOGRAPHY}

[1] J. L. Bona, H. Chen, S. M. Sun, and B.-Y. Zhang. Comparison of quarter-plane and two-point boundary value problems: the BBM-equation. Discrete Contin. Dyn. Syst., 13(4):921-940, 2005.

[2] J. L. Bona and L. Luo. Initial-boundary value problems for model equations for the propagation of long waves. In Evolution equations (Baton Rouge, LA, 1992), volume 168 of Lecture Notes in Pure and Appl. Math., pages 63, 65-94. Dekker, New York, 1995.

[3] S. Buckley and M. Leverett. Mechanism of fluid displacement in sands. Petroleum Transactions, AIME, 146:107-116, 1942.

[4] B. Cockburn, C. Johnson, C.-W. Shu, and E. Tadmor. Advanced numerical approximation of nonlinear hyperbolic equations, volume 1697 of Lecture Notes in Mathematics. Springer-Verlag, Berlin, 1998. Papers from the C.I.M.E. Summer School held in Cetraro, June 23-28, 1997, Edited by Alfio Quarteroni, Fondazione C.I.M.E.. [C.I.M.E. Foundation].

[5] B. Cockburn, G. E. Karniadakis, and C.-W. E. Shu. Discontinuous Galerkin Methods: Theory, Computation and Applications. Lecture Notes in Computational Science and Engineering. 2000.

[6] A. Corey. The interrelation between gas and oil relative permeabilities. Producer's Monthly, 19(1):38-41, 1954.

[7] C. Cuesta, C. J. van Duijn, and J. Hulshof. Infiltration in porous media with dynamic capillary pressure: travelling waves. European J. Appl. Math., 11(4):381397, 2000.

[8] D. A. DiCarlo. Experimental measurements of saturation overshoot on infiltration. Water Resources Research, 40:4215.1 - 4215.9, Apr. 2004.

[9] S. Hassanizadeh and W. Gray. Mechanics and thermodynamics of multiphase flow in porous media including interphase boundaries. Adv. Water Resour., 13:169-186, 1990. 
[10] S. Hassanizadeh and W. Gray. Thermodynamic basis of capillary pressure in porous media. Water Resour. Res., 29:3389-3405, 1993.

[11] H. Hugoniot. Propagation des Mouvements dans les Corps et specialement dans les Gaz Parfaits (in French). Journal de l'Ecole Polytechnique, 57:3-97, 1987.

[12] A. Kurganov and D. Levy. A third-order semidiscrete central scheme for conservation laws and convection-diffusion equations. SIAM J. Sci. Comput., 22(4):1461-1488 (electronic), 2000.

[13] A. Kurganov and E. Tadmor. New high-resolution central schemes for nonlinear conservation laws and convection-diffusion equations. J. Comput. Phys., 160(1):241-282, 2000.

[14] R. J. LeVeque. Numerical methods for conservation laws. Lectures in Mathematics ETH Zürich. Birkhäuser Verlag, Basel, second edition, 1992.

[15] R. J. LeVeque. Finite volume methods for hyperbolic problems. Cambridge Texts in Applied Mathematics. Cambridge University Press, Cambridge, 2002.

[16] W. J. Macquorn Rankine. On the thermodynamic theory of waves of finite longitudinal disturbance. Royal Society of London Philosophical Transactions Series I, 160:277-288, 1870.

[17] H. Nessyahu and E. Tadmor. Nonoscillatory central differencing for hyperbolic conservation laws. J. Comput. Phys., 87(2):408-463, 1990.

[18] O. A. Oleŭnik. Discontinuous solutions of non-linear differential equations. Uspehi Mat. Nauk (N.S.), 12(3(75)):3-73, 1957.

[19] C. J. Van Duijn, A. Mikelic, and I. Pop. Effective Buckley-Leverett equations by homogenization. Progress in industrial mathematics at ECMI, pages $42-52$, 2000 .

[20] C. J. Van Duijn, A. Mikelic, and I. S. Pop. Effective equations for two-phase flow with trapping on the micro scale. SIAM Journal on Applied Mathematics, 62(5):1531-1568, 2002.

[21] C. J. van Duijn, L. A. Peletier, and I. S. Pop. A new class of entropy solutions of the Buckley-Leverett equation. SIAM J. Math. Anal., 39(2):507-536 (electronic), 2007. 
[22] Y. Xu and C.-W. Shu. A local discontinuous Galerkin method for the CamassaHolm equation. SIAM J. Numer. Anal., 46(4):1998-2021, 2008.

[23] Y. Xu and C.-W. Shu. Local discontinuous Galerkin method for the HunterSaxton equation and its zero-viscosity and zero-dispersion limits. SIAM J. Sci. Comput., 31(2):1249-1268, 2008/09. 